\title{
Spacelike and timelike pion electromagnetic form factor and Fock state components within the light-front dynamics
}

\author{
J.P. B. C. de Melo, ${ }^{1}$ T. Frederico, ${ }^{2}$ E. Pace, ${ }^{3}$ and G. Salmè ${ }^{4}$ \\ ${ }^{1}$ Centro de Ciências Exatas e Tecnológicas, Universidade Cruzeiro do Sul, 08060-070, and Instituto de Física Teórica, \\ Universidade Estadual Paulista, 01405-900, São Paulo, Brazil \\ ${ }^{2}$ Departamento de Física, Instituto Tecnológico da Aeronáutica, Centro Técnico Aeroespacial, 12.228-900 São José dos Campos, \\ São Paulo, Brazil \\ ${ }^{3}$ Dipartimento di Fisica, Università di Roma "Tor Vergata" and Istituto Nazionale di Fisica Nucleare, Sezione Tor Vergata, \\ Via della Ricerca Scientifica 1, I-00133 Roma, Italy \\ ${ }^{4}$ Istituto Nazionale di Fisica Nucleare, Sezione Roma I, Piazzale A. Moro 2, I-00185 Roma, Italy \\ (Received 29 July 2005; revised manuscript received 7 March 2006; published 13 April 2006)
}

\begin{abstract}
The simultaneous investigation of the pion electromagnetic form factor in the space- and timelike regions within a light-front model allows one to address the issue of nonvalence components of the pion and photon wave functions. Our relativistic approach is based on a microscopic vector-meson-dominance model for the dressed vertex where a photon decays in a quark-antiquark pair, and on a simple parametrization for the emission or absorption of a pion by a quark. The results show an excellent agreement in the space like region up to $-10(\mathrm{GeV} / c)^{2}$, while in timelike region the model produces reasonable results up to $10(\mathrm{GeV} / c)^{2}$.
\end{abstract}

DOI: 10.1103/PhysRevD.73.074013

\section{INTRODUCTION}

Electroweak properties are widely used as an important source of information on the structure of hadrons. In particular within the framework of light-front (LF) dynamics [1-5], a large number of papers have been devoted to the study of nuclei and hadrons (see, e.g., [6-22], just to give a partial account of previous works with a finite number of constituents).

The LF dynamics allows one to exploit the intuitive language of the Fock space. Indeed the Fock-space language is particularly meaningful within LF dynamics, since: "The simplicity of the light-cone Fock representation as compared to that in equal-time quantization is directly linked to the fact that the physical vacuum state has a much simpler structure on the light cone because the Fock vacuum is an exact eigenstate of the full Hamiltonian" [2]. In LF dynamics for a fermionic system, vacuum phenomena, such as spontaneous chiral symmetry breaking, have their counterpart in the physics of the zero modes (see, e.g. [2,23-25]).

Another basic motivation for choosing the LF dynamics is represented by the striking feature that the Fock decomposition is stable under LF boosts, since they are of kinematical nature and therefore do not change the number of particles, i.e., are diagonal in the Fock space.

Therefore the LF dynamics is a suitable framework for the investigation of the Fock expansion for mesons and baryons, viz

$$
\begin{aligned}
\mid \text { meson }\rangle & =|q \bar{q}\rangle+|q \bar{q} q \bar{q}\rangle+|q \bar{q} g\rangle+\ldots \\
\mid \text { baryon }\rangle & =|q q q\rangle+|q q q q \bar{q}\rangle+|q q q g\rangle+\ldots
\end{aligned}
$$

In particular, within the LF dynamics the electromagnetic form factor of the pion has been the object of many
PACS numbers: 12.39.Ki, 12.40.Vv, 13.40.Gp, 14.40.Aq

papers (see, e.g., Refs. [7,9-12,14,17,20,21]). Indeed the pion electromagnetic form factor yields a simple tool for the investigation of pion and photon microscopic structure in terms of hadronic constituents. To our knowledge, however, there is no paper where both the spacelike and the timelike pion form factor are evaluated within the same microscopical model, taking into account the fermionic nature of the constituent quarks. Previously, in the timelike region only the form factor for a pion composed by boson constituents was explored in the light-front quantization [17]. Such an investigation was performed by using both a direct calculation and an analytic continuation, in a Drell-Yan-West reference frame, with pointlike vertices. Because of the pointlike nature of the photon-quark vertex, such a model could not exploit the rich structure of the meson excited states and only a $\rho$-meson type peak was recovered.

In what follows we will present an approach to investigate in a common framework the pion and photon vertex functions, with the perspective of an extension of our approach to the nucleon. The intuitive language of the Fock space will be adopted to analyze the above mentioned vertex functions.

The aim of this work is to give a unified, microscopical evaluation of the electromagnetic form factor of the pion, both in the spacelike (SL) and in the timelike (TL) regions. Peculiar features of our approach are the pion and photon dressed vertex functions, both in the valence and in the nonvalence sectors, as well as a careful consideration of the fermionic nature of the constituents. A first presentation of our approach was given in Ref. [26].

The choice of the reference frame where the form factor calculation is carried out has a fundamental role, as shown in previous works in the spacelike region $[14,19,21,27]$ and 
in the timelike one [17]. For a unified, direct calculation of the pion form factor in TL and SL regions, a reference frame is needed where the plus component of the momentum transfer, $q^{+}=q^{0}+q^{3}$, is different from zero (otherwise, $q^{2}=q^{+} q^{-}-q_{\perp}^{2}$ cannot be positive). As a matter of fact, a reference frame where $q^{+} \neq 0$ allows one to analyze, in a common framework [26], the pair-production process (Z-diagram contribution) [18], i.e. the effect of multiquark propagation, as well as the ultrarelativistic effect of the so-called instantaneous contributions and the hadronic components of the photon wave function $[2,28]$.

In Ref. [29] it was shown that, within the Hamiltonian LF dynamics (HLFD), a Poincaré covariant and conserved current operator can be obtained from the matrix elements of the free current, evaluated in the Breit reference frame, where the initial and the final total momenta of the system are directed along the spin quantization axis $z$. Following Ref. [29], we calculate the pion form factor in a reference frame where $\mathbf{q}_{\perp}=0$ and $q^{+}>0$.

Different roads are possible to construct a relativistic description for the electromagnetic form factor of the pion. One road is represented by the HLFD, where a fixed number of constituents is retained and an Ansatz for the 3D pion wave function is introduced. Unfortunately, within such an approach to describe the timelike form factor one has to use an analytic continuation, plagued by well-known limits (tiny variations in the spacelike analytic form that interpolates the model results produce largely different results in the timelike region). A second road is represented by a fully covariant approach, e.g. in the manner of Mandelstam [30]. However, up to now, there is no full solution, $\Lambda(k, P)$, of the four-dimensional Bethe-Salpeter equation for an interacting system composed by fermions, both on the mass shell and off the mass shell of the system. Therefore Ansätze for the 4D vertex functions relevant for the problem under consideration and with a simple analytic structure are adopted (e.g., as was done in Refs. [19,21]). Unfortunately, those Ansätze can only partially retain the full analytic complexity of the true vertex functions (given by the dynamics governing the system under consideration). A third road, the one we are going to explore, is represented by an approach where Ansätze for the 3D light-front amplitudes, present in the Fock expansion of a hadron state, are assumed, which embed as much as possible the successful phenomenology developed within the constituent quark model (CQM), i.e. retain the ability to describe the meson spectra. In particular, in our approach, (i) the light-front amplitude for the valence component of the meson states will be put in relation with 3D HLFD wave functions, (ii) the light-front amplitude for the $|2 q 2 \bar{q}\rangle$ is approximated according to Ref. [18], while (iii) all the other components are put equal to zero. This amounts to truncating the Fock expansion to the lowest states. This approximation preserves kinematical boost invariance, but, as is well known, not the other symmetries. As it occurs for the other roads, the comparison with the experimental data will give the extent to which the proposed approach is a reasonable one.

To generate the diagrams that the developed phenomenology has shown important for the evaluation of the pion form factor, we perform an approximate integration on the LF energy, $k^{-}=k^{0}-k^{3}$, of the Mandelstam formula [30] with a dressed photon vertex, disregarding the contribution of the singularities of the hadron Bethe-Salpeter amplitudes. As shown in the literature [18,19,31], the LF energy integration allows one to obtain an explicit correspondence between the sum of LF time-ordered amplitudes, including valence and nonvalence diagrams, and the original covariant amplitude. In this work this procedure is used as an effective tool for obtaining regularized expressions for the different contributions to the form factor in an arbitrary reference frame (it is worth noting that we are not using the standard $q^{+}=0$ reference frame to evaluate the pion form factor). Let us note that the $k^{-}$integration is able to immediately produce the familiar kinematical constraints on $k^{+}$of the valence and nonvalence contributions.

In field theory the state has an infinite number of components in the Fock space. In principle, the infinite set of coupled eigenvalue equation for the Hamiltonian operator in the Fock space can be replaced by an effective squared mass operator acting in the valence sector [2]. We will identify this effective mass operator with the mass operator used in HLFD and then the hadron vertex functions in the valence region will be connected (up to a normalization factor) to the LF wave function of the valence component of the hadron state. This is an essential step in our approach, since it allows us to exploit the successful phenomenology developed within the CQM in order to establish for the first time a microscopical connection between the pion form factor and the meson spectrum through the Hamiltonian LF dynamics. Furthermore, the concept of hadronic valence, i.e. $q \bar{q}$, component of the photon wave function will be introduced $[2,28]$.

The main difficulties to be dealt with are (i) how to construct the photon-hadron coupling when a $q \bar{q}$ pair is produced by a photon with $q^{+}>0$; and (ii) how to describe the nonvalence content relevant for the process under consideration, both in the pion and in the photon wave functions.

The first issue is addressed by using a covariant generalization of the vector-meson-dominance (VMD) approach (see, e.g., [32]) at the level of the photon vertex function (see Ref. [26]) written in terms of VM vertex functions. These latter vertex functions will be related in the valence region to the corresponding LF wave functions. As a matter of fact, it is necessary to construct the Green's function of the interacting $q \bar{q}$ pair in the $1^{-}$channel. For the description of the vector-meson vertex functions in the valence sector we use the eigenfunctions of the square mass operator proposed in Refs. [33,34]. The simplified version of 
the model that we are going to use [35] includes confinement through a harmonic oscillator potential. The model showed a universal and satisfactory description of the experimental values of the masses of both singlet and triplet $S$-wave mesons and the corresponding radial excitations [35], giving a natural explanation of the almost linear relationship between the mass squared of excited states and the radial quantum number $n[36,37]$. Therefore such a relativistic model, that retains the main feature of the spectra and at the same time allows one to perform simple numerical calculations, will be adopted for both pseudoscalar and vector mesons.

The second issue, i.e. the contribution of the nonvalence $(2 q 2 \bar{q})$ components of the pion and photon wave functions, is addressed using a model where a quark in the valence component radiates a pair by a contact interaction [18]. This interaction is described through a pseudoscalar coupling of quark and pion fields, multiplied by a constant. In a recent study of meson decay processes within LF dynamics [18], this approximation was shown to give a good description of the experimental data. Here, we just follow the above suggestion to parameterize the radiative pion emission amplitude from the quark.

Another important point to be carefully treated is the contribution of the instantaneous terms, which is strictly related to the fermionic nature of the constituents. We remind the reader that the Dirac propagator can be decomposed using the light-front momentum components [2], as follows:

$$
\frac{k+m}{k^{2}-m^{2}+\imath \epsilon}=\frac{k_{\mathrm{on}}+m}{k^{+}\left(k^{-}-k_{\mathrm{on}}^{-}+\frac{\imath \epsilon}{k^{+}}\right)}+\frac{\gamma^{+}}{2 k^{+}},
$$

where $\gamma^{+}=\gamma^{0}+\gamma^{3}$ and $k_{\text {on }}^{-}=\left(\left|\mathbf{k}_{\perp}\right|^{2}+m^{2}\right) / k^{+}$. The second term on the right-hand side of Eq. (2) is an instantaneous term in the light-front time, related to the so-called zero modes. As already known (see, e.g., [21]), the instantaneous contributions play a dominant role in the description of the pion electromagnetic form factor in the spacelike region, in a reference frame where $q^{+}>0$. Therefore a special care is devoted in the present work to the treatment of the instantaneous contributions in the light-cone representation of the fermion propagators. In particular the contributions of the zero modes are under control, thanks to the suitable momentum behavior of the hadron vertex functions. It should be pointed out that the effects of the instantaneous terms is emphasized by the small mass of the pion.

Our description contains a small set of parameters: the oscillator strength, the constituent quark mass, and the width for the vector mesons. We use experimental widths for the vector-mesons, when available [38], while for the unknown widths of the radial excitations we use a single width as a fitting parameter. The constant involved in the description of the nonvalence component can be fixed by the pion charge normalization in the limit of a vanishing pion mass. To evaluate the instantaneous vertex functions we introduce a simple Ansatz with only one parameter, instead of a more sophisticated treatment left for further investigations (see Sec. X). It should be pointed out that current analyses of the experimental data in the TL region are carried on by using large sets of parameters (see e.g. $[39,40])$

In the present paper in order to simplify the numerical calculations, we use a massless pion. It is worth noting that in the timelike region the full result for the pion form factor is always given by the pair-production process ("Z-diagram") alone, independently of this approximation, while in the spacelike region only the " $Z$-diagram" contribution $[14,19,21,22,27,41]$ survives for a massless pion [26]. The importance of the " $Z$-diagram" contribution to the electromagnetic current for $q^{+}>0$ was also recently investigated in the context of the Bethe-Salpeter equation within the light-front quantization in Ref. [42].

Since in this paper we wish to establish a connection between the vertex functions in the valence region and the HLFD wave functions, we have to isolate in the triangle diagram the contributions where the vertex functions appear with both the quarks on the mass shell from the instantaneous terms and from the terms with vertex functions outside the valence-sector range, which represent the absorption or the emission of a pion by a quark. Given the complexity of the above mentioned aim, the actual calculations will be performed using simple assumptions for the different vertex functions that are needed. We intend to explore more elaborate approximations for the vertex functions in future works.

This work is organized as follows. In Sec. II, we present the general form of the covariant electromagnetic form factor of the pion in impulse approximation and our vector-meson-dominance approach for the dressed photon vertex. In Sec. III, we first decompose the triangle diagram in on-shell and instantaneous contributions. Then we integrate for $q^{+}>0$ on the light-front energy in the momentum loop of the triangle diagram, under analytical assumptions for the vertex functions.

In order to construct a bridge with the Hamiltonian language, the valence components of the light-front meson and photon wave functions are defined in Sec. IV. In Sec. V, we discuss the contribution of the nonvalence component of the photon to the timelike current, which appears through the vertex for the radiative emission of pions by a virtual quark inside the photon. In this section, we also discuss the contribution of the nonvalence component of the pion wave function to the spacelike current. In Sec. VI, we introduce the pion and vector-meson wave functions in the expression of the triangle diagram. The timelike and spacelike pion form factor, written in terms of the valence components of the meson wave functions and of the emission/absorption vertices, is derived in Secs. VII and VIII, respectively. In Sec. IX, we briefly revise the light-front 
model for the pion and vector mesons and conclude the derivation of our model for the pion form factor with a discussion on our treatment of the vertex functions for the instantaneous terms. In Sec. X, we compare our numerical results for the pion form factor with the experimental data in the momentum transfer range between $-10(\mathrm{GeV} / c)^{2}$ and $+10(\mathrm{GeV} / c)^{2}$. In Sec. XI, our conclusions are presented.

\section{COVARIANT EM FORM FACTOR OF THE PION}

As mentioned in Sec. I, we wish to generate the expression of the pion form factor within LF dynamics in a general reference frame (remind that for a unified evaluation of the pion form factor, both in the SL and in the TL region, we are going to use a frame where $q^{+}>0$ ). As an effective tool to this end, we integrate over the LF energy $k^{-}$(see the next section) the covariant expression, evaluated in impulse approximation [30], for the amplitude of the processes $\pi \gamma^{*} \rightarrow \pi^{\prime}$, or $\gamma^{*} \rightarrow \pi \pi^{\prime}$, where the meson $\pi^{\prime}$ is a pion in the elastic case or an antipion in the production process (see the triangle diagram of Fig. 1). In the timelike region this covariant expression reads (see Fig. 2)

$$
\begin{aligned}
j^{\mu}= & \left\langle\bar{\pi} \pi\left|J^{\mu}(q)\right| 0\right\rangle \\
= & -\imath 2 e \frac{m^{2}}{f_{\pi}^{2}} N_{c} \int \frac{d^{4} k}{(2 \pi)^{4}} \Lambda_{\bar{\pi}}\left(k-P_{\pi}, P_{\bar{\pi}}\right) \bar{\Lambda}_{\pi}\left(k, P_{\pi}\right) \\
& \times \operatorname{Tr}\left[S\left(k-P_{\pi}\right) \gamma^{5} S(k-q) \Gamma^{\mu}(k, q) S(k) \gamma^{5}\right],
\end{aligned}
$$

where $N_{c}=3$ is the number of colors; $S(p)=[1 /(\not p-$ $m+l \epsilon)]$ is the quark propagator with $m$ the mass of the constituent quark; $q^{\mu}$ is the virtual-photon momentum; $P_{\pi}^{\mu}$ and $P_{\bar{\pi}}^{\mu}$ are the pion momenta. The factor 2 stems from isospin algebra, since

$$
\operatorname{Tr}\left[\frac{\tau_{x}-\imath \tau_{y}}{\sqrt{2}} \frac{1+\tau_{z}}{2} \frac{\tau_{x}+{ }_{l} \tau_{y}}{\sqrt{2}}\right]=2,
$$

where $\left(1+\tau_{z}\right) / 2$ is the isospin factor of the current and the other isospin factors in Eq. (4) pertain to the pions.

The function $\bar{\Lambda}_{\pi}\left(k, P_{\pi}\right)$ is the momentum component of the $q \bar{q}$ vertex function for the outgoing pion, which must be taken as a symmetric function of the $q, \bar{q}$ momenta. In this

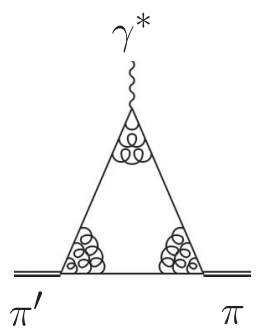

FIG. 1. Covariant amplitude for $\pi \gamma^{*} \rightarrow \pi^{\prime}$, or $\gamma^{*} \rightarrow \pi \pi^{\prime}$. The final meson $\pi^{\prime}$ is a pion in the elastic case or an antipion in the production process.

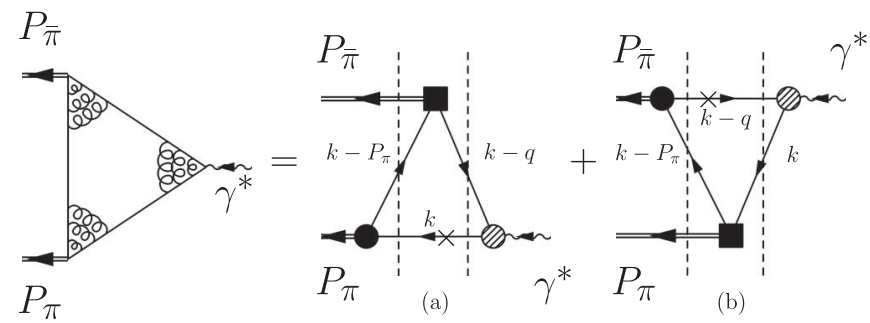

FIG. 2. Dressed photon-decay amplitude $\left(\gamma^{*} \rightarrow \pi \bar{\pi}\right)$ with two possible $x^{+}$time (light-front time) orderings, represented by diagrams (a) and (b). The two dashed vertical lines represent different light-front times (the light-front time flows from the right to the left). Diagrams (a) and diagram (b) contain the processes $q \rightarrow q \bar{\pi}$ and $q \rightarrow q \pi$, respectively, represented by a full square. The crosses indicate the quark lines which are on shell, after the $k^{-}$integration. The dashed circle represents the dressed photon vertex (see Fig. 4 for details).

vertex function, $P_{\pi}$ is the momentum of the outgoing pion and $k$ is the momentum of the incoming quark (see Fig. 2). The "bar" notation on the vertex function labels the adjoint Bethe-Salpeter amplitude, i.e., the solution of a Bethe-Salpeter equation where the two-body irreducible kernel is placed on the right of the amplitude, while for the Bethe-Salpeter amplitude it is placed on the left [43,44]. This is a well-known property of time orderings implied by the Mandelstam formula, for initial and final states [30,43]. The vertex function is defined by the following equation:

$$
\begin{aligned}
\iota & \frac{k+m}{k^{2}-m^{2}+\imath \epsilon} \gamma_{5} \Lambda_{\pi}\left(k, P_{\pi}\right) \frac{\not k^{\prime}+m}{k^{\prime 2}-m^{2}+\imath \epsilon} \delta^{4}\left(k^{\prime}+P_{\pi}-k\right) \\
= & \frac{1}{(2 \pi)^{4}} \int d^{4} x d^{4} y \exp i\left(k^{\prime} \cdot y-k \cdot x\right) \\
\quad & \times\left\langle 0|\mathrm{~T}[q(x) \bar{q}(y)]| P_{\pi}\right\rangle
\end{aligned}
$$

where $q(x)$ is the quark field.

To obtain the current matrix element for the spacelike region, $P_{\pi}^{\mu}$ should be replaced by $-P_{\pi}^{\mu}$ and $\bar{\pi}$ by $\pi^{\prime}$. Then the pion vertices $\bar{\Lambda}_{\pi}\left(k, P_{\pi}\right)$ and $\Lambda_{\bar{\pi}}\left(k-P_{\pi}, P_{\bar{\pi}}\right)$ in Eq. (3) are to be changed with $\Lambda_{\pi}\left(-k, P_{\pi}\right)$ and $\bar{\Lambda}_{\pi^{\prime}}\left(k+P_{\pi}, P_{\pi^{\prime}}\right)$, respectively (see Fig. 3). The momentum dependence of the vertex functions $\Lambda_{\bar{\pi}}\left(k-P_{\pi}, P_{\bar{\pi}}\right), \bar{\Lambda}_{\pi}\left(k, P_{\pi}\right)$ and of the photon vertex function $\Gamma^{\mu}(k, q)$ (see also [19]) is expected to regularize the integrals of Eq. (3).

The dressed photon vertex, $\Gamma^{\mu}(k, q)$, is related to the photon Bethe-Salpeter amplitude, which is defined from the three-point function in the standard form:

$$
\begin{aligned}
\imath \frac{k^{\prime}+m}{k^{2}-m^{2}+\imath \epsilon} \Gamma^{\mu}(k, q) \frac{\not k+m}{k^{2}-m^{2}+\imath \epsilon} \delta^{4}\left(k^{\prime}+q-k\right) \\
=\frac{1}{(2 \pi)^{4}} \int d^{4} x d^{4} x^{\prime} d^{4} x^{\prime \prime} \exp i\left(k^{\prime} \cdot x^{\prime}-k \cdot x+q \cdot x^{\prime \prime}\right) \\
\quad \times R_{3}^{\mu}\left(x, x^{\prime}, x^{\prime \prime}\right) .
\end{aligned}
$$

The three-point function is given by 


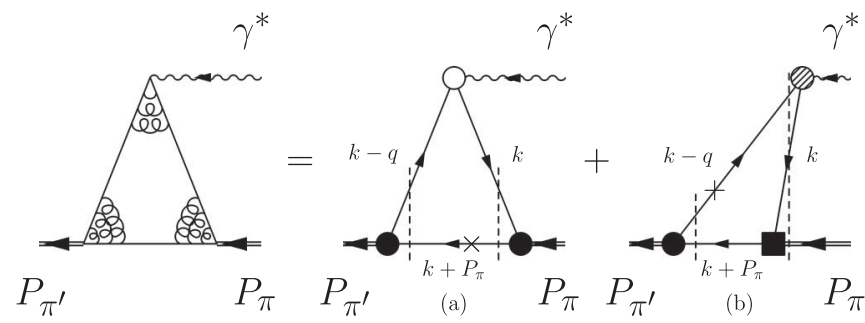

FIG. 3. Diagrammatic representation of the spacelike elastic form factor of the pion for $q^{+}>0$. The light-front time ordering allows one to single out two-quark and four-quark configurations at different light-front times, as indicated by the dashed vertical lines. Diagram (a), where $0 \leq-k^{+} \leq P_{\pi}^{+}$, represents the contribution of the valence component in the wave function of the initial pion. Diagram (b), where $0 \leq k^{+} \leq q^{+}$, represents the nonvalence contribution to the pion form factor (pair-production process). Both processes contain the contribution from the dressed photon vertex. The full square is the vertex function which describes a pion absorption by a quark. The dashed circle represents the dressed photon vertex (see Fig. 4 for details).

$$
R_{3}^{\mu}\left(x, x^{\prime}, x^{\prime \prime}\right)=\left\langle 0\left|\mathrm{~T}\left[q(x) \bar{q}\left(x^{\prime \prime}\right) \gamma^{\mu} q\left(x^{\prime \prime}\right) \bar{q}\left(x^{\prime}\right)\right]\right| 0\right\rangle,
$$

which is the matrix element between the vacuum states of the time-ordered product of the four-quark fields written above [44].

The central assumption of our paper is the microscopical description of the dressed photon vertex, $\Gamma^{\mu}(k, q)$, in the processes where a photon with $q^{+}>0$ decays in a quarkantiquark pair. In these processes we consider for the photon vertex, dressed by the interaction between the $q \bar{q}$ pair, the following covariant vector-meson-dominance approximation (see Fig. 4)

$$
\begin{aligned}
\Gamma^{\mu}(k, q)= & \sqrt{2} \sum_{n}\left[-g^{\mu \nu}+\frac{q^{\mu} q^{\nu}}{M_{n}^{2}}\right] \hat{V}_{n \nu}(k, k-q) \Lambda_{n}(k, q) \\
& \times \frac{f_{V n}}{\left[q^{2}-M_{n}^{2}+\imath M_{n} \tilde{\Gamma}_{n}\left(q^{2}\right)\right]},
\end{aligned}
$$

where

$$
\left[-g^{\mu \nu}+\frac{q^{\mu} q^{\nu}}{M_{n}^{2}}\right] \frac{1}{\left[q^{2}-M_{n}^{2}+\imath M_{n} \tilde{\Gamma}_{n}\left(q^{2}\right)\right]}
$$

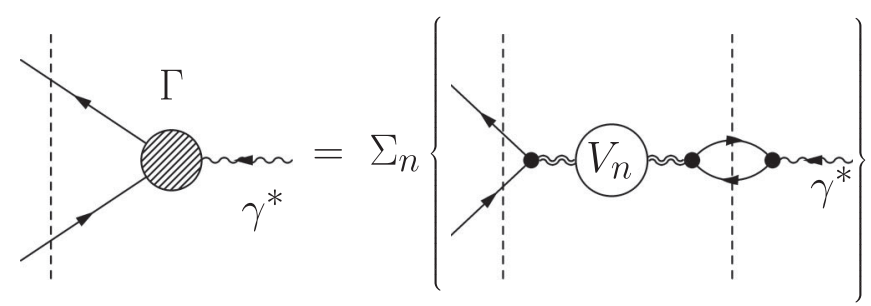

FIG. 4. Dressed photon vertex. The double-wiggly lines represent the Green function describing the propagation of the vector meson $V_{n}$. The loop on the right represents the VM decay constant, $f_{V n}$ (see Appendix A). is the vector meson propagator [45]. In Eq. (8) $f_{V n}$ is the decay constant of the $n$th vector-meson in a virtual photon, $M_{n}$ the corresponding mass, $\Lambda_{n}(k, q)$ gives the momentum dependence and $\hat{V}_{n \nu}(k, k-q)$ the Dirac structure of the VM vertex function, while $\tilde{\Gamma}_{n}\left(q^{2}\right)$ is the total decay width. Let us approximate Eq. (8) considering on-shell quantities for the VM in the numerator, i.e. let us replace $q^{-}$with $P_{n}^{-}=\left(\left|\mathbf{q}_{\perp}\right|^{2}+M_{n}^{2}\right) / q^{+}$. This approximation amounts to take the numerator in Eq. (8) for each vector-meson term at the respective pole, obtaining the correct result where the contribution is maximum. The motivation of this approximation will be clear in Sec. IV, where the on-shell VM vertex function $\left[\epsilon_{\lambda}\left(P_{n}\right) \cdot \hat{V}_{n}\left(k, k-P_{n}\right)\right] \Lambda_{n}\left(k, P_{n}\right)$ is related to the HLFD vector-meson wave function. Then the photon vertex becomes

$$
\begin{aligned}
\Gamma^{\mu}(k, q)= & \sqrt{2} \sum_{n, \lambda}\left[\epsilon_{\lambda}\left(P_{n}\right) \cdot \hat{V}_{n}\left(k, k-P_{n}\right)\right] \Lambda_{n}\left(k, P_{n}\right) \\
& \times \frac{\left[\epsilon_{\lambda}^{\mu}\left(P_{n}\right)\right]^{*} f_{V n}}{\left[q^{2}-M_{n}^{2}+\imath M_{n} \tilde{\Gamma}_{n}\left(q^{2}\right)\right]},
\end{aligned}
$$

where $\epsilon_{\lambda}\left(P_{n}\right)$ is the VM polarization. Note that the total momentum for an on-shell vector meson is $P_{n}^{\mu} \equiv\left\{P_{n}^{-}=\right.$ $\left.\left(\left|\mathbf{q}_{\perp}\right|^{2}+M_{n}^{2}\right) / q^{+}, \mathbf{P}_{n \perp}=\mathbf{q}_{\perp}, P_{n}^{+}=q^{+}\right\}, \quad$ while $q^{\mu} \equiv$ $\left\{q^{-}, \mathbf{q}_{\perp}, q^{+}\right\}$and that at the production vertex, see Fig. 4, the light-front three-momentum is conserved.

In Eq. (8) the sum runs over all the possible vector mesons. The vector-meson decay constant $f_{V n}$ can be obtained from the definition [16]

$$
\epsilon_{\lambda}^{\mu} \sqrt{2} f_{V, n}=\left\langle 0\left|\bar{q}(0) \gamma^{\mu} q(0)\right| \phi_{n, \lambda}\right\rangle
$$

with $\left|\phi_{n, \lambda}\right\rangle$ the vector-meson state. A detailed expression for $f_{V n}$ is given in Appendix A. The total decay width in the denominator of Eqs. (9) and (10), $\tilde{\Gamma}_{n}\left(q^{2}\right)$, is vanishing in the SL region. In the TL region it is assumed to be equal to

$$
\tilde{\Gamma}_{n}\left(q^{2}\right)=\Gamma_{n}\left[\frac{p\left(q^{2}\right)}{p\left(M_{n}^{2}\right)}\right]^{3}\left[\frac{M_{n}^{2}}{q^{2}}\right]^{1 / 2},
$$

where $p\left(q^{2}\right)=\left[q^{2}-4 m_{\pi}^{2}\right]^{1 / 2} / 2[46-48]$.

In Ref. [49] the following expression was used for the Dirac structure, $\hat{V}_{n}\left(k, k-P_{n}\right)$, of the vector-meson vertex:

$$
\hat{V}_{n}^{\mu}\left(k, k^{\prime}\right)=\gamma^{\mu}-\frac{M_{n}}{2} \frac{k^{\mu}+k^{\prime \mu}}{P_{n} \cdot k+m M_{n}}
$$

where $k^{\prime}=k-P_{n}$.

Let us consider, instead of Eq. (13), a symmetric form for $\hat{V}_{n}\left(k, k-P_{n}\right)$ :

$$
\begin{aligned}
\hat{V}_{n}^{\mu}\left(k, k-P_{n}\right) & =\gamma^{\mu}-M_{n} \frac{k^{\mu}+k^{\prime \mu}}{P_{n} \cdot k-P_{n} \cdot k^{\prime}+2 m M_{n}} \\
& =\gamma^{\mu}-\frac{k^{\mu}+k^{\prime \mu}}{M_{n}+2 m} .
\end{aligned}
$$

If in Eq. (14) both the CQ's are taken on their mass shell 
[i.e., $k^{-}=k_{\text {on }}^{-}=\left(\left|\mathbf{k}_{\perp}\right|^{2}+m^{2}\right) / k^{+}$] and the VM mass, $M_{n}$, is replaced by the free mass, $M_{0}$, of the quarks in a system of total momentum $q^{\mu}$,

$$
M_{0}=\left[\left(k_{\mathrm{on}}+(q-k)_{\mathrm{on}}\right) \cdot\left(k_{\mathrm{on}}+(q-k)_{\mathrm{on}}\right)\right]^{1 / 2}
$$

one obtains

$$
\left[\hat{V}_{n}^{\mu}(k, k-q)\right]_{\mathrm{on}}=\gamma^{\mu}-\frac{k_{\mathrm{on}}^{\mu}-(q-k)_{\mathrm{on}}^{\mu}}{M_{0}+2 m} .
$$

This form coincides with the on-shell expression given in Ref. [8] for the ${ }^{3} S_{1}$ vector-meson vertex, but then $\Gamma^{\mu}(k, q)$ of Eq. (10) is not anymore a four-vector.

Let us note that to obtain the pion form factor only one of the components of the current is actually needed. In the following we will derive the pion form factor from the plus component.

In Ref. [26] in order to evaluate the pion form factor we considered the plus component of Eq. (10), where $\Lambda_{n}$ and the VM polarizations were taken at the vector-meson pole, and the on-shell expression for $\hat{V}_{n}^{\mu}$, as given by Eq. (16), was used, in order to have the structure of the VM vertex suggested by the Hamiltonian LF dynamics.

In Appendix B, starting from Eq. (8), we propose a current which satisfies current conservation. In the reference frame, where $q^{+}=M_{n}>0$ and $\mathbf{q}_{\perp}=0$, the $n$th term of this current has exactly the same plus component as the $n$th term of the current defined in Eq. (10).

One might wonder that a bare $\gamma^{\mu}$ coupling term should be added to the current defined in Eqs. (8) and (10). For a simple fully covariant theory, a bare $\gamma^{\mu}$ coupling fulfills current conservation [19,21]. However, as it is shown in Appendix C, within our approximations a bare coupling produces divergent matrix elements of the current, and therefore we do not consider this term in the present paper.

\section{APPROXIMATING THE TRIANGLE DIAGRAM ON THE LIGHT FRONT}

Our aim is to retain the essential physics contained in the triangle diagram (Fig. 1) and to be able to use the Hamiltonian wave-function language in order to retain the successfully nonperturbative phenomenology developed within this framework. At the same time we wish to go beyond a simple valence description. To accomplish these goals and to eliminate the relative light-front time between the quarks, we perform the $k^{-}$integration in Eq. (3) with some assumptions on the analytical structure of the $\Lambda$ and $\Gamma$ vertices for the pion and the photon. To be more precise, Eq. (3) is evaluated with the assumptions that: (i) the momentum components, $\Lambda(k, P)$, of the vertex functions, both for the pion and the vector mesons, vanish in the complex-plane $k^{-}$for $\left|k^{-}\right| \rightarrow \infty$; and (ii) the contributions of the singularities of $\Lambda(k, P)$ can be neglected. Furthermore, the Dirac structures of the vector-meson vertex function, $\hat{V}_{n}^{\mu}(k, k-q)$, are assumed to be regular functions of the complex variable $k^{-}$. The expressions for $\hat{V}_{n}^{\mu}\left(k, k^{\prime}\right)$ given by Eqs. (14) or (16) obviously fulfill the requirement that no pole is present in the $k^{-}$complex plane.

Obviously the assumption (ii) implies that some interesting physics may be missing. In order to have an idea of the relevance of assumption (ii), we tested this assumption by an evaluation of the spacelike pion form factor within the analytic model of Ref. [21] for the Bethe-Salpeter amplitude. We checked that in a reference frame where $q^{+}=0$ the contribution of the poles of $\Lambda(k, P)$ is a large one, while in a reference frame where $q^{+}>0$ the pion form factor obtained neglecting the poles of $\Lambda(k, P)$ is quite reasonable, especially for $Q^{2}=-q^{2} \geq 2(\mathrm{GeV} / c)^{2}$. Therefore, the application of assumption (ii) appears very effective in a reference frame where $q^{+}>0$. We leave a deeper exploration of this point to another work.

Explicit integration over $k^{-}$with the above mentioned assumptions is performed in Appendix D. It is worth noting that the expected kinematical constraints on $k^{+}$, which allow the separation of valence and nonvalence contributions, are straightforwardly obtained through the $k^{-}$integration. For the sake of clarity, the timelike case and the spacelike case are treated separately.

\section{A. Timelike case}

In the timelike case, one has $q^{\mu}=P_{\pi}^{\mu}+P_{\bar{\pi}}^{\mu}$, and $q^{+}>$ 0 . Equation (3) written in light-front variables and integrated over $k^{-}$becomes

$$
\begin{aligned}
j^{\mu}= & \frac{e}{(2 \pi)^{3}} \frac{m^{2}}{f_{\pi}^{2}} N_{c} \int_{0}^{q^{+}} \frac{d k^{+} d \mathbf{k}_{\perp}}{\left(k^{+}-P_{\pi}^{+}\right) k^{+}\left(q^{+}-k^{+}\right)} \\
& \times\left\{\Theta\left(P_{\pi}^{+}-k^{+}\right) I_{1}^{\mu}+\Theta\left(k^{+}-P_{\pi}^{+}\right) I_{2}^{\mu}\right\},
\end{aligned}
$$

where the quantities $I_{1}^{\mu}$ and $I_{2}^{\mu}$ in Eq. (17) are defined as follows:

$$
\begin{aligned}
I_{1}^{\mu}= & {\left[\bar{\Lambda}_{\pi}\left(k, P_{\pi}\right) \Lambda_{\bar{\pi}}\left(k-P_{\pi}, P_{\bar{\pi}}\right)\right]_{k^{-}=k_{\mathrm{on}}^{-}}\left[T_{\text {on },(1)}^{\mu}\right.} \\
& \left.+T_{1,(1)}^{\mu}+T_{2,(1)}^{\mu}\right], \\
I_{2}^{\mu}=[ & \left.\bar{\Lambda}_{\pi}\left(k, P_{\pi}\right) \Lambda_{\bar{\pi}}\left(k-P_{\pi}, P_{\bar{\pi}}\right)\right]_{k^{-}=q^{-}+(k-q)_{\mathrm{on}}^{-}}\left[T_{\text {on },(2)}^{\mu}\right. \\
& \left.+T_{1,(2)}^{\mu}+T_{3,(2)}^{\mu}\right] .
\end{aligned}
$$

The explicit expressions for the quantities $T_{\text {on,(1) }}^{\mu}, T_{\text {on,(2), }}^{\mu}$, and for the instantaneous terms $T_{1,(i)}^{\mu}(i=1,2), T_{2,1}^{\mu}, T_{3,2}^{\mu}$ can be found in Appendix D [see Eqs. (D18)-(D22), respectively].

The first term of Eq. (17), with $k^{+}-P_{\pi}^{+} \leq 0$, and the second term, with $k^{+}-P_{\pi}^{+} \geq 0$, are represented in Fig. 2 by the diagrams (a), with the arrow of $k^{+}-P_{\pi}^{+}$from the left to the right, and (b) with the arrow of $k^{+}-P_{\pi}^{+}$from the right to the left, respectively. In the first term only the vertex function $\bar{\Lambda}_{\pi}\left(k, P_{\pi}\right)$ has the momentum fraction $k^{+} / P_{\pi}^{+}$in the valence-sector range $[0,1]$ and in the second 
term only the vertex function $\Lambda_{\bar{\pi}}\left(k-P_{\pi}, P_{\bar{\pi}}\right)$ has the momentum fraction $\left(k^{+}-P_{\pi}^{+}\right) / P_{\bar{\pi}}^{+}$in the valence-sector range $[0,1]$. The vertex functions in the valence range give the possibility to establish the desired bridge between Bethe-Salpeter and Hamiltonian languages, as explained in detail in Sec. IV.

\section{B. Spacelike case}

The expression for the triangle diagram in the spacelike case, where $P_{\pi^{\prime}}^{\mu}=P_{\pi}^{\mu}+q^{\mu}$, can be obtained from Eq. (3) replacing $-P_{\pi}^{\pi^{\prime}}$ with $P_{\pi}^{\mu}, \bar{\pi}$ with $\pi^{\prime}$ and the pion vertices $\bar{\Lambda}_{\pi}\left(k, P_{\pi}\right)$ and $\Lambda_{\bar{\pi}}\left(k-P_{\pi}, P_{\bar{\pi}}\right)$ with $\Lambda_{\pi}\left(-k, P_{\pi}\right)$ and $\bar{\Lambda}_{\pi^{\prime}}\left(k+P_{\pi}, P_{\pi^{\prime}}\right)$, respectively. Then, after the $k^{-}$integration with the assumption $q^{+} \geq 0$, the triangle diagram can be decomposed as follows (see Appendix D):

$$
j^{\mu}=j^{(\mathrm{I}) \mu}+j^{(\mathrm{II}) \mu},
$$

where $j^{(\mathrm{I}) \mu}$ has the integration on $k^{+}$constrained by $-P_{\pi}^{+} \leq k^{+} \leq 0$ [diagram (a) of Fig. 3], while $j^{(\mathrm{II}) \mu}$ has the integration on $k^{+}$in the interval $0<k^{+}<q^{+}$ [diagram (b) of Fig. 3]. The valence component of the pion contributes to $j^{(\mathrm{I}) \mu}$ only, while $j^{(\mathrm{II}) \mu}$ is the contribution of the pair-production mechanism from an incoming virtual photon with $q^{+}>0[14,19,21,22,26,27,41]$.

\section{Valence region contribution}

Let us define $k^{\prime}=k+P_{\pi}$, with $\left(k^{\prime+}, \mathbf{k}_{\perp}^{\prime}\right)$ the light-front momentum of a quark in the valence range. Then $j^{(\mathrm{I}) \mu}$ can be written in the following form

$$
\begin{aligned}
j^{(\mathrm{I}) \mu}= & \frac{e}{(2 \pi)^{3}} \frac{m^{2}}{f_{\pi}^{2}} N_{c} \int_{0}^{P_{\pi}^{+}} \frac{d k^{\prime+} d \mathbf{k}_{\perp}^{\prime}}{\left(k^{\prime+}-P_{\pi}^{+}\right) k^{\prime+}\left(P_{\pi^{\prime}}^{+}-k^{\prime+}\right)} \\
& \times\left[T_{\mathrm{on},(4)}^{\prime \mu}+T_{2,(4)}^{\prime \mu}+T_{3,(4)}^{\prime \mu}\right] \bar{\Lambda}_{\pi \prime}\left(k^{\prime}, P_{\pi \prime}\right) \\
& \left.\times \Lambda_{\pi}\left(P_{\pi}-k^{\prime}, P_{\pi}\right)\right]_{k^{\prime-}=k_{\mathrm{on}}^{\prime-}},
\end{aligned}
$$

where

$$
k_{\mathrm{on}}^{\prime-}=\frac{\left(\mathbf{k}_{\perp}^{\prime 2}+m^{2}\right)}{k^{\prime+}} .
$$

The explicit expressions for the quantity $T_{\mathrm{on},(4)}^{\prime \mu}$, and for the instantaneous terms $T_{2,(4)}^{\prime \mu}$ and $T_{3,(4)}^{\prime \mu}$ can be found in Appendix D [Eqs. (D47)-(D49), respectively].

In Eq. (21) both the vertex functions have the quark momentum fractions $k^{\prime+} / P_{\pi^{\prime}}^{+}$and $\left(P_{\pi}-k^{\prime}\right)^{+} / P_{\pi}^{+}$in the valence-sector range $[0,1]$. Note that the on-shell momenta in Eq. (D47) allow one to retrieve the relativistic spin coupling factors with the spin $1 / 2$ Melosh rotations automatically included $[8,9,50]$ (see the following section).

\section{Pair-production contribution}

As shown in Appendix D, the pair-production contribution can be written in the following form

$$
\begin{aligned}
j^{(\mathrm{II}) \mu}= & -\frac{e}{(2 \pi)^{3}} \frac{m^{2}}{f_{\pi}^{2}} N_{c} \int_{0}^{q^{+}} \frac{d k^{+} d \mathbf{k}_{\perp}}{\left(k^{+}+P_{\pi}^{+}\right) k^{+}\left(q^{+}-k^{+}\right)} \\
& \times\left[T_{\mathrm{on},(2)}^{\prime \mu}+T_{1,(2)}^{\prime \mu}+T_{3,(2)}^{\prime \mu}\right]\left[\bar{\Lambda}_{\pi^{\prime}}\left(k+P_{\pi}, P_{\pi^{\prime}}\right)\right. \\
& \left.\times \Lambda_{\pi}\left(-k, P_{\pi}\right)\right]_{k^{-}=q^{-}+(k-q)_{\mathrm{on}}^{-}} .
\end{aligned}
$$

The quantity $T_{\text {on,(2) }}^{\prime \mu}$ and the instantaneous terms $T_{1,(2)}^{\prime \mu}$ and $T_{3,(2)}^{\prime \mu}$ are defined in Appendix D by Eqs. (D50)-(D52), respectively.

In Eq. (23) only the vertex function $\bar{\Lambda}_{\pi^{\prime}}$ has the quark momentum fraction $\left(P_{\pi}+k\right)^{+} / P_{\pi^{\prime}}^{+}$in the range $[0,1]$.

\section{VALENCE COMPONENT OF THE LIGHT-FRONT WAVE FUNCTION}

In order to establish a bridge between the Bethe-Salpeter language and the Hamiltonian wave-function language and thus to exploit the nonperturbative phenomenology developed within the Hamiltonian approach, in this section we try to obtain a link between the Bethe-Salpeter amplitudes in the valence range and the HLFD wave functions.

\section{A. Meson wave functions}

\section{Pion}

To fully interpret the terms that appear in Eqs. (17), (21), and (23), we have to discuss valence and nonvalence components of the light-front wave function. Let us first begin with the valence component, which can be defined starting from the Bethe-Salpeter amplitude [51]. In the present model the pion Bethe-Salpeter amplitude is given by

$$
\begin{aligned}
\Psi_{\pi}\left(k, P_{\pi}\right)= & \frac{m}{f_{\pi}} \frac{\not k+m}{k^{2}-m^{2}+\imath \epsilon} \gamma^{5} \Lambda_{\pi}\left(k, P_{\pi}\right) \\
& \times \frac{\not k-\not P_{\pi}+m}{\left(k-P_{\pi}\right)^{2}-m^{2}+\imath \epsilon},
\end{aligned}
$$

where the pion vertex is $\frac{m}{f_{\pi}} \gamma^{5} \Lambda_{\pi}\left(k, P_{\pi}\right)$.

The valence component of the light-front wave function can be obtained from the Bethe-Salpeter amplitude (24) in the valence-sector range, $0 \leq k^{+} \leq P_{\pi}^{+}$, disregarding the instantaneous terms in Eq. (24), multiplying $\Psi_{\pi}$ by the factor $\left[k^{+}\left(k^{+}-P_{\pi}^{+}\right)\right] /(2 \pi l)$ and integrating over $k^{-}$:

$$
\begin{aligned}
\phi_{\pi}\left(k^{+}, \vec{k}_{\perp} ; P_{\pi}^{+}, \vec{P}_{\pi \perp}\right)= & -\imath \frac{m}{f_{\pi}} k^{+}\left(k^{+}-P_{\pi}^{+}\right) \int \frac{d k^{-}}{2 \pi} \\
& \times \frac{k_{\mathrm{on}}+m}{k^{2}-m^{2}+\imath \epsilon} \gamma^{5} \Lambda_{\pi}\left(k, P_{\pi}\right) \\
& \times \frac{\left(k-\not P_{\pi}\right)_{\mathrm{on}}+m}{\left(k-P_{\pi}\right)^{2}-m^{2}+\imath \epsilon} .
\end{aligned}
$$

Two poles, $k_{(1)}$ and $k_{(3)}$, appear in Eq. (25), respectively, in the lower and in the upper $k^{-}$semiplanes. We perform the $k^{-}$integration in the lower complex semiplane disregarding the contributions that arise from the singularities 
of the vertex $\Lambda_{\pi}\left(k, P_{\pi}\right)$ [cf. assumptions (i) and (ii) at the beginning of Sec. III]. Then the pion wave function becomes

$$
\phi_{\pi}\left(k^{+}, \mathbf{k}_{\perp} ; P_{\pi}^{+}, \mathbf{P}_{\pi \perp}\right)=\frac{m}{f_{\pi}}\left(k_{\text {on }}+m\right) \gamma^{5} \frac{P_{\pi}^{+}\left[\Lambda_{\pi}\left(k, P_{\pi}\right)\right]_{\left[k^{-}=k_{\mathrm{on}}^{-}\right]}}{\left[m_{\pi}^{2}-M_{0}^{2}\left(k^{+}, \mathbf{k}_{\perp} ; P_{\pi}^{+}, \mathbf{P}_{\pi \perp}\right)\right]}\left[\left(k-\not P_{\pi}\right)_{\text {on }}+m\right] .
$$

If the $k^{-}$integration is done in the upper semiplane within the same assumptions, one has

$$
\phi_{\pi}\left(k^{+}, \mathbf{k}_{\perp} ; P_{\pi}^{+}, \mathbf{P}_{\pi \perp}\right)=\frac{m}{f_{\pi}}\left(k_{\mathrm{on}}+m\right) \gamma^{5} \frac{P_{\pi}^{+}\left[\Lambda_{\pi}\left(k, P_{\pi}\right)\right]_{\left[k^{-}=P_{\pi}^{-}-\left(P_{\pi}-k\right)_{\mathrm{o}}^{-}\right]}}{\left[m_{\pi}^{2}-M_{0}^{2}\left(P_{\pi}^{+}-k^{+}, \mathbf{P}_{\pi \perp}-\mathbf{k}_{\perp} ; P_{\pi}^{+}, \mathbf{P}_{\pi \perp}\right)\right]}\left[\left(k-\not P_{\pi}\right)_{\mathrm{on}}+m\right] .
$$

In principle, the elimination of the relative light-front time between the quark and the antiquark in the pion Bethe-Salpeter amplitude by the $k^{-}$integration in Eq. (25), should give a unique answer, which defines the valence component of the wave function in the range $0 \leq k^{+} \leq P_{\pi}^{+}$, with both the quarks on their mass shell. Therefore in order to require consistency within our model, we will assume $\left[\Lambda_{\pi}\left(k, P_{\pi}\right)\right]_{\left[k^{-}=k_{\text {on }}^{-}\right]}$and $\left[\Lambda_{\pi}\left(k, P_{\pi}\right)\right]_{\left[k^{-}=P_{\pi}^{-}-\left(P_{\pi}-k\right)_{\text {on }}^{-}\right]}$to be equal in that kinematical range [note that $M_{0}^{2}\left(k^{+}, \mathbf{k}_{\perp} ; P_{\pi}^{+}, \mathbf{P}_{\pi \perp}\right)$ is equal to $\left.M_{0}^{2}\left(P_{\pi}^{+}-k^{+}, \mathbf{P}_{\pi \perp}-\mathbf{k}_{\perp} ; P_{\pi}^{+}, \mathbf{P}_{\pi \perp}\right)\right]$. This assumption produces a momentum component of the valence lightfront wave-function symmetrical for the exchange of the quark momenta, since the vertex function $\Lambda_{\pi}\left(k, P_{\pi}\right)$ is assumed to be symmetrical.

Within a Bethe-Salpeter approach, the function $\phi_{\pi}$ fulfills a two-body Schrödinger-like equation, with the proper Melosh structure represented by the matrix $\left(k_{\text {on }}+\right.$ $m) \gamma^{5}\left[\left(k-\not{ }_{\pi}\right)_{\text {on }}+m\right][8]$. Therefore, when the plus component of the quark momentum is in the interval $0 \leq k^{+} \leq$ $P_{\pi}^{+}$, the function $\phi_{\pi}$ will be substituted in our approach with the HLFD pion wave function, with momentum component $\psi_{\pi}\left(k^{+}, \mathbf{k}_{\perp} ; P_{\pi}^{+}, \mathbf{P}_{\pi \perp}\right)$ (see Ref. [8]):

$$
\begin{aligned}
\phi_{\pi}\left(k^{+}, \mathbf{k}_{\perp} ; P_{\pi}^{+}, \mathbf{P}_{\pi \perp}\right)= & \left(\not_{\mathrm{on}}+m\right) \gamma^{5}\left[\left(\not k-\not P_{\pi}\right)_{\mathrm{on}}+m\right] \\
& \times \psi_{\pi}\left(k^{+}, \mathbf{k}_{\perp} ; P_{\pi}^{+}, \mathbf{P}_{\pi \perp}\right) .
\end{aligned}
$$

This substitution represents the required bridge announced in Sec. I. Through this relation, we can embed in our approach the nonperturbative features implicitly contained in the model meson wave functions. Indeed, we use eigenstates of a Hamiltonian which describes the essential features of the experimental spectra.

\section{Vector meson}

In analogy with the pion case, one can define the lightfront VM wave function, which describes the valence component of the meson state $|n \lambda\rangle$. Indeed, starting from the Bethe-Salpeter amplitude for a vector meson

$$
\begin{aligned}
\Psi_{n \lambda}\left(k, P_{n}\right)= & \frac{\not k+m}{k^{2}-m^{2}+\imath \epsilon}\left[\epsilon_{\lambda}\left(P_{n}\right) \cdot \hat{V}_{n}\left(k, k-P_{n}\right)\right] \\
& \times \Lambda_{n}\left(k, P_{n}\right) \frac{\not k-\not P_{n}+m}{\left(k-P_{n}\right)^{2}-m^{2}+\imath \epsilon},
\end{aligned}
$$

the valence component of the light-front wave function can be defined from $\Psi_{n \lambda}\left(k, P_{n}\right)$ integrating over $k^{-}$, disregarding the instantaneous terms and multiplying by the factor $\left[k^{+}\left(k^{+}-P_{\pi}^{+}\right)\right] /(2 \pi \imath)$, as we already did for the pion. Furthermore, in this case one has to take on their mass shell both the quark momenta in the Dirac structure of the VM vertex function, $\hat{V}_{n}\left(k, k-P_{n}\right)$ :

$$
\begin{aligned}
\phi_{n \lambda}\left(k^{+}, \vec{k}_{\perp} ; P_{n}^{+}, \vec{P}_{n \perp}\right)= & -\imath k^{+}\left(k^{+}-P_{n}^{+}\right) \int \frac{d k^{-}}{2 \pi} \frac{k_{\mathrm{on}}+m}{k^{2}-m^{2}+\imath \epsilon} \Lambda_{n}\left(k, P_{n}\right)\left[\epsilon_{\lambda}\left(P_{n}\right) \cdot\left[\hat{V}_{n}\left(k, k-P_{n}\right)\right]_{\mathrm{on}}\right] \\
& \times \frac{\left(k-\not P_{n}\right)_{\mathrm{on}}+m}{\left(k-P_{n}\right)^{2}-m^{2}+\imath \epsilon}
\end{aligned}
$$

where $\left[\hat{V}_{n}\left(k, k-P_{n}\right)\right]_{\text {on }}$ is defined by Eq. (16) in order to retrieve the ${ }^{3} S_{1}$ vector-meson vertex of Ref. [8]. Assuming that $\Lambda_{n}\left(k, P_{n}\right)$ does not diverge in the complex plane $k^{-}$for $\left|k^{-}\right| \rightarrow \infty$, and neglecting the contributions of its singularities in the $k^{-}$integration, the valence VM wave function is

$$
\phi_{n \lambda}\left(k^{+}, \mathbf{k}_{\perp} ; P_{n}^{+}, \mathbf{P}_{n \perp}\right)=P_{n}^{+}\left(k_{\mathrm{on}}+m\right) \frac{\left[\boldsymbol{\epsilon}_{\lambda}\left(P_{n}\right) \cdot\left[\hat{V}_{n}\left(k, k-P_{n}\right)\right]_{\mathrm{on}}\right]}{\left[M_{n}^{2}-M_{0}^{2}\left(k^{+}, \mathbf{k}_{\perp} ; P_{n}^{+}, \mathbf{P}_{n \perp}\right)\right]}\left[\Lambda_{n}\left(k, P_{n}\right)\right]_{\left[k^{-}=k_{\mathrm{on}}^{-}\right.}\left[\left(k-\not k{ }_{n}\right)_{\mathrm{on}}+m\right] .
$$

In analogy with the pion case, we assume $\left[\Lambda_{n}\left(k, P_{n}\right)\right]_{\left[k^{-}=k_{\text {on }}^{-}\right]}=\left[\Lambda_{n}\left(k, P_{n}\right)\right]_{\left[k^{-}=P_{n}^{-}-\left(P_{n}-k\right)_{\text {on }}^{-}\right]}$in the valence-sector range, $0 \leq k^{+} \leq P_{n}^{+}$.

As for the pion, the function $\phi_{n \lambda}$, with the plus component of the quark momentum in the interval $0 \leq k^{+} \leq P_{\pi}^{+}$, will be substituted with the HLFD vector-meson wave function, with momentum component $\psi_{n}\left(k^{+}, \mathbf{k}_{\perp} ; P_{n}^{+}, \mathbf{P}_{n \perp}\right)$ (with $M_{n} \rightarrow$ $\left.M_{0}\right)$, [8]:

$$
\phi_{n \lambda}\left(k^{+}, \mathbf{k}_{\perp} ; P_{n}^{+}, \mathbf{P}_{n \perp}\right)=\left(k_{\mathrm{on}}+m\right)\left[\epsilon_{\lambda}\left(P_{n}\right) \cdot\left[\hat{V}_{n}\left(k, k-P_{n}\right)\right]_{\mathrm{on}}\right]\left[\left(k-\not P_{n}\right)_{\mathrm{on}}+m\right] \psi_{n}\left(k^{+}, \mathbf{k}_{\perp} ; P_{n}^{+}, \mathbf{P}_{n \perp}\right) .
$$


In conclusion, Eqs. (26),(28) and (31), (32) establish a link between the momentum part of the meson HLFD wave functions and the momentum part of the meson vertex functions.

The valence component of the VM wave function are normalized to the probability of the valence component of the meson state $|n \lambda\rangle$ (see Appendix E). This probability is estimated in a schematic model in Appendix F.

The corresponding normalization for the pion wave function is included in an overall normalization constant for the pion form factor.

\section{B. Photon wave function}

One can define as well the valence component of the hadronic contribution to the photon wave function, starting from the Bethe-Salpeter amplitude of the photon, which can be written as

$$
\Psi_{\gamma}^{\mu}(k, q)=\frac{k+m}{k^{2}-m^{2}+\imath \epsilon} \Gamma^{\mu}(k, q) \frac{k-\not k+m}{(k-q)^{2}-m^{2}+\imath \epsilon},
$$

where $\Gamma^{\mu}(k, q)$ is the photon-vertex amplitude [see Eq. (6)].

In analogy with Eq. (30), the valence component of the virtual-photon light-front wave function can be obtained from the Bethe-Salpeter amplitude (33) in the valence sector, $0 \leq k^{+} \leq q^{+}$, separating out the instantaneous terms of Eq. (33), integrating in $k^{-}$and multiplying by the factor $\left[k^{+}\left(k^{+}-P_{\pi}^{+}\right)\right] /(2 \pi \imath)$.

Then, using our explicit expression for $\Gamma^{\mu}(k, q)$ given by Eq. (8), the light-front wave function of the photon can be defined by

$$
\begin{aligned}
\phi_{\gamma}^{\mu}\left(k^{+}, \mathbf{k}_{\perp} ; q^{2}, q^{+}, \mathbf{q}_{\perp}\right)= & -\imath k^{+}\left(k^{+}-q^{+}\right) \int \frac{d k^{-}}{2 \pi} \\
& \times \frac{k_{\mathrm{on}}+m}{k^{2}-m^{2}+\imath \epsilon}\left[\Gamma^{\mu}(k, q)\right]_{\mathrm{on}} \\
& \times \frac{(k-\not)_{\mathrm{on}}+m}{(k-q)^{2}-m^{2}+\imath \epsilon},
\end{aligned}
$$

where the label "on" in $\left[\Gamma^{\mu}(k, q)\right]_{\text {on }}$ means that, as in the VM case, the Dirac structures of the photon-vertex amplitude, $\Gamma^{\mu}(k, q)$, have to be taken with both the quark momenta on their mass shell. Therefore, in analogy with Eq. (30), a possible choice for the quantity $\hat{V}_{n}(k, k-q)$ of Eq. (8) is given by the quantity $\left[\hat{V}_{n}(k, k-q)\right]_{\text {on }}$ as defined by Eq. (16).

Then, performing the $k^{-}$integration with the assumptions given at the beginning of Sec. III, in the range $0 \leq$ $k^{+} \leq q^{+}$Eq. (34) becomes

$$
\phi_{\gamma}^{\mu}\left(k^{+}, \mathbf{k}_{\perp} ; q^{2}, q^{+}, \mathbf{q}_{\perp}\right)=\left(k_{\mathrm{on}}+m\right) \psi_{\gamma}^{\mu}\left(k^{+}, \mathbf{k}_{\perp} ; q^{2}, q^{+}, \mathbf{q}_{\perp}\right)\left[(k-\not)_{\mathrm{on}}+m\right],
$$

where the function $\psi_{\gamma}^{\mu}\left(k^{+}, \mathbf{k}_{\perp} ; q^{2}, q^{+}, \mathbf{q}_{\perp}\right)$, which includes the Dirac structures of the photon vertex, is defined by

$$
\psi_{\gamma}^{\mu}\left(k^{+}, \mathbf{k}_{\perp} ; q^{2}, q^{+}, \mathbf{q}_{\perp}\right)=\left[\Gamma^{\mu}(k, q)\right]_{\mathrm{on}} \frac{q^{+}}{\left[q^{2}-M_{0}^{2}\left(k^{+}, \mathbf{k}_{\perp} ; q^{+}, \mathbf{q}_{\perp}\right)+\imath \epsilon\right]} .
$$

As in the previous meson case, to have consistency in our virtual-photon wave-function model, we will not distinguish between $\left[\Lambda_{n}(k, q)\right]_{\left[k^{-}=k_{\text {on }}^{-}\right]}$and $\left[\Lambda_{n}(k, q)\right]_{\left[k^{-}=q^{-}-(q-k)_{\text {on }}^{-}\right]}$in the valence sector, $0 \leq k^{+} \leq$ $q^{+}$. Therefore we obtain for $\psi_{\gamma}^{\mu}\left(k^{+}, \mathbf{k}_{\perp} ; q^{2}, q^{+}, \mathbf{q}_{\perp}\right)$ the same result when the $k^{-}$integration is performed both in the lower or in the upper $k^{-}$complex semiplane.

The valence wave function, $\phi_{\gamma}^{\mu}\left(k^{+}, \mathbf{k}_{\perp} ; q^{2}, q^{+}, \mathbf{q}_{\perp}\right)$, and the function $\psi_{\gamma}^{\mu}\left(k^{+}, \mathbf{k}_{\perp} ; q^{2}, q^{+}, \mathbf{q}_{\perp}\right)$ depend on the value of $q^{2}$ carried by the virtual photon. Note that in the timelike case a singularity appears in the photon valence wave function [see Eq. (36)].
If, as in Eq. (10), the photon vertex $\left[\Gamma^{\mu}(k, q)\right]_{\text {on }}$ is taken with on-shell quantities for the vector mesons in the numerator, i.e. if we take

$$
\begin{aligned}
{\left[\Gamma^{\mu}(k, q)\right]_{\text {on }}=} & \sqrt{2} \sum_{n, \lambda} \epsilon_{\lambda}\left(P_{n}\right) \cdot\left[\hat{V}_{n}\left(k, k-P_{n}\right)\right]_{\text {on }} \\
& \times\left[\Lambda_{n}\left(k, P_{n}\right)\right]_{\left[k^{-}=k_{\text {on }}^{-}\right]} \\
& \times \frac{\left[\epsilon_{\lambda}^{\mu}\left(P_{n}\right)\right]^{*} f_{V n}}{\left[q^{2}-M_{n}^{2}+\imath M_{n} \tilde{\Gamma}_{n}\left(q^{2}\right)\right]}
\end{aligned}
$$

and identify Eqs. (31) and (32), then the function $\psi_{\gamma}^{\mu}\left(k^{+}, \mathbf{k}_{\perp} ; q^{2}, q^{+}, \mathbf{q}_{\perp}\right)$ can be written as follows:

$$
\begin{aligned}
\psi_{\gamma}^{\mu}\left(k^{+}, \mathbf{k}_{\perp} ; q^{2}, q^{+}, \mathbf{q}_{\perp}\right)= & \sqrt{2} \sum_{n, \lambda}\left[\epsilon_{\lambda}\left(P_{n}\right) \cdot\left[\hat{V}_{n}\left(k, k-P_{n}\right)\right]_{\mathrm{on}}\right] \frac{\left[M_{n}^{2}-M_{0}^{2}\left(k^{+}, \mathbf{k}_{\perp} ; P_{n}^{+}, \mathbf{P}_{n \perp}\right)\right]}{\left[q^{2}-M_{0}^{2}\left(k^{+}, \mathbf{k}_{\perp} ; q^{+}, \mathbf{q}_{\perp}\right)+\imath \epsilon\right]} \psi_{n}\left(k^{+}, \mathbf{k}_{\perp} ; P_{n}^{+}, \mathbf{P}_{n \perp}\right) \\
& \times \frac{\left[\epsilon_{\lambda}^{\mu}\left(P_{n}\right)\right]^{*} f_{V n}}{\left[q^{2}-M_{n}^{2}+\imath M_{n} \tilde{\Gamma}_{n}\left(q^{2}\right)\right]}
\end{aligned}
$$


in terms of the momentum part of the HLFD vector-meson wave functions, $\psi_{n}\left(k^{+}, \mathbf{k}_{\perp} ; P_{n}^{+}, \mathbf{P}_{n \perp}\right)$.

\section{CONTRIBUTION OF NONVALENCE COMPONENTS TO THE CURRENT}

In this section we wish settle our treatment for the nonvalence components through a simple approximation for the vertex functions outside the valence range.

\section{A. Timelike case: The photon nonvalence component}

The process of pion-antipion production is shown in Fig. 2, where the dashed lines [both in (a) and in (b)] represent two different light-front times. At the first time (the one on the right) the hadronic valence component of the virtual photon is represented, while at the second one the $2 q 2 \bar{q}$ photon nonvalence component is depicted (see also Fig. 5). The two parts of Fig. 2, i.e. (a) and (b), differ by the emission vertex of an antipion or of a pion [see also Fig. 5(b)], respectively. The corresponding quark amplitudes for the radiation of an antipion or a pion are given in Eq. (17) by the antipion vertex $\Lambda_{\bar{\pi}}\left(k-P_{\pi} ; P_{\bar{\pi}}\right)$, evaluated at $k^{-}=k_{\text {on }}^{-}$for $\left(k-P_{\pi}\right)^{+}<0$, and by the pion vertex $\bar{\Lambda}_{\pi}\left(k ; P_{\pi}\right)$, evaluated at $k^{-}=q^{-}+(k-q)_{\text {on }}^{-}$for $k^{+}>$ $P_{\pi}^{+}$, respectively, i.e. outside the valence range.

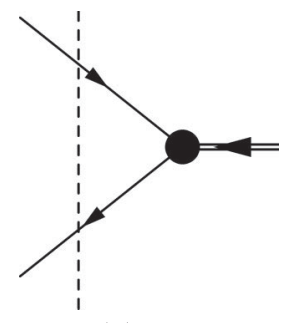

(a)

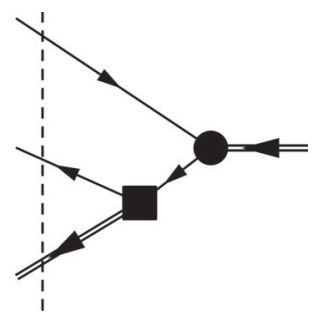

(b)
FIG. 5. (a) Valence component, $|q \bar{q}\rangle$, of the wave function of an incoming system (photon or meson). (b) Nonvalence component, $|q \bar{q} q \bar{q}\rangle$, of the wave function of the same incoming system. The extra $q \bar{q}$ pair is radiatively emitted by a quark in the valence component.

Once the interaction that couples the valence to the $2 q 2 \bar{q}$ component is known (see Fig. 2), the amplitude for the photon decay in a $\pi \bar{\pi}$ pair can be constructed. To this end, let us introduce a kernel operator $\mathcal{K}$ which realizes this coupling. Then, we can write the following equation to relate the valence component of the pion wave function, $\psi_{\pi}\left(k^{\prime+}, \mathbf{k}_{\perp}^{\prime} ; P_{\pi}^{+}, \mathbf{P}_{\pi \perp}\right)$, to the vertex function $\bar{\Lambda}_{\pi}\left(k ; P_{\pi}\right)$ at $k^{-}=q^{-}+(k-q)_{\text {on }}^{-}$, which is the amplitude for the pion emission [see Figs. 2(b) and 6]:

$$
\begin{aligned}
\overline{\mathcal{D}}_{\pi} & :=\frac{m}{f_{\pi}} \bar{\Lambda}_{\pi}\left(k ; P_{\pi}\right)_{\left[k^{-}=q^{-}+(k-q)_{\text {on }}^{-}\right]} \\
& =\frac{1}{4} \sum_{\alpha^{\prime} \beta^{\prime} \alpha \beta} \int_{0}^{P_{\pi}^{+}} \frac{d k^{\prime+} d \mathbf{k}_{\perp}^{\prime}}{k^{\prime+}\left(P_{\pi}^{+}-k^{\prime+}\right)} \psi_{\pi}^{*}\left(k^{\prime+}, \mathbf{k}_{\perp}^{\prime} ; P_{\pi}^{+}, \mathbf{P}_{\pi \perp}\right)\left(\gamma^{5}\right)^{\beta \alpha}\left(\gamma^{5}\right)_{\beta^{\prime} \alpha^{\prime}} \mathcal{K}_{\alpha \beta}^{\alpha^{\prime} \beta^{\prime}}\left(k^{\prime+}, \mathbf{k}_{\perp}^{\prime} ; k^{+}, \mathbf{k}_{\perp} ; q^{-}, q^{+}, \mathbf{q}_{\perp}\right) .
\end{aligned}
$$

For simplicity, the example of a $\gamma^{5}$ structure was used in Eq. (39), just to be consistent with our assumption of a pseudoscalar pion model.

One can write an analogous expression for the emission of $\bar{\pi}$ :

$$
\begin{aligned}
\mathcal{D}_{\bar{\pi}} & :=\frac{m}{f_{\pi}}\left[\Lambda_{\bar{\pi}}\left(k-P_{\pi} ; P_{\bar{\pi}}\right)\right]_{\left(k^{-}=k_{\mathrm{on}}^{-}\right)} \\
& =\frac{1}{4} \sum_{\alpha^{\prime} \beta^{\prime} \alpha \beta} \int_{0}^{P_{\bar{\pi}}^{+}} \frac{d k^{\prime+} d \mathbf{k}_{\perp}^{\prime}}{k^{\prime+}\left(P_{\pi}^{+}-k^{\prime+}\right)} \psi_{\bar{\pi}}^{*}\left(k^{\prime+}, \mathbf{k}_{\perp}^{\prime} ; P_{\bar{\pi}}^{+}, \mathbf{P}_{\bar{\pi} \perp}\right)\left(\gamma^{5}\right)^{\beta \alpha}\left(\gamma^{5}\right)_{\beta^{\prime} \alpha^{\prime}} \mathcal{K}_{\alpha \beta}^{\alpha^{\prime} \beta^{\prime}}\left(k^{\prime+}, \mathbf{k}_{\perp}^{\prime} ; k^{+}-P_{\pi}^{+}, \mathbf{k}_{\perp}-\mathbf{P}_{\pi \perp} ; q^{-}, q^{+}, \mathbf{q}_{\perp}\right) .
\end{aligned}
$$

In our model calculation, both pion emission vertices will be substituted by a constant, following Ref. [18].

\section{B. Spacelike case: the pion nonvalence component}

In the spacelike region, for $q^{+}>0$ the nonvalence component of the final pion wave function appears in both the two contributions of the current obtained after the $k^{-}$ integration, and given by Eqs. (21) and (23) [see diagrams (b) and (c) in Fig. 7]. On one hand the valence component of the final pion is coupled to the nonvalence $2 q 2 \bar{q}$ component [see Fig. 7(b)], through an interaction kernel $\mathcal{H}$, which contributes to the quark-photon absorption vertex of Eq. (D36), given by $\Gamma^{\mu}(4)=\Gamma^{\mu}\left(k^{\prime}-P_{\pi}, q\right)$

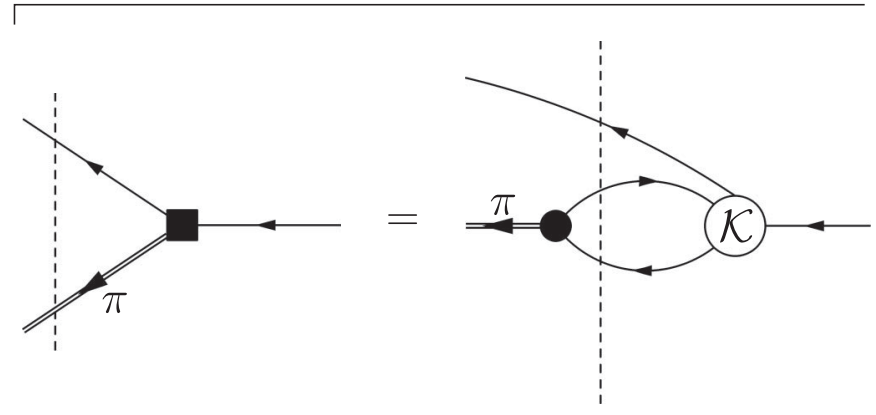

FIG. 6. Virtual decay amplitude for a pion emission from a quark ( $q \rightarrow q \pi)$ produced by the operator $\mathcal{K}$, see Eq. (39). The analogous diagram for pion absorption by a quark can be easily obtained by replacing the final pion leg with an initial pion leg. 
with $k^{\prime-}=k_{\text {on }}^{\prime-}$. On the other hand the vertex $\Lambda_{\pi}\left(-k ; P_{\pi}\right)$ in Eq. (23), evaluated at $k^{-}=q^{-}+(k-q)_{\text {on }}^{-}$for $-k^{+}<$ 0 , describes the quark-pion absorption through another interaction kernel $\mathcal{K}^{\prime}$ and generates the nonvalence $2 q 2 \bar{q}$ component of the final pion [see Fig. 7(c)]. We identify the kernel $\mathcal{K}^{\prime}$ with the kernel $\mathcal{K}$, already used in the previous Sec. VA for the description of the pion emission (Fig. 6).

Equation (21) gives a contribution to the SL form factor where the initial and the final pion valence components appear [diagram (a) of Fig. 3]. The plus component of the quark-photon absorption vertex, given by $\Gamma^{+}\left(k^{\prime}-P_{\pi}, q\right)$ with $k^{\prime-}=k_{\text {on }}^{\prime-}$ which enters in Eq. (21), is represented by an empty circle in diagram (a) of Fig. 3 and is approximated by the sum of (i) the bare photon vertex multiplied by a renormalization constant, $a$ [diagram (a) of Fig. 7] and (ii) the contribution due to the $2 q 2 \bar{q}$ component of the final pion wave function, which is represented by diagram (b) of Fig. 7.

Therefore, we can make the following identification:

$$
\begin{aligned}
{\left[\left[\Gamma^{+}\left(k^{\prime}-P_{\pi}, q\right)\right]_{\left(k^{\prime-}=k_{\mathrm{on}}^{\prime-}\right)}\right]_{\alpha \beta}=} & a\left(\gamma^{+}\right)_{\alpha \beta}+\sum_{\alpha^{\prime} \beta^{\prime}} \int_{0}^{q^{+}} \frac{d k^{\prime \prime+} d \mathbf{k}_{\perp}^{\prime \prime}}{k^{\prime \prime+}\left(q^{+}-k^{\prime \prime+}\right)} \mathcal{H}_{\alpha \beta}^{\alpha^{\prime} \beta^{\prime}}\left(k^{\prime+}-P_{\pi}^{+}, \mathbf{k}_{\perp}^{\prime}-\mathbf{P}_{\pi \perp} ; k^{\prime \prime+}, \mathbf{k}_{\perp}^{\prime \prime} ; P_{\pi^{\prime}}^{-}, P_{\pi^{\prime}}^{+}, \mathbf{P}_{\pi^{\prime} \perp}\right) \\
& \times\left[\psi_{\gamma}^{+}\left(k^{\prime \prime+}, \mathbf{k}_{\perp}^{\prime \prime} ; q^{-}, q^{+}, \mathbf{q}_{\perp}\right)\right]_{\alpha^{\prime} \beta^{\prime} .}
\end{aligned}
$$

As already discussed in Sec. II, we do not consider the bare term photon vertex in the model of the present paper, since it violates current conservation for a massless pion (see Appendix C). Therefore, disregarding the bare photon vertex in the right-hand side of Eq. (41), we can formally write

$$
\left[\Gamma^{+}\left(k^{\prime}-P_{\pi}, q\right)\right]_{\left(k^{\prime-}=k_{\mathrm{on}}^{\prime-}\right.} \simeq \mathcal{H} \psi_{\gamma}^{+}
$$
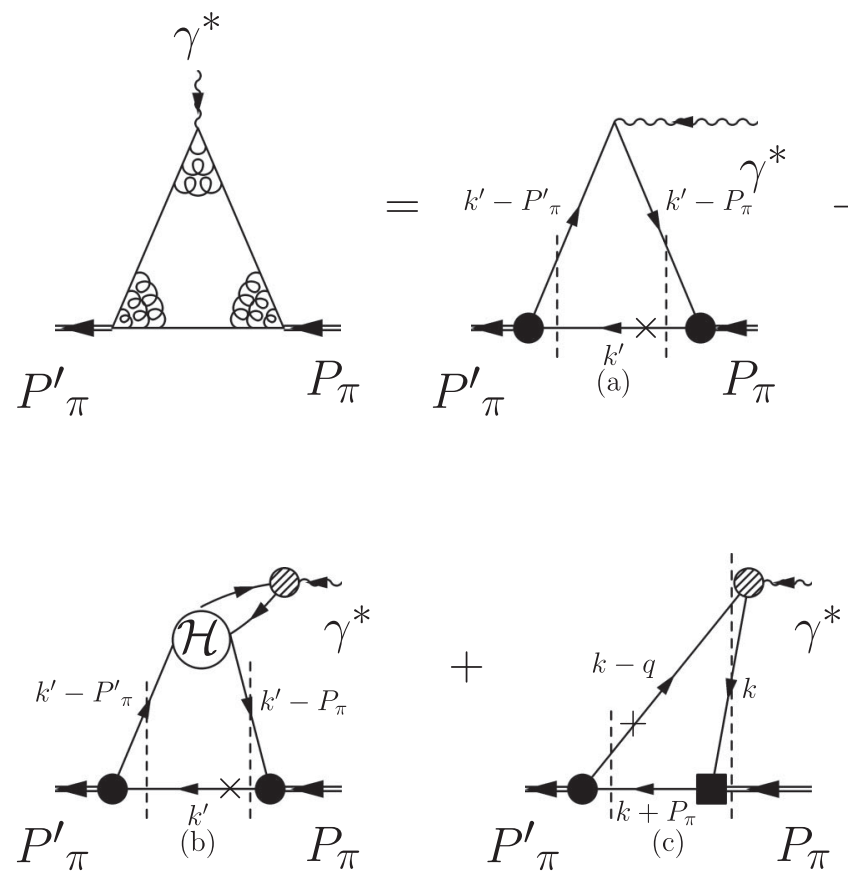

FIG. 7. Spacelike em form factor of the pion, $\pi \gamma^{*} \rightarrow \pi^{\prime}$, for $q^{+}>0$. Two possible light-front time orderings are shown. The first one allows to single out the following processes: (a) where a pointlike quark-photon interaction occurs, and (b) where the absorption of a $q \bar{q}$ pair by a quark proceeds through the kernel $\mathcal{H}$. Diagram (c) where the process $\gamma^{*} \rightarrow q \bar{q}$ appears (pairproduction process) with the subsequent absorption of the initial pion by a quark, corresponds to the second time ordering.
One could try to interpret Eq. (42) in terms of constituent quark form factors. However, we have to point out that the absorption vertex of Eq. (41) does not depend only on $q^{2}$, as one could naively think, but it depends on the virtuality of the quark as well.

Let us note that, within our assumption of a vanishing pion mass, the contribution of Eq. (21) is vanishing (see Sec. VIII) and therefore there is no contribution from $\left[\Gamma^{+}\left(k^{\prime}-P_{\pi}, q\right)\right]_{\left(k^{\prime-}=k_{\text {on }}^{\prime}\right)}$.

Equation (23) represents the pair-production term ( $Z$ diagram) and is depicted in Fig. 7(c) . The quark-pion absorption vertex, given by $\Lambda_{\pi}\left(-k ; P_{\pi}\right)$ evaluated at $k^{-}=$ $q^{-}+(k-q)_{\text {on }}^{-}$, which appears in Eq. (23) can be written as

$$
\begin{aligned}
\mathcal{D}_{\pi}:= & \frac{m}{f_{\pi}}\left[\Lambda_{\pi}\left(-k ; P_{\pi}\right)\right]_{\left(k^{-}=q^{-}+(k-q)_{\text {on }}^{-}\right)} \\
= & \frac{1}{4} \sum_{\alpha^{\prime} \beta^{\prime} \alpha \beta} \int_{0}^{P_{\pi}^{+}} \frac{d k^{\prime+} d \mathbf{k}_{\perp}^{\prime}}{k^{\prime+}\left(P_{\pi}^{+}-k^{\prime+}\right)}\left(\gamma^{5}\right)_{\beta \alpha}\left(\gamma^{5}\right)^{\beta^{\prime} \alpha^{\prime}} \\
& \times \mathcal{K}_{\alpha^{\prime} \beta^{\prime}}^{\alpha \beta}\left(k^{+}, \mathbf{k}_{\perp} ; k^{\prime+}, \mathbf{k}_{\perp}^{\prime} ; P_{\pi^{\prime}}^{-}, P_{\pi^{\prime}}^{+}, \mathbf{P}_{\pi^{\prime} \perp}\right) \\
& \times \psi_{\pi}\left(k^{\prime+}, \mathbf{k}_{\perp}^{\prime} ; P_{\pi}^{+}, \mathbf{P}_{\pi \perp}\right) .
\end{aligned}
$$

For our purpose this quark-pion absorption vertex will be taken constant, as we do in the TL case for the quark-pion emission vertex, as was proposed in Ref. [18] (see Sec. IX).

\section{TRIANGLE DIAGRAM AND PION LF WAVE FUNCTION}

\section{A. Timelike case}

Let us insert into Eq. (17) the photon vertex of Eq. (10). Furthermore, whenever the full expression for the lightfront pion wave function $\phi_{\pi}\left(k^{+}, \mathbf{k}_{\perp} ; P_{\pi}^{+}, \mathbf{P}_{\pi \perp}\right)$, given by Eq. (26), appears in Eq. (17) and the momentum fraction is in the valence-sector range $[0,1]$, let us replace it with the expression of Eq. (28), i.e. let us write the pion vertex in terms of the momentum component of the HLFD pion 
wave function. This means that we introduce in Eq. (17) the wave functions $\psi_{\pi}\left(k^{+}, \mathbf{k}_{\perp} ; P_{\pi}^{+}, \mathbf{P}_{\pi \perp}\right)$ and $\psi_{\bar{\pi}}\left(\left(k^{+}-\right.\right.$ $\left.\left.P_{\pi}^{+}\right),\left(\mathbf{k}-\mathbf{P}_{\pi}\right)_{\perp} ; P_{\bar{\pi}}^{+}, \mathbf{P}_{\bar{\pi} \perp}\right)$ when these wave functions have the correct support. Then the triangle diagram can be expressed as follows:

$$
\begin{aligned}
j^{\mu}= & \frac{e}{(2 \pi)^{3}} \frac{m}{f_{\pi}} N_{c} \int_{0}^{q^{+}} \frac{d k^{+} d \mathbf{k}_{\perp}}{\left(k^{+}-P_{\pi}^{+}\right) k^{+}\left(q^{+}-k^{+}\right)} \\
& \times \sum_{n, \lambda} \frac{\sqrt{2}\left[\epsilon_{\lambda}^{\mu}\left(P_{n}\right)\right]^{*} f_{V n}}{\left[q^{2}-M_{n}^{2}+\imath M_{n} \tilde{\Gamma}_{n}\left(q^{2}\right)\right]} \\
& \times\left\{\Theta\left(P_{\pi}^{+}-k^{+}\right) I_{1, n, \lambda}+\Theta\left(k^{+}-P_{\pi}^{+}\right) I_{2, n, \lambda}\right\} .
\end{aligned}
$$

The quantities $I_{1, n, \lambda}$ and $I_{2, n, \lambda}$ in Eq. (44) are defined as follows:

$$
\begin{aligned}
I_{1, n, \lambda}= & {\left[\Lambda_{\bar{\pi}}\left(k-P_{\pi}, P_{\bar{\pi}}\right) \Lambda_{n}\left(k, P_{n}\right)\right]_{k^{-}=k_{\mathrm{on}}^{-}} } \\
& \times\left\{\frac{q^{+}}{\left[q^{2}-M_{0}^{2}\left(k^{+}, \mathbf{k}_{\perp} ; q^{+}, \mathbf{q}_{\perp}\right)+\imath \epsilon\right]}\right. \\
& \left.\times\left[T_{\mathrm{on},(1, n, \lambda)}+T_{1,(1, n, \lambda)}\right]+T_{2,(1, n, \lambda)}\right\} \\
I_{2, n, \lambda}= & {\left[\bar{\Lambda}_{\pi}\left(k, P_{\pi}\right) \Lambda_{n}\left(k, P_{n}\right)\right]_{k^{-}=q^{-}(k-q)_{\mathrm{on}}^{-}} } \\
& \times\left\{\frac{q^{+}}{\left[q^{2}-M_{0}^{2}\left(k^{+}, \mathbf{k}_{\perp} ; q^{+}, \mathbf{q}_{\perp}\right)+\imath \epsilon\right]}\right. \\
& \left.\times\left[T_{\mathrm{on},(2, n, \lambda)}+T_{1,(2, n, \lambda)}\right]+T_{3,(2, n, \lambda)}\right\}
\end{aligned}
$$

where

$$
\begin{aligned}
T_{\mathrm{on},(1, n, \lambda)}= & \psi_{\pi}^{*}\left(k^{+}, \mathbf{k}_{\perp} ; P_{\pi}^{+}, \mathbf{P}_{\pi \perp}\right) \operatorname{Tr}\left[\left[\left(\not k-\not P_{\pi}\right)_{\mathrm{on}}+m\right]\right. \\
& \times \gamma^{5}\left[(k-\not)_{\mathrm{on}}+m\right] \\
& \left.\times\left[\epsilon_{\lambda}\left(P_{n}\right) \cdot \hat{V}_{n}\left(k, k-P_{n}\right)\right]_{k^{-}=k_{\mathrm{on}}^{-}}\left(k_{\mathrm{on}}+m\right) \gamma^{5}\right],
\end{aligned}
$$

$$
\begin{aligned}
T_{\mathrm{on},(2, n, \lambda)}= & -\psi_{\bar{\pi}}^{*}\left(\left(k^{+}-P_{\pi}^{+}\right),\left(\mathbf{k}-\mathbf{P}_{\pi}\right)_{\perp} ; P_{\bar{\pi}}^{+}, \mathbf{P}_{\bar{\pi} \perp}\right) \\
& \times \operatorname{Tr}\left[\left(\not k-\not P_{\pi}\right)_{\mathrm{on}}+m\right] \gamma^{5}\left[(k-\not)_{\mathrm{on}}+m\right] \\
& \times\left[\epsilon_{\lambda}\left(P_{n}\right) \cdot \hat{V}_{n}\left(k, k-P_{n}\right)\right]_{k^{-}=q^{-}+(k-q)_{\mathrm{on}}^{-}} \\
& \left.\times\left(k_{\mathrm{on}}+m\right) \gamma^{5}\right],
\end{aligned}
$$

$$
\begin{aligned}
T_{1,(1, n, \lambda)}= & -\frac{1}{2} \frac{m}{f_{\pi}}\left[\bar{\Lambda}_{\pi}\left(k ; P_{\pi}\right)\right]_{k^{-}=k_{\mathrm{on}}^{-}} \\
& \times \operatorname{Tr}\left[\gamma^{+} \gamma^{5}\left[(k-\not)_{\mathrm{on}}+m\right]\right. \\
& \left.\times\left[\epsilon_{\lambda}\left(P_{n}\right) \cdot \hat{V}_{n}\left(k, k-P_{n}\right)\right]_{k^{-}=k_{\mathrm{on}}^{-}}\left(k_{\mathrm{on}}+m\right) \gamma^{5}\right],
\end{aligned}
$$

$$
\begin{aligned}
T_{1,(2, n, \lambda)}= & -\frac{1}{2} \frac{m}{f_{\pi}}\left[\Lambda_{\bar{\pi}}\left(k-P_{\pi}, P_{\bar{\pi}}\right)\right]_{k^{-}=q^{-}+(k-q)_{\mathrm{on}}^{-}} \\
& \times \operatorname{Tr}\left[\gamma^{+} \gamma^{5}\left[(k-\not)_{\mathrm{on}}+m\right]\right. \\
& \times\left[\epsilon_{\lambda}\left(P_{n}\right) \cdot \hat{V}_{n}\left(k, k-P_{n}\right)\right]_{k^{-}=q^{-}+(k-q)_{\mathrm{on}}^{-}} \\
& \left.\times\left(k_{\mathrm{on}}+m\right) \gamma^{5}\right], \\
T_{2,(1, n, \lambda)}= & -\frac{1}{2} \psi_{\pi}^{*}\left(k^{+}, \mathbf{k}_{\perp} ; P_{\pi}^{+}, \mathbf{P}_{\pi \perp}\right) \\
& \times \operatorname{Tr}\left[[ ( k - \not P _ { \pi } ) _ { \mathrm { on } } + m ] \gamma ^ { 5 } \gamma ^ { + } \left[\epsilon_{\lambda}\left(P_{n}\right)\right.\right. \\
& \left.\left.\cdot \hat{V}_{n}\left(k, k-P_{n}\right)\right]_{k^{-}}=k_{\mathrm{on}}^{-}\left(k_{\mathrm{on}}+m\right) \gamma^{5}\right],
\end{aligned}
$$

$$
\begin{aligned}
T_{3,(2, n, \lambda)}= & -\frac{1}{2} \psi_{\bar{\pi}}^{*}\left(\left(k^{+}-P_{\pi}^{+}\right),\left(\mathbf{k}-\mathbf{P}_{\pi}\right)_{\perp} ; P_{\bar{\pi}}^{+}, \mathbf{P}_{\bar{\pi} \perp}\right) \\
& \times \operatorname{Tr}\left[\left[\left(k-\not P_{\pi}\right)_{\mathrm{on}}+m\right] \gamma^{5}\left[(k-\not)_{\mathrm{on}}+m\right]\right. \\
& \left.\times\left[\epsilon_{\lambda}\left(P_{n}\right) \cdot \hat{V}_{n}\left(k, k-P_{n}\right)\right]_{k^{-}=q^{-}+(k-q)_{\mathrm{on}}^{-}} \gamma^{+} \gamma^{5}\right]
\end{aligned}
$$

Let us notice that the momentum component of the LF pion wave function does not appear in the instantaneous terms $T_{1,(1, n, \lambda)}$ and $T_{1,(2, n, \lambda)}$, because in these terms the propagator $\left[\left(\not k-\not P_{\pi}\right)_{\text {on }}+m\right] /\left[k^{-}-P_{\pi}^{-}-\left(k-P_{\pi}\right)_{\text {on }}^{-}+\imath \epsilon /\left(k^{+}-\right.\right.$ $\left.\left.P_{\pi}^{+}\right)\right]$is replaced by $\gamma^{+} / 2$. Indeed the amplitude

$$
\frac{1}{2} \frac{m}{f_{\pi}}\left(k_{\mathrm{on}}+m\right) \gamma^{5}\left[\bar{\Lambda}_{\pi}\left(k ; P_{\pi}\right)\right]_{k^{-}=k_{\mathrm{on}}^{-}} \gamma^{+}
$$

does not obey the same two-body Schrödinger-like equation as the light-front pion wave function $\phi_{\pi}\left(k^{+}, \vec{k}_{\perp} ; P_{\pi}^{+}, \vec{P}_{\pi \perp}\right)$ does.

As already noted at the end of Sec. III A, the first and the second term of Eq. (44) are represented in Fig. 2 by the diagrams (a) and (b), respectively. Note that, due to the $\Theta$ functions, the final pion or antipion wave functions enter into the first or the second term of Eq. (44), respectively. In Eq. (44) the pion vertices $\left[\Lambda_{\bar{\pi}}\left(k-P_{\pi} ; P_{\bar{\pi}}\right)\right]$, evaluated at $k^{-}=k_{\mathrm{on}}^{-}$, and $\left[\bar{\Lambda}_{\pi}\left(k ; P_{\pi}\right)\right]$, evaluated at $k^{-}=q^{-}+(k-$ $q)_{\text {on }}^{-}$, have the momentum fraction outside the valencesector range $[0,1]$ and can be related to the quark amplitudes for radiative antipion or pion emission, respectively [see Figs. 2(a) and 2(b)]. The presence of these vertices gives rise to the contribution of the nonvalence component of the virtual-photon wave function, relevant for the process under consideration. In the spacelike region the analogous processes can be interpreted for $q^{+}>0$ as the contribution of the nonvalence component of the pion wave function in the final state [18]. These points have already been illustrated in Sec. V.

If we choose to take the Dirac structures in the photon vertex with both quarks on their mass shell, i.e. $\Gamma^{\mu}(k, q)=$ $\left[\Gamma^{\mu}(k, q)\right]_{\text {on }}$ [see Eq. (37)], then whenever the full expres- 
sion for the light-front vector-meson wave function $\phi_{n \lambda}\left(k^{+}, \vec{k}_{\perp} ; P_{n}^{+}, \vec{P}_{n \perp}\right)$, given by Eq. (31), appears in Eq. (44), we can take advantage of our identification of Eqs. (31) and (32) to express the quantities $I_{1, n, \lambda}$ and $I_{2, n, \lambda}$ through the momentum component of the HLFD VM wave function. However, in the instantaneous contributions to $I_{1, n, \lambda}$ and $I_{2, n, \lambda}$ which are proportional to the quantities
$T_{2,(1, n, \lambda)}$ and $T_{3,(2, n, \lambda)}$ we do not express the VM vertex functions $\left[\Lambda_{n}\left(k, P_{n}\right)\right]_{k^{-}=k_{\mathrm{on}}^{-}}$and $\left[\Lambda_{n}\left(k, P_{n}\right)\right]_{k^{-}=q^{-}+(k-q)_{\text {on }}^{-}}$ through the momentum component of the HLFD VM wave function, because the full expression for this function given by Eq. (32) does not appear in these instantaneous terms.

Then we obtain

$$
\begin{aligned}
I_{1, n, \lambda}= & {\left[\Lambda_{\bar{\pi}}\left(k-P_{\pi}, P_{\bar{\pi}}\right)\right]_{k^{-}=k_{\mathrm{on}}^{-}}\left\{\frac{\psi_{n}\left(k^{+}, \mathbf{k}_{\perp} ; P_{n}^{+}, \mathbf{P}_{n \perp}\right)\left[M_{n}^{2}-M_{0}^{2}\left(k^{+}, \mathbf{k}_{\perp} ; P_{n}^{+}, \mathbf{P}_{n \perp}\right)\right]}{\left[q^{2}-M_{0}^{2}\left(k^{+}, \mathbf{k}_{\perp} ; q^{+}, \mathbf{q}_{\perp}\right)+\imath \epsilon\right]}\left[T_{\mathrm{on},(1, n, \lambda)}+T_{1,(1, n, \lambda)}\right]\right.} \\
& \left.+\left[\Lambda_{n}\left(k, P_{n}\right)\right]_{k^{-}=k_{\mathrm{on}}^{-}} T_{2,(1, n, \lambda)}\right\}, \\
I_{2, n, \lambda}= & {\left[\bar{\Lambda}_{\pi}\left(k, P_{\pi}\right)\right]_{k^{-}=q^{-}+(k-q)_{\mathrm{on}}^{-}}\left\{\frac{\psi_{n}\left(k^{+}, \mathbf{k}_{\perp} ; P_{n}^{+}, \mathbf{P}_{n \perp}\right)\left[M_{n}^{2}-M_{0}^{2}\left(k^{+}, \mathbf{k}_{\perp} ; P_{n}^{+}, \mathbf{P}_{n \perp}\right)\right]}{\left[q^{2}-M_{0}^{2}\left(k^{+}, \mathbf{k}_{\perp} ; q^{+}, \mathbf{q}_{\perp}\right)+\imath \epsilon\right]}\left[T_{\mathrm{on},(2, n, \lambda)}+T_{1,(2, n, \lambda)}\right]\right.} \\
& \left.+\left[\Lambda_{n}\left(k, P_{n}\right)\right]_{k^{-}=q^{-}+(k-q)_{\mathrm{on}}^{-}} T_{3,(2, n, \lambda)}\right\} .
\end{aligned}
$$

The quantities $T_{\text {on, }(1, n, \lambda)}, T_{1,(1, n, \lambda)}, T_{2,(1, n, \lambda)}$ and the quantities $T_{\text {on, }(2, n, \lambda)}, T_{1,(2, n, \lambda)}, T_{3,(2, n, \lambda)}$ in Eqs. (54) and (55) have the same expressions as in Eqs. (47), (49), (51), (48), (50), and (52), respectively, with $\left[\epsilon_{\lambda}\left(P_{n}\right) \cdot \hat{V}_{n}\left(k, k-P_{n}\right)\right]_{k^{-}=k_{\text {on }}^{-}}$ and $\left[\epsilon_{\lambda}\left(P_{n}\right) \cdot \hat{V}_{n}\left(k, k-P_{n}\right)\right]_{k^{-}=q^{-}+(k-q)}$ - both replaced by $\epsilon_{\lambda}\left(P_{n}\right) \cdot\left[\hat{V}_{n}\left(k, k-P_{n}\right)\right]_{\text {on }}$ [see Eq. (16) for the definition of $\left.\left[\hat{V}_{n}\left(k, k-P_{n}\right)\right]_{\text {on }}\right]$.

Note that the region of integration over $k^{+}$in Eq. (44) (a consequence of the nonvanishing integration in the $k^{-}$ complex plane) agrees with the support of the wave functions $\psi_{\pi}$ and $\psi_{\bar{\pi}}$ of Eqs. (47) and (48), respectively. Furthermore, in agreement with the above assumptions, the vertex associated with the virtual photon and consequently the wave function $\psi_{n}\left(k^{+}, \mathbf{k}_{\perp} ; P_{n}^{+}, \mathbf{P}_{n \perp}\right)$ in Eqs. (54) and (55) have the intrinsic fraction of the plus component of the quark momentum, $k^{+} / q^{+}=k^{+} / P_{n}^{+}$, in the interval $[0,1]$.

To be able to evaluate the TL pion form factor we have still to assign a value, in the instantaneous terms, to the VM vertex functions $\left[\Lambda_{n}\left(k, P_{n}\right)\right]_{k^{-}=k_{\text {on }}^{-}}$and
$\left[\Lambda_{n}\left(k, P_{n}\right)\right]_{k^{-}=q^{-}+(k-q)_{\text {on }}^{-}}$, as well as to the pion vertex functions $\left[\bar{\Lambda}_{\pi}\left(k ; P_{\pi}\right)\right]_{k^{-}=k_{\text {on }}^{-}}$and $\left[\Lambda_{\bar{\pi}}(k-\right.$ $\left.\left.P_{\pi}, P_{\bar{\pi}}\right)\right]_{k^{-}=q^{-}+(k-q)_{\text {on }}^{-}}$.

\section{B. Spacelike case}

As in the timelike case, let us replace in Eqs. (21) and (23) the pion vertex function with its expression in terms of the momentum component of the HLFD pion wave function, whenever the full expression for the LF pion wave function [Eq. (26)] appears, taking advantage of our identification of Eqs. (26) and (28).

\section{Valence region contribution}

Substituting in Eq. (21) the pion initial and final wave functions and noting that for the final pion the bar vertex gives the complex conjugate wave function, while in the initial state the vertex gives the initial pion wave function, in the valence region one gets (see Fig. 3):

$$
\begin{aligned}
j^{(\mathrm{I}) \mu}= & \frac{e}{(2 \pi)^{3}} N_{c} \int_{0}^{P_{\pi}^{+}} \frac{d k^{\prime+} d \mathbf{k}_{\perp}^{\prime}}{\left(k^{\prime+}-P_{\pi}^{+}\right) k^{\prime+}\left(P_{\pi^{\prime}}^{+}-k^{\prime+}\right)}\left[\bar{T}_{\mathrm{on},(4)}^{\mu} \psi_{\pi^{\prime}}^{*}\left(k^{\prime+}, \mathbf{k}_{\perp}^{\prime} ; P_{\pi^{\prime}}^{+}, \mathbf{P}_{\pi^{\prime} \perp}\right) \psi_{\pi}\left(P_{\pi}^{+}-k^{\prime+}, \mathbf{P}_{\pi \perp}-\mathbf{k}_{\perp}^{\prime} ; P_{\pi}^{+}, \mathbf{P}_{\pi \perp}\right)\right. \\
& +\bar{T}_{2,(4)}^{\mu} \psi_{\pi}\left(P_{\pi}^{+}-k^{\prime+}, \mathbf{P}_{\pi \perp}-\mathbf{k}_{\perp}^{\prime} ; P_{\pi}^{+}, \mathbf{P}_{\pi \perp}\right)\left[\bar{\Lambda}_{\pi^{\prime}}\left(k^{\prime}, P_{\pi^{\prime}}\right)\right]_{k^{\prime}=k_{\mathrm{on}}^{\prime-}}+\bar{T}_{3,(4)}^{\mu} \psi_{\pi^{\prime}}^{*}\left(k^{\prime+}, \mathbf{k}_{\perp}^{\prime} ; P_{\pi^{\prime}}^{+}, \mathbf{P}_{\pi^{\prime} \perp}\right) \\
& \left.\times\left[\Lambda_{\pi}\left(P_{\pi}-k^{\prime}, P_{\pi}\right)\right]_{k^{\prime-}=k_{\mathrm{on}}^{\prime}}\right]
\end{aligned}
$$

where

$$
\begin{gathered}
\bar{T}_{o n,(4)}^{\mu}=\operatorname{Tr}\left[\left(\not k_{\mathrm{on}}^{\prime \prime}+m\right) \gamma^{5}\left[\left(\not k^{\prime}-\not \phi_{\pi^{\prime}}\right)_{\mathrm{on}}+m\right] \Gamma^{\mu}(4)\left[\left(\not k^{\prime}-\not \not_{\pi}\right)_{\mathrm{on}}+m\right] \gamma^{5}\right] \\
\bar{T}_{2,(4)}^{\mu}=-\frac{1}{2} \operatorname{Tr}\left[\left(\not k_{\mathrm{on}}^{\prime}+m\right) \gamma^{5} \gamma^{+} \Gamma^{\mu}(4)\left[\left(\not k^{\prime}-\not \boldsymbol{P}_{\pi}\right)_{\mathrm{on}}+m\right] \gamma^{5}\right]
\end{gathered}
$$




$$
\bar{T}_{3,(4)}^{\mu}=-\frac{1}{2} \operatorname{Tr}\left[\left(k_{\mathrm{on}}^{\prime}+m\right) \gamma^{5}\left[\left(\mathcal{k}^{\prime}-\not{ }_{\pi^{\prime}}\right)_{\mathrm{on}}+m\right] \Gamma^{\mu}(4) \gamma^{+} \gamma^{5}\right]
$$

In the instantaneous terms proportional to $\bar{T}_{2,(4)}^{\mu}$ and to $\bar{T}_{3(4)}^{\mu}$ we do not express the pion vertex functions $\left[\Lambda_{\pi^{\prime}}^{(4)}\left(k^{\prime}, P_{\pi^{\prime}}\right)\right]_{k^{\prime-}=k_{\text {on }}^{\prime-}}$ and $\left[\Lambda_{\pi}\left(P_{\pi}-k^{\prime}, P_{\pi}\right)\right]_{k^{\prime-}=k_{\text {on }}^{\prime}}$ in terms of the momentum component of the HLFD pion wave function, because of the presence of $\gamma^{+}$instead of $\left[\left(k^{\prime}-\right.\right.$ $\left.\left.\not \bar{\pi}^{\prime}\right)_{\text {on }}+m\right]$ or $\left[\left(\mathbb{k}^{\prime}-\not p_{\pi}\right)_{\text {on }}+m\right]$, respectively.

In Eq. (56) the photon vertex, $\Gamma^{\mu}(4)=\Gamma^{\mu}\left(k^{\prime}-P_{\pi}, q\right)$, evaluated at $k^{\prime-}=k_{\mathrm{on}}^{\prime-}$, is the amplitude for the photon absorption by a quark. As discussed in Sec. V B, the photon absorption operator can be decomposed in a bare vertex, i.e. $\gamma^{\mu}$ [Fig. 7(a)], plus other terms. From the expansion in the light-front Fock space, the next term relevant for the process we are analyzing is due to a contribution of the nonvalence $2 q 2 \bar{q}$ component of the final pion wave function; see diagram (b) of Fig. 7. This contribution can be thought of as an expectation value of an operator between the valence component of the wave functions for the initial and final pions. The operator can be constructed by applying to the virtual-photon wave function the kernel, $\mathcal{H}$, which produces the nonvalence pion component from the valence one [see Eq. (41)].

In Sec. VIII, it will be shown, assuming a massless pion, that $P_{\pi}^{+}=0$, and therefore $j^{(\mathrm{I}) \mu}$ vanishes.

\section{Pair-production contribution}

Also the pair-production contribution to the current can be rewritten in terms of the momentum component of the HLFD pion wave function [see Eqs. (26) and (28)] when the light-front pion wave function appears. Then Eq. (23) becomes [see Fig. 3(b)]:

$$
\begin{aligned}
j^{(\mathrm{II}) \mu}= & -\frac{e N_{c}}{(2 \pi)^{3}} \frac{m}{f_{\pi}} \int_{0}^{q^{+}} \frac{d k^{+} d \mathbf{k}_{\perp}\left[\Lambda_{\pi}\left(-k ; P_{\pi}\right)\right]_{k^{-}=q^{-}+(k-q)_{\mathrm{on}}^{-}}}{\left(k^{+}+P_{\pi}^{+}\right) k^{+}\left(q^{+}-k^{+}\right)} \frac{\sqrt{2}\left[\epsilon_{\lambda}^{\mu}\left(P_{n}\right)\right]^{*} f_{V n}}{\left[q^{2}-M_{n}^{2}+\imath M_{n} \tilde{\Gamma}_{n}\left(q^{2}\right)\right]}\left[\Lambda_{n}\left(k, P_{n}\right)\right]_{k^{-}=q^{-}+(k-q)_{\mathrm{on}}^{-}} \\
& \times\left\{\frac{q^{+}}{\left[q^{2}-M_{0}^{2}\left(k^{+}, \mathbf{k}_{\perp} ; q^{+}, \mathbf{q}_{\perp}\right)+\imath \epsilon\right]}\left[T_{\mathrm{on},(2, n)}^{\prime}+T_{1,(2, n)}^{\prime}\right]+T_{3,(2, n)}^{\prime}\right\},
\end{aligned}
$$

where

$$
\begin{aligned}
T_{\mathrm{on},(2, n)}^{\prime}= & \psi_{\pi^{\prime}}^{*}\left(\left(k^{+}+P_{\pi}^{+}\right),\left(\mathbf{k}+\mathbf{P}_{\pi}\right)_{\perp} ; P_{\pi^{\prime}}^{+}, \mathbf{P}_{\pi^{\prime} \perp}\right) \operatorname{Tr}\left[\left[\left(k+\not P_{\pi}\right)_{\mathrm{on}}+m\right] \gamma^{5}\left[(k-\not k)_{\mathrm{on}}+m\right]\right. \\
& \left.\times\left[\epsilon_{\lambda}\left(P_{n}\right) \cdot \hat{V}_{n}\left(k, k-P_{n}\right)\right]_{k^{-}=q^{-}+(k-q)_{\mathrm{on}}^{-}}\left(k_{\mathrm{on}}+m\right) \gamma^{5}\right], \\
T_{1,(2, n)}^{\prime}= & \frac{1}{2} \frac{m}{f_{\pi}}\left[\bar{\Lambda}_{\pi^{\prime}}\left(k+P_{\pi}, P_{\pi^{\prime}}\right)\right]_{k^{-}=q^{-}+(k-q)_{\mathrm{on}}^{-}} \operatorname{Tr}\left[\gamma^{+} \gamma^{5}\left[(k-\not)_{\mathrm{on}}+m\right]\right. \\
& \left.\times\left[\epsilon_{\lambda}\left(P_{n}\right) \cdot \hat{V}_{n}\left(k, k-P_{n}\right)\right]_{k^{-}=q^{-}+(k-q)_{\mathrm{on}}^{-}}\left(\not k_{\mathrm{on}}+m\right) \gamma^{5}\right], \\
T_{3,(2, n)}^{\prime}= & \frac{1}{2} \psi_{\pi^{\prime}}^{*}\left(\left(k^{+}+P_{\pi}^{+}\right),\left(\mathbf{k}+\mathbf{P}_{\pi}\right)_{\perp} ; P_{\pi^{\prime}}^{+}, \mathbf{P}_{\pi^{\prime} \perp}\right) \operatorname{Tr}\left[\left[\left(k+\not P_{\pi}\right)_{\mathrm{on}}+m\right] \gamma^{5}\left[(k-\not)_{\mathrm{on}}+m\right]\right. \\
& \left.\times\left[\epsilon_{\lambda}\left(P_{n}\right) \cdot \hat{V}_{n}\left(k, k-P_{n}\right)\right]_{k^{-}=q^{-}+(k-q)_{\mathrm{on}}^{-}} \gamma^{+} \gamma^{5}\right] .
\end{aligned}
$$

As for the timelike case, we have used the expression of Eq. (10) for the photon vertex with the virtual photon going into a $q \bar{q}$ pair. The bar vertex $\bar{\Lambda}_{\pi^{\prime}}$ implies that the final pion wave function in the above expressions has to be complex conjugated. If we take the Dirac structures in the photon vertex with both the quarks on their mass shell, as in the timelike case [see Eq. (37)], then using Eqs. (31) and (32) we can express $j^{(\mathrm{II}) \mu}$ through the momentum component of the HLFD vector-meson wave functions, when the LF VM wave function is present, i.e. in the terms given by Eqs. (61) and (62):

$$
\begin{aligned}
j^{(\mathrm{II}) \mu}= & -\frac{e N_{c}}{(2 \pi)^{3}} \frac{m}{f_{\pi}} \int_{0}^{q^{+}} \frac{d k^{+} d \mathbf{k}_{\perp}\left[\Lambda_{\pi}\left(-k ; P_{\pi}\right)\right]_{k^{-}=q^{-}+(k-q)_{\mathrm{on}}^{-}}}{\left(k^{+}+P_{\pi}^{+}\right) k^{+}\left(q^{+}-k^{+}\right)} \sum_{n, \lambda} \frac{\sqrt{2}\left[\epsilon_{\lambda}^{\mu}\left(P_{n}\right)\right]^{*} f_{V n}}{\left[q^{2}-M_{n}^{2}+\imath M_{n} \tilde{\Gamma}_{n}\left(q^{2}\right)\right]} \\
& \times\left\{\frac{\psi_{n}\left(k^{+}, \mathbf{k}_{\perp} ; P_{n}^{+}, \mathbf{P}_{n \perp}\right)\left[M_{n}^{2}-M_{0}^{2}\left(k^{+}, \mathbf{k}_{\perp} ; P_{n}^{+}, \mathbf{P}_{n \perp}\right)\right]}{\left[q^{2}-M_{0}^{2}\left(k^{+}, \mathbf{k}_{\perp} ; q^{+}, \mathbf{q}_{\perp}\right)+\imath \epsilon\right]}\left[T_{\mathrm{on},(2, n)}^{\prime}+T_{1,(2, n)}^{\prime}\right]+\left[\Lambda_{n}\left(k, P_{n}\right)\right]_{k^{-}=q^{-}+(k-q)_{\mathrm{on}}^{-}} T_{3,(2, n)}^{\prime}\right\},
\end{aligned}
$$

with 


$$
\begin{aligned}
& T_{\mathrm{on},(2, n)}^{\prime}=\psi_{\pi \prime}^{*}\left(\left(k^{+}+P_{\pi}^{+}\right),\left(\mathbf{k}+\mathbf{P}_{\pi}\right)_{\perp} ; P_{\pi^{\prime}}^{+}, \mathbf{P}_{\pi^{\prime} \perp}\right) \operatorname{Tr}\left[\left[\left(k+\not P_{\pi}\right)_{\mathrm{on}}+m\right] \gamma^{5}\left[(k-\not)_{\mathrm{on}}+m\right] \epsilon_{\lambda}\left(P_{n}\right)\right. \\
& \left.\cdot\left[\hat{V}_{n}\left(k, k-P_{n}\right)\right]_{\mathrm{on}}\left(k_{\mathrm{on}}+m\right) \gamma^{5}\right], \\
& T_{1,(2, n)}^{\prime}=\frac{1}{2} \frac{m}{f_{\pi}}\left[\bar{\Lambda}_{\pi^{\prime}}\left(k+P_{\pi}, P_{\pi \prime}\right)\right]_{k^{-}=q^{-}+(k-q)_{\mathrm{on}}^{-}} \operatorname{Tr}\left[\gamma^{+} \gamma^{5}\left[(k-\not)_{\mathrm{on}}+m\right] \epsilon_{\lambda}\left(P_{n}\right) \cdot\left[\hat{V}_{n}\left(k, k-P_{n}\right)\right]_{\mathrm{on}}\left(k_{\mathrm{on}}+m\right) \gamma^{5}\right], \\
& T_{3,(2, n)}^{\prime}=\frac{1}{2} \psi_{\pi^{\prime}}^{*}\left(\left(k^{+}+P_{\pi}^{+}\right),\left(\mathbf{k}+\mathbf{P}_{\pi}\right)_{\perp} ; P_{\pi^{\prime}}^{+}, \mathbf{P}_{\pi^{\prime} \perp}\right) \operatorname{Tr}\left[\left[\left(k+\not P_{\pi}\right)_{\mathrm{on}}+m\right] \gamma^{5}\left[(k-\not k)_{\mathrm{on}}+m\right] \epsilon_{\lambda}\left(P_{n}\right)\right. \\
& \left.\cdot\left[\hat{V}_{n}\left(k, k-P_{n}\right)\right]_{\mathrm{on}} \gamma^{+} \gamma^{5}\right] .
\end{aligned}
$$

The vertex $\Lambda_{\pi}\left(-k ; P_{\pi}\right)$ evaluated at $k^{-}=q^{-}+(k-$ $q)_{\text {on }}^{-}$represents the pion absorption amplitude by an onshell quark. The presence of this process can be also interpreted as a $2 q 2 \bar{q}$ component in the final pion wave function [see Figs. 3(b) and 7(c)], as illustrated in Sec. V.

\section{TIMELIKE EM FORM FACTOR OF THE PION}

We have pointed out in Sec. I that, for a unified description of TL and SL form factors, it is necessary to choose a reference frame where the plus component of the momentum transfer $q^{+}$is different from zero (otherwise, $q^{2}=$ $q^{+} q^{-}-\mathbf{q}_{\perp}^{2}$ cannot be positive). Therefore, as in Ref. [29], in order to calculate the pion form factor we adopt a reference frame where $\mathbf{q}_{\perp}=0$ and $q^{+}>0$.

The decay of a timelike virtual photon is written in terms of the timelike form factor of the pion as follows:

$$
j^{\mu}=\left\langle\pi \bar{\pi}\left|\bar{q}(0) \gamma^{\mu} q(0)\right| 0\right\rangle=e\left(P_{\pi}^{\mu}-P_{\bar{\pi}}^{\mu}\right) F_{\pi}\left(q^{2}\right),
$$

where $q^{\mu}=P_{\pi}^{\mu}+P_{\bar{\pi}}^{\mu}$ is the four-momentum of the virtual photon. In Fig. 2, the diagrammatic analysis of the virtualphoton decay in a $\pi \bar{\pi}$ pair is shown.

The virtual-photon decay amplitude can be obtained from Eq. (68) by evaluating the plus component of the matrix element $j^{\mu}$. To be able to evaluate the matrix element $j^{+}$from Eq. (44), we substitute in Eq. (44) constant values for the vertices, $\overline{\mathcal{D}}_{\pi}$ and $\mathcal{D}_{\bar{\pi}}$, namely, for pion or antipion radiation by a quark, Eqs. (39) and (40), respectively [see also Eqs. (45) and (46)]. Then, it remains to specify the values of the instantaneous vertex functions $\left[\bar{\Lambda}_{\pi}\left(k ; P_{\pi}\right)\right]_{k^{-}=k_{\mathrm{on}}^{-}}$in Eq. (49), $\quad\left[\Lambda_{\bar{\pi}}(k-\right.$ $\left.\left.P_{\pi}, P_{\bar{\pi}}\right)\right]_{k^{-}=q^{-}+(k-q)_{\text {on }}^{-}}$in Eq. (50), and $\left[\Lambda_{n}\left(k, P_{n}\right)\right]$ in Eqs. (54) and (55). This will be thoroughly discussed in Sec. IX.

By using Eqs. (68) and (44) one can obtain the pion form factor $F_{\pi}\left(q^{2}\right)$ from the plus component of the current:

$$
F_{\pi}\left(q^{2}\right)=\sum_{n} \frac{f_{V n}}{\left[q^{2}-M_{n}^{2}+\imath M_{n} \tilde{\Gamma}_{n}\left(q^{2}\right)\right]} g_{V n}^{+}\left(q^{2}\right)
$$

where $g_{V n}^{+}\left(q^{2}\right)$, for $q^{2}>0$, is the form factor for the VM decay in a pair of pions, as expected from the VMD approximation. The characteristic feature of our approach is that we aim at a microscopic description of both $f_{V n}$ and $g_{V n}^{+}\left(q^{2}\right)$.

In Appendix G we evaluate $g_{V n}^{+}\left(q^{2}\right)$ in the rest reference frame of the $n$ th-resonance, where $\mathbf{q}_{\perp}=0$ and $q^{+}=M_{n}$, taking advantage of the invariance of each term of $\sum_{n}$ in Eq. (69) under LF boosts. In the limit of a vanishing pion mass and making the purely longitudinal choice for the pion momentum, i.e. $\mathbf{P}_{\bar{\pi} \perp}=-\mathbf{P}_{\pi \perp}=\mathbf{0}$, one obtains

$$
g_{V n}^{+}\left(q^{2}\right)=\frac{1}{M_{n}} \frac{N_{c}}{(2 \pi)^{3}} \int_{0}^{q^{+}} \frac{\sqrt{2} d k^{+} d \mathbf{k}_{\perp}}{k^{+} k^{+}\left(q^{+}-k^{+}\right)} \bar{I}_{2, n},
$$

with

$$
\begin{aligned}
\bar{I}_{2, n}= & \overline{\mathcal{D}}_{\pi}\left\{\frac{\psi_{n}\left(k^{+}, \mathbf{k}_{\perp} ; P_{n}^{+}, \mathbf{0}\right)\left[M_{n}^{2}-M_{0}^{2}\left(k^{+}, \mathbf{k}_{\perp} ; P_{n}^{+}, \mathbf{0}\right)\right]}{\left[q^{2}-M_{0}^{2}\left(k^{+}, \mathbf{k}_{\perp} ; q^{+}, \mathbf{0}\right)+\imath \boldsymbol{\epsilon}\right]}\right. \\
& \left.\times \mathcal{T}_{1,(2, n)}+\left[\Lambda_{n}\left(k, P_{n}\right)\right]_{k^{-}=q^{-}+(k-q)_{\text {on }}^{-}} \mathcal{T}_{3,(2, n)}\right\} .
\end{aligned}
$$

The explicit expression for $\mathcal{T}_{1,(2, n)}$ and $\mathcal{T}_{3,(2, n)}$ can be found in Eqs. (G19) and (G20), respectively.

To evaluate the timelike pion form factor we have still to specify the values of the instantaneous vertex functions $\left[\Lambda_{\bar{\pi}}\left(k-P_{\pi}, P_{\bar{\pi}}\right)\right]_{k^{-}=q^{-}+(k-q)_{\text {on }}^{-}}$in Eq. (G19) and $\left[\Lambda_{n}\left(k, P_{n}\right)\right]_{k^{-}=q^{-}+(k-q)_{\text {on }}^{-}}$in Eq. (71) that, as already explained, cannot be directly related to $\psi_{\bar{\pi}}$ and $\psi_{n}$.

\section{SPACELIKE EM FORM FACTOR OF THE PION}

The spacelike form factor of the pion can be obtained from the plus component of the proper current matrix element

$$
j^{\mu}=\left\langle\pi\left|\bar{q}(0) \gamma^{\mu} q(0)\right| \pi^{\prime}\right\rangle=e\left(P_{\pi}^{\mu}+P_{\pi^{\prime}}^{\mu}\right) F_{\pi}\left(q^{2}\right),
$$

where $q^{\mu}=P_{\pi^{\prime}}^{\mu}-P_{\pi}^{\mu}$.

In our reference frame, where $\mathbf{q}_{\perp}=0$ and $q^{+}>0$, the minus component of the four-momentum transfer is given by $q^{-}=q^{2} / q^{+}$, which is negative in the spacelike region. Let us note that

$$
q^{-}=\frac{\left|\mathbf{P}_{\pi^{\prime} \perp}\right|^{2}+m_{\pi}^{2}}{P_{\pi^{\prime}}^{+}}-\frac{\left|\mathbf{P}_{\pi \perp}\right|^{2}+m_{\pi}^{2}}{P_{\pi}^{+}} .
$$

Hence, the constraint $q^{-}<0$ is obviously fulfilled for 
any value of $\mathbf{P}_{\pi \perp}$, since $\left|\mathbf{P}_{\pi^{\prime} \perp}\right|=\left|\mathbf{P}_{\pi \perp}\right|$ and $P_{\pi^{\prime}}^{+}=q^{+}+$ $P_{\pi}^{+}>P_{\pi}^{+}$. From Eq. (73) one has

$$
q^{2}=-\left(q^{+}\right)^{2} \frac{\left|\mathbf{P}_{\pi \perp}\right|^{2}+m_{\pi}^{2}}{P_{\pi}^{+}\left(q^{+}+P_{\pi}^{+}\right)}=-\frac{\left|\mathbf{P}_{\pi \perp}\right|^{2}+m_{\pi}^{2}}{x_{\pi}\left(1+x_{\pi}\right)},
$$

where $x_{\pi}=P_{\pi}^{+} / q^{+}$. Therefore, once a value for $\left|\mathbf{P}_{\pi \perp}\right|$ is chosen, $P_{\pi}^{+}$and $P_{\pi^{\prime}}^{+}$are fixed. For a purely longitudinal motion of the pions, i.e. $\mathbf{P}_{\pi \perp}=\mathbf{P}_{\pi^{\prime} \perp}=\mathbf{0}$, it is easy to obtain from Eq. (74) that

$$
\begin{aligned}
& P_{\pi}^{+}=q^{+}\left(-\frac{1}{2}+\sqrt{\frac{1}{4}-\frac{m_{\pi}^{2}}{q^{2}}}\right) \text { and } \\
& P_{\pi^{\prime}}^{+}=q^{+}\left(\frac{1}{2}+\sqrt{\left.\frac{1}{4}-\frac{m_{\pi}^{2}}{q^{2}}\right) .}\right.
\end{aligned}
$$

In the limit of $m_{\pi}=0$, the longitudinal momenta of the pions according to Eq. (75) are

$$
P_{\pi}^{+}=0 \quad \text { and } \quad P_{\pi^{\prime}}^{+}=q^{+}
$$

for any value of the momentum transfer.

In a frame where $q^{+} \neq 0$, the electromagnetic current $j^{+}$in the spacelike region, Eq. (20), receives contributions from the valence component of the wave function, $j^{(\mathrm{I})+}$ given by Eq. (56), as well as from the nonvalence components, $j^{(\mathrm{II})+}$ of Eq. (64), i.e. from the $Z$-diagram contribution (see Fig. 7).
The contribution of the pion valence wave function to the current can be calculated from Eq. (56) introducing the plus component of the operator $\Gamma^{\mu}\left(k-P_{\pi}, q\right)$ for $k^{-}=$ $k_{\mathrm{on}}^{-}$, as given in Eq. (41) and discussed in Sec. V B, once the values of the pion vertex functions $\left[\bar{\Lambda}_{\pi^{\prime}}\left(k^{\prime}, P_{\pi^{\prime}}\right)\right]_{k^{\prime-}=k_{\mathrm{on}}^{\prime-}}$ and $\left[\Lambda_{\pi}\left(P_{\pi}-k^{\prime}, P_{\pi}\right)\right]_{k^{\prime-}=k_{\mathrm{on}}^{\prime-}}$ in the instantaneous terms have been specified.

In the limit of zero pion mass, according to Eq. (56) the valence contribution to the spacelike pion form factor vanishes, since $P_{\pi}^{+}=0$. Then, only the $Z$-diagram contribution survives in this limit, as in the timelike region.

The contribution of the $Z$ diagram to the elastic pion form factor can be obtained from Eq. (72) by substituting in Eq. (64) the pion absorption vertex of Eq. (43). The result can be written as follows:

$$
F^{\mathrm{II}}\left(q^{2}\right)=\sum_{n} \frac{f_{V n}}{q^{2}-M_{n}^{2}} f_{n}^{\mathrm{II}}\left(q^{2}\right) .
$$

Since $f_{n}^{\mathrm{II}}\left(q^{2}\right)$ is invariant under kinematical LF boosts, we choose to evaluate the contribution of each vector meson, $f_{n}^{\mathrm{II}}\left(q^{2}\right)$, in the same reference frame that we used in the timelike region, i.e., we adopt the rest frame for each resonance $\left(q^{+}=M_{n}, \mathbf{q}_{\perp}=0 ; P_{n}^{+}=q^{+}=M_{n}, P_{n}^{-}=\right.$ $\left.M_{n}^{2} / q^{+}=M_{n}\right)$.

Then for a finite value of the pion mass and taking advantage of Eq. (G3), we have

$$
\begin{aligned}
f_{n}^{\mathrm{II}}\left(q^{2}\right)= & \sqrt{2} \frac{N_{c}}{8 \pi^{3}} \frac{\epsilon_{z}^{+}}{P_{\pi^{\prime}}^{+}+P_{\pi}^{+}} \int_{0}^{q^{+}} \frac{d k^{+}}{k^{+}\left(q^{+}-k^{+}\right)\left(P_{\pi}^{+}+k^{+}\right)} \int d \mathbf{k}_{\perp} \mathcal{D}_{\pi}\left\{\frac{\psi_{n}\left(k^{+}, \mathbf{k}_{\perp} ; P_{n}^{+}, \mathbf{0}_{\perp}\right)\left[M_{n}^{2}-M_{0}^{2}\left(k^{+}, \mathbf{k}_{\perp} ; P_{n}^{+}, \mathbf{0}_{\perp}\right)\right]}{\left[q^{2}-M_{0}^{2}\left(k^{+}, \mathbf{k}_{\perp} ; q^{+}, \mathbf{0}_{\perp}\right)+i \epsilon\right]}\right. \\
& \left.\times\left[\mathcal{T}_{\text {on, }(2, n)}^{\prime}+\mathcal{T}_{1,(2, n)}^{\prime}\right]+\left[\Lambda_{n}\left(k, P_{n}\right)\right]_{k^{-}=q^{-}+(k-q)_{\text {on }}^{-}} \mathcal{T}_{3,(2, n)}^{\prime}\right\}
\end{aligned}
$$

with

$$
\begin{gathered}
\mathcal{T}_{\text {on },(2, n)}^{\prime}=\psi_{\pi^{\prime}}^{*}\left(\left(k^{+}+P_{\pi}^{+}\right),\left(\mathbf{k}+\mathbf{P}_{\pi}\right)_{\perp} ; P_{\pi^{\prime}}^{+}, \mathbf{P}_{\pi^{\prime} \perp}\right) \operatorname{Tr}\left[\left[\left(k+\not P_{\pi}\right)_{\mathrm{on}}+m\right] \gamma^{5}\left[(k-\not 1)_{\mathrm{on}}+m\right]\left[\hat{V}_{n z}\left(k, k-P_{n}\right)\right]_{\mathrm{on}}\left(k_{\mathrm{on}}+m\right) \gamma^{5}\right], \\
\mathcal{T}_{1,(2, n)}^{\prime}=\frac{1}{2} \frac{m}{f_{\pi}}\left[\bar{\Lambda}_{\pi^{\prime}}\left(k+P_{\pi}, P_{\pi^{\prime}}\right)\right]_{k^{-}=q^{-}+(k-q)_{\mathrm{on}}^{-}} \operatorname{Tr}\left[\gamma^{+} \gamma^{5}\left[(k-\not)_{\mathrm{on}}+m\right]\left[\hat{V}_{n z}\left(k, k-P_{n}\right)\right]_{\mathrm{on}}\left(k_{\mathrm{on}}+m\right) \gamma^{5}\right], \\
\mathcal{T}_{3,(2, n)}^{\prime}=\frac{1}{2} \psi_{\pi \prime}^{*}\left(\left(k^{+}+P_{\pi}^{+}\right),\left(\mathbf{k}+\mathbf{P}_{\pi}\right)_{\perp} ; P_{\pi^{\prime}}^{+}, \mathbf{P}_{\pi^{\prime} \perp}\right) \operatorname{Tr}\left[\left[\left(k+\not P_{\pi}\right)_{\mathrm{on}}+m\right] \gamma^{5}\left[(k-\not)_{\mathrm{on}}+m\right]\left[\hat{V}_{n z}\left(k, k-P_{n}\right)\right]_{\mathrm{on}} \gamma^{+} \gamma^{5}\right] .
\end{gathered}
$$

The Dirac structure $\left[\hat{V}_{n z}\left(k, k-P_{n}\right)\right]_{\text {on }}$ for the ${ }^{3} S_{1}$ meson state is given by Eq. (G13). As noted in Appendix G, in our reference frame one has $\epsilon_{z}^{+}=1$. Eqs. (80) and (81) represent the instantaneous contributions. Analogously to the timelike case, for a vanishing pion mass one has $\widetilde{\mathcal{T}}_{\text {on, }(2, n)}^{\prime}=0$ [see Eq. (G18)].

Let us now evaluate $f_{n}^{\mathrm{II}}\left(q^{2}\right)$ at $q^{2} \rightarrow 0^{-}$for a finite value of the mass of the pion. To begin with, we consider: (i) a constant value for $\mathcal{D}_{\pi}$, (ii) a simple form for the LF pion wave function [34]

$$
\begin{aligned}
& \psi_{\pi^{\prime}}\left[\left(k^{+}+P_{\pi}^{+}\right),\left(\mathbf{k}_{\perp}+\mathbf{P}_{\pi \perp}\right) ; P_{\pi^{\prime}}^{+}, \mathbf{P}_{\pi^{\prime} \perp}\right] \\
& \quad=\frac{m}{f_{\pi}} \frac{P_{\pi^{\prime}}^{+}}{m_{\pi}^{2}-M_{0 \pi^{\prime}}^{2}\left(k^{+}+P_{\pi}^{+}, \mathbf{k}_{\perp}+\mathbf{P}_{\pi \perp} ; P_{\pi^{\prime}}^{+}, \mathbf{P}_{\pi^{\prime} \perp}\right)},
\end{aligned}
$$


and (iii) in the instantaneous term (80) take $\left[\bar{\Lambda}_{\pi^{\prime}}(k+\right.$ $\left.\left.P_{\pi}, P_{\pi^{\prime}}\right)\right]_{k^{-}=q^{-}+(k-q)_{\text {on }}^{-}}$proportional to $\psi_{\pi^{\prime}}^{*}\left[\left(k^{+}+\right.\right.$ $\left.\left.P_{\pi}^{+}\right),\left(\mathbf{k}_{\perp}+\mathbf{P}_{\pi \perp}\right) ; P_{\pi^{\prime}}^{+}, \mathbf{P}_{\pi^{\prime} \perp}\right]$ (see the next section). For a finite value of the mass of the pion, let us note that in the limit $q^{2} \rightarrow 0^{-}$from Eq. (75) one obtains $P_{\pi}^{+} \rightarrow \infty$ and $P_{\pi^{\prime}}^{+}=\left(M_{n}+P_{\pi}^{+}\right) \rightarrow \infty$. Then, since the squared free mass for the final pion is

$$
\begin{aligned}
& M_{0 \pi^{\prime}}^{2}\left[\left(k^{+}+P_{\pi}^{+}\right),\left(\mathbf{k}_{\perp}+\mathbf{P}_{\pi \perp}\right) ; P_{\pi^{\prime}}^{+}, \mathbf{P}_{\pi^{\prime} \perp}\right] \\
& \quad=P_{\pi^{\prime}}^{+}\left(\frac{\left|\mathbf{k}_{\perp}\right|^{2}+m^{2}}{P_{\pi}^{+}+k^{+}}+\frac{\left|\mathbf{k}_{\perp}\right|^{2}+m^{2}}{P_{\pi^{\prime}}^{+}-P_{\pi}^{+}-k^{+}}\right),
\end{aligned}
$$

it becomes large for $P_{\pi}^{+} \rightarrow \infty$, i.e. $M_{0 \pi^{\prime}}^{2} \sim P_{\pi^{\prime}}^{+}$. Then $\psi_{\pi^{\prime}}^{*}\left[\left(k^{+}+P_{\pi}^{+}\right),\left(\mathbf{k}_{\perp}+\mathbf{P}_{\pi \perp}\right) ; P_{\pi^{\prime}}^{+}, \mathbf{P}_{\pi^{\prime} \perp}\right]$ becomes a constant for $P_{\pi^{\prime}}^{+} \rightarrow \infty$. Furthermore, for $P_{\pi}^{+} \rightarrow \infty$ the traces in Eqs. (79) and (80) are proportional to $\sim P_{\pi}^{+}$. Therefore, collecting together the factors $P_{\pi}^{+}$in Eq. (78), one concludes that for a finite value of the pion mass $\lim _{q^{2} \rightarrow 0^{-}} f_{n}^{\mathrm{II}}\left(q^{2}\right) \sim \lim _{q^{2} \rightarrow 0^{-}} 1 / P_{\pi}^{+}=0$. The same result, $\lim _{q^{2} \rightarrow 0^{-}} f_{n}^{\mathrm{II}}\left(q^{2}\right)=0$, should also hold for pion wave functions which are eigenfunctions of a Hamiltonian [35].

On the contrary, in the limit of $m_{\pi}=0$, the longitudinal momenta of the pions are $P_{\pi}^{+}=0$ and $P_{\pi^{\prime}}^{+}=M_{n}$, respec- tively [see Eq. (75)]. Then, according to Eq. (56), the valence contribution to the spacelike pion form factor vanishes, while the $Z$ diagram yields a nonzero contribution.

A comment is appropriate here. In the work of Ref. [21], where $m_{\pi} \neq 0$, it was found that the wave-function contribution to the spacelike pion form factor strongly decreases in the frame $q^{+}=\sqrt{-q^{2}}$ as the momentum transfer $-q^{2}$ increases. As a consequence, the $Z$-diagram contribution, which is zero at $q^{2}=0$, becomes the dominant one at high momentum transfer. As the pion mass is artificially decreased in that model, we find that the momentum at which the $Z$ diagram starts to dominate the form factor tends toward zero, in agreement with the previous discussion.

Since in this paper we work at the limit of a vanishing pion mass, in our reference frame, the full spacelike pion form factor is given by $F^{\mathrm{II}}\left(q^{2}\right)$ alone. It has to be noted that, as occurs in the timelike region and for the same reasons (see Appendix G), for $m_{\pi}=0$ only the instantaneous terms $\mathcal{T}_{1,(2, n)}^{\prime}$ and $\mathcal{T}_{3,(2, n)}^{\prime}$ [cf. Eqs. (80) and (81)] give contribution to the pion form factor. These terms can be written in the following form:

$$
\begin{gathered}
\mathcal{T}_{1,(2, n)}^{\prime}=-\frac{1}{2} \frac{m}{f_{\pi}}\left[\bar{\Lambda}_{\pi^{\prime}}\left(k+P_{\pi}, P_{\pi^{\prime}}\right)\right]_{k^{-}=q^{-}+(k-q)_{\text {on }}^{-}} \operatorname{Tr}\left[\gamma^{+}\left[(k-\not)_{\text {on }}+m\right]\left[\hat{V}_{n z}\left(k, k-P_{n}\right)\right]_{\text {on }}\left(k_{\text {on }}+m\right)\right], \\
\mathcal{T}_{3,(2, n)}^{\prime}=\frac{1}{2} \psi_{\pi^{\prime}}^{*}\left(k^{+}, \mathbf{k}_{\perp} ; M_{n}, \mathbf{0}_{\perp}\right) \operatorname{Tr}\left[\left[-k_{\text {on }}+m\right]\left[(k-\not)_{\text {on }}+m\right]\left[\hat{V}_{n z}\left(k, k-P_{n}\right)\right]_{\text {on }} \gamma^{+}\right] .
\end{gathered}
$$

\section{A LIGHT-FRONT MODEL}

To evaluate the pion form factor we need

(i) A model for the HLFD pion and vector-meson wave functions which appear in Eqs. (70) and (78);

(ii) A value for the probability, $P_{q \bar{q}, n}$, of the VM valence component (see Appendixes $\mathrm{E}$ and $\mathrm{F}$ );

(iii) An approximation for the pion vertex functions which represent the pion emission or absorption by a quark;

(iv) To assign a value to the instantaneous pion and VM vertex functions.

\section{A. Model wave functions and probability of valence components}

The vector-meson resonances are described by an effective light-front model inspired by QCD [35], that can be also applied to the pion. The model retains the main features of the meson spectra and allows one to perform simple numerical calculations. The squared mass operator for the $S$ mesons contains a harmonic oscillator interaction featuring the confinement and a Dirac delta function that acts in the ${ }^{1} S_{0}$ channel (with a renormalized strength). The wave functions for the ${ }^{3} S_{1}$ channel are solutions of the following eigenvalue problem

$$
\left[4\left(|\kappa|^{2}+m^{2}\right)+\frac{1}{64} \omega^{2} r^{2}+a\right] \Psi_{n}^{\mathrm{HO}}(\mathbf{r})=M_{n}^{2} \Psi_{n}^{\mathrm{HO}}(\mathbf{r}),
$$

where $|\kappa|^{2}=M_{0}^{2} / 4-m^{2}$ is the square of the intrinsic quark three-momentum, $M_{n}^{2}=n \omega+M_{\rho}^{2}$ and the eigenfunctions $\Psi_{n}^{\mathrm{HO}}(\mathbf{r})$ are the three-dimensional harmonic oscillator wave functions for zero angular momentum. The HLFD wave functions, without the Melosh rotations, are given in the Fourier space by

$$
\psi_{n}\left(k^{+}, \mathbf{k}_{\perp}, P_{n}^{+}, \mathbf{P}_{n \perp}\right)=P_{n}^{+} \Psi_{n}^{\mathrm{HO}}\left(|\kappa|^{2}\right) .
$$

The factor $P_{n}^{+}$comes from the different normalizations used for $\psi_{n}\left(k^{+}, \mathbf{k}_{\perp}, P_{n}^{+}, \mathbf{P}_{n \perp}\right)$ and $\Psi_{n}^{\mathrm{HO}}\left(|\kappa|^{2}\right)$. Indeed the function $\Psi_{n}^{\mathrm{HO}}\left(|\kappa|^{2}\right)$ is normalized through the equation

$$
\int\left|\Psi_{n}^{\mathrm{HO}}\left(|\kappa|^{2}\right)\right|^{2} d^{3} \kappa=1,
$$

while the function $\psi_{n}\left(k^{+}, \mathbf{k}_{\perp}, P_{n}^{+}, \mathbf{P}_{n \perp}\right)$ is normalized through the evaluation of the charge form factor of a vector meson at $q^{2}=0$, i.e. by using the so-called charge nor- 
malization (Appendix E), more appropriate in a relativistic context [30]. In the actual calculation, we have to consider that, after properly integrating the valence component, its probability should be recovered. This amounts to construct a schematic model for the probability $P_{n, q \bar{q}}$ for each excited state (see Appendix F), and subsequently to renormalize $\psi_{n}\left(k^{+}, \mathbf{k}_{\perp}, P_{n}^{+}, \mathbf{P}_{n \perp}\right)$ in Eq. (87) as follows:

$$
\psi_{n}\left(k^{+}, \mathbf{k}_{\perp}, P_{n}^{+}, \mathbf{P}_{n \perp}\right)=\sqrt{P_{n, q \bar{q}}} P_{n}^{+} \Psi_{n}^{\mathrm{HO}}\left(|\kappa|^{2}\right) .
$$

In the model of Ref. [35] the complete form of the pion wave function is an eigenstate of the mass operator of Eq. (86) plus a Dirac-delta interaction (in the configuration space), which is necessary for producing a pion with a small mass (i.e. a collapsing $q \bar{q}$ pair in the ${ }^{1} S_{0}$ channel). The pion wave function is found from the pole of the resolvent, explicitly written in Ref. [35]. The result is the following:

$$
\psi_{\pi}\left(k^{+}, \mathbf{k}_{\perp}, P_{\pi}^{+}, \mathbf{P}_{\pi \perp}\right)=P_{\pi}^{+} \sum_{n} \frac{\Psi_{n}^{\mathrm{HO}}\left(|\kappa|^{2}\right) \Psi_{n}^{\mathrm{HO}}(0)}{m_{\pi}^{2}-M_{n}^{2}},
$$

where $\Psi_{n}^{\mathrm{HO}}(0)$ is the $S$-wave harmonic oscillator eigenfunction in coordinate space at the origin.

In this model, the pion wave function approaches the asymptotic limit, Eq. (82), imposed by the presence of the Dirac-delta function in the interaction.

The relativistic constituent quark model of Ref. [35] achieves a satisfactory description of the experimental masses for both singlet and triplet $S$-wave mesons, with a natural explanation of the "Iachello-Anisovitch law" [36,37], namely, the almost linear relation between the square mass of the excited states and the radial quantum number $n$. Since the model does not include the mixing between isoscalar and isovector mesons, in this paper we include only the contributions of the isovector $\rho$-like vector mesons.

Our model calculation can be repeated by using a different set of meson wave functions like, e.g., the one of Ref. [52], to study the dependence of the results from the choice of the meson Hamiltonian.

\section{B. Vertex functions for pion emission or absorption}

As already discussed in Sec. VI, we approximate the pion vertex functions which represent the antipion and pion emission by a quark, as well as the quark-pion absorption vertex by means of a constant

$$
\overline{\mathcal{D}}_{\pi}=\mathcal{D}_{\bar{\pi}}=\frac{m}{f_{\pi}} \lambda_{\pi} ; \quad \text { and } \quad \mathcal{D}_{\pi}=\frac{m}{f_{\pi}} \lambda_{\pi}
$$

in agreement with the constant form adopted in Refs. $[17,18]$ and successfully tested in the study of the pseudoscalar meson decays [18]. The actual value of the constant $\lambda_{\pi}$ is fixed by the pion charge normalization.

It is worth noting that such an approximation appears, $a$ posteriori, to be a reliable one from the direct comparison of our calculations and the experimental data (see Sec. X). Moreover, its reliability could be inferred from the observation that the range of integration in the actual calculation of the form factors is constrained at small values of $\kappa$ by the phase space and at high values of $\kappa$ by the Gaussian falloff of the meson wave functions.

\section{Instantaneous vertex functions}

As anticipated in Secs. VII and VIII, to simplify our calculations we are going to use $m_{\pi}=0$. Within this assumption, for the timelike form factor only the instantaneous contributions $\mathcal{T}_{1,(2, n)}$ and $\mathcal{T}_{3,(2, n)}$ survive, while for the spacelike form factor only the instantaneous terms $\mathcal{T}_{1,(2, n)}^{\prime}$ and $\mathcal{T}_{3,(2, n)}^{\prime}$ remain. Then to fully evaluate the pion form factor in the timelike and in the spacelike region we have still to assign a value to the instantaneous pion and VM vertex functions, i.e. to the vertex functions $\left[\Lambda_{\bar{\pi}}(k-\right.$ $\left.\left.P_{\pi}, P_{\bar{\pi}}\right)\right]_{k^{-}=q^{-}+(k-q)_{\text {on }}^{-}}$and $\left[\bar{\Lambda}_{\pi^{\prime}}\left(k+P_{\pi}, P_{\pi^{\prime}}\right)\right]_{k^{-}=q^{-}+(k-q)_{\text {on }}^{-}}$ in Eqs. (G19) and (84), respectively, and to the vertex function $\left[\Lambda_{n}\left(k, P_{n}\right)\right]_{k^{-}=q^{-}+(k-q)_{\text {on }}^{-}}$of Eqs. (G20) and (85). The instantaneous contributions to the timelike pion form factor corresponding to the vertex functions $\left[\Lambda_{\bar{\pi}}(k-\right.$ $\left.\left.P_{\pi}, P_{\bar{\pi}}\right)\right]_{k^{-}=q^{-}+(k-q)_{\text {on }}^{-}}$and $\left[\Lambda_{n}\left(k, P_{n}\right)\right]_{k^{-}=q^{-}+(k-q)_{\text {on }}^{-}}$are represented by diagrams (a) and (b) of Fig. 8, respectively.

Let us note that the presence of the factors $\left(k^{+} \pm P_{\pi}^{+}\right)$ and $k^{+}$in the denominators of the two instantaneous terms produces an enhancement of the contributions around the values $\left(k^{+} \pm P_{\pi}^{+}\right)=0$ and $k^{+}=0$ in the $k^{+}$integration. Within our assumption of a vanishing pion mass, this means that, for both the instantaneous terms, there is an enhancement of the contribution at the end point $k^{+}=0$, which corresponds to an infinite value of the $z$ component of the intrinsic quark three-momentum, $\kappa_{z}=M_{0}(2 x-$ 1) $/ 2\left(\kappa_{z}=-\infty\right.$ for $x=0$, since $\left.M_{0} \rightarrow \infty\right)$. Therefore the high momentum part of the meson vertex functions, i.e. the short-range part in coordinate space, is very relevant. Then in the instantaneous vertex functions $\Lambda_{\pi(n)}^{\text {ist }}$ we
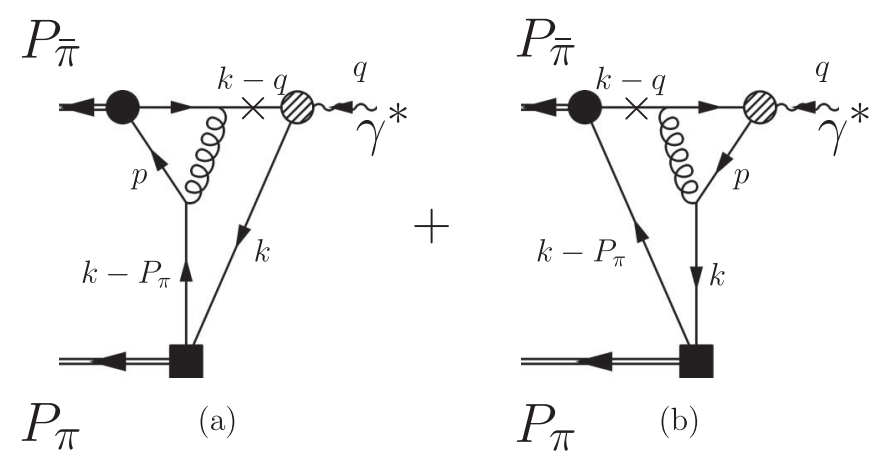

FIG. 8. Instantaneous contributions to the timelike em form factor of a massless pion. The instantaneous interaction is attached to the pion vertex in (a) and to VM vertex in (b). The dashed circle represents the dressed photon vertex (see Fig. 4 for details) 
assume that the very short-range part of the one-gluonexchange interaction, which includes spin-spin terms [53], is the dominant one. In symbolic notation we have (see Fig. 8):

$$
\Lambda^{\text {ist }}=\mathcal{K}^{\text {ist }} G_{0} \Lambda^{\text {full }},
$$

where $\mathcal{K}^{\text {ist }}$ is the Bethe-Salpeter kernel for the instantaneous vertex function $\Lambda^{\text {ist }}, G_{0}$ the propagator of two free quarks, and $\Lambda^{\text {full }}$ the full vertex function.

The kernel $\mathcal{K}^{\text {ist }}$ is assumed to be dominated by the short-range part of the interaction. Actually we drastically simplify Eq. (92) as follows:

$$
\Lambda^{\text {ist }} \sim c \Lambda^{\text {full }} .
$$

This amounts to naively assume that $\Lambda^{\text {full }}$ is an eigenstate of $\mathcal{K}^{\text {ist }} G_{0}$. Furthermore, we assume that $\Lambda^{\text {full }}$ is still related to the LF meson wave function as illustrated in Sec. IV, i.e. $\Lambda_{\pi(n)}^{\text {full }}=\psi_{\pi(n)}\left[M_{\pi(n)}^{2}-M_{0}^{2}\right] / P_{\pi(n)}^{+}$.

The constant $c$ is thought to roughly describe the effects of the short-range interaction. In particular, if we take grossly into account only the spin-spin interaction term, then the results of Ref. [26] are recovered (i) by choosing $c=-3 / 4$ for the pion vertex function [Fig. 8(a)] and $c=$ $1 / 4$ for the VM vertex function [Fig. 8(b)] and (ii) by using the probabilities $P_{q \bar{q} ; n}=\delta \omega^{1 / 2} / 2 \sqrt{2 n+(3 / 2)}$ for the VM valence components with $\delta \omega^{1 / 2} / 2=1$ (see Appendix F). With this choice for the constants $c$ 's, the relative weight of the VM instantaneous terms with respect to the pion instantaneous terms is equal to $-1 / 3$. At variance with Ref. [26], in the present paper we use this relative weight, $w_{\mathrm{VM}}=c_{\mathrm{VM}} / c_{\pi}$, as a free parameter.

In conclusion, we replace the momentum component of the pion vertex function in Eq. (G19) as follows:

$\frac{m}{f_{\pi}}\left[\Lambda_{\bar{\pi}}\left(k-P_{\pi}, P_{\bar{\pi}}\right)\right]_{k^{-}=q^{-}+(k-q)_{\text {on }}^{-}} \rightarrow \frac{c_{\pi}}{P_{\bar{\pi}}^{+}} \psi_{\bar{\pi}}\left(k^{+}-P_{\pi}^{+}, \mathbf{k}_{\perp}-\mathbf{P}_{\pi \perp} ; P_{\bar{\pi}}^{+}, \mathbf{P}_{\bar{\pi} \perp}\right)\left[m_{\pi}^{2}-M_{0}^{2}\left(k^{+}-P_{\pi}^{+}, \mathbf{k}_{\perp}-\mathbf{P}_{\pi \perp} ; P_{\bar{\pi}}^{+}, \mathbf{P}_{\bar{\pi} \perp}\right)\right]$,

and in Eq. (84) as follows:

$$
\begin{aligned}
\frac{m}{f_{\pi}}\left[\bar{\Lambda}_{\pi^{\prime}}\left(k+P_{\pi}, P_{\pi^{\prime}}\right)\right]_{k^{-}=q^{-}+(k-q)_{\text {on }}^{-}} \rightarrow & \frac{c_{\pi}}{P_{\pi^{\prime}}^{+}} \psi_{\pi^{\prime}}^{*}\left(k^{+}+P_{\pi}^{+}, \mathbf{k}_{\perp}+\mathbf{P}_{\pi \perp} ; P_{\pi^{\prime}}^{+}, \mathbf{P}_{\pi^{\prime} \perp}\right) \\
& \times\left[m_{\pi}^{2}-M_{0}^{2}\left(k^{+}+P_{\pi}^{+}, \mathbf{k}_{\perp}+\mathbf{P}_{\pi \perp} ; P_{\pi^{\prime}}^{+}, \mathbf{P}_{\pi^{\prime} \perp}\right)\right] .
\end{aligned}
$$

The momentum component of the VM vertex function in Eqs. (G20) and (85) is approximated by

$$
\left[\Lambda_{n}\left(k, P_{n}\right)\right]_{k^{-}=q^{-}+(k-q)_{\mathrm{on}}^{-}} \rightarrow \frac{c_{\mathrm{VM}}}{P_{n}^{+}} \psi_{n}\left(k^{+}, \mathbf{k}_{\perp} ; P_{n}^{+}, \mathbf{P}_{n \perp}\right)\left[M_{n}^{2}-M_{0}^{2}\left(k^{+}, \mathbf{k}_{\perp} ; P_{n}^{+}, \mathbf{P}_{n \perp}\right)\right] .
$$

As explained in the previous sections, in the limit of a vanishing pion mass both in the timelike and in the spacelike case one has $P_{\pi}^{+}=0$ and $P_{\pi^{\prime}}^{+}=P_{\bar{\pi}}^{+}=M_{n}$. Then, the quantities $g_{V n}^{+}\left(q^{2}\right)$ of Eq. (70) and $f_{n}^{\mathrm{II}}\left(q^{2}\right)$ of Eq. (78) acquire the same functional form, despite the sign of $q^{2}$, and reduce to the same function $\xi_{n}\left(q^{2}\right)$ :

$$
\begin{aligned}
\xi_{n}\left(q^{2}\right)= & \frac{N_{c}}{16 \pi^{3}} \frac{m}{f_{\pi}} \lambda_{\pi} c_{\pi} \frac{\sqrt{2}}{M_{n}^{2}} \int_{0}^{M_{n}} \frac{d k^{+}}{\left(k^{+}\right)^{2}\left(M_{n}-k^{+}\right)} \int d \mathbf{k}_{\perp}\left[\mathcal{T}_{1, n}\left(k^{+}, \mathbf{k}_{\perp}\right)+\mathcal{T}_{3, n}\left(k^{+}, \mathbf{k}_{\perp}\right)\right] \psi_{\pi^{\prime}}^{*}\left(k^{+}, \mathbf{k}_{\perp} ; M_{n}, \mathbf{0}_{\perp}\right) \\
& \times\left[M_{n}^{2}-M_{0}^{2}\left(k^{+}, \mathbf{k}_{\perp} ; M_{n}, \mathbf{0}_{\perp}\right)\right] \psi_{n}\left(k^{+}, \mathbf{k}_{\perp}, M_{n}, \mathbf{0}_{\perp}\right),
\end{aligned}
$$

where $\mathcal{T}_{1, n}$ and $\mathcal{T}_{3, n}$ are given by

$$
\begin{gathered}
\mathcal{T}_{1, n}=-\frac{\left[m_{\pi}^{2}-M_{0}^{2}\left(k^{+}, \mathbf{k}_{\perp} ; M_{n}, \mathbf{0}_{\perp}\right)\right]}{\left[q^{2}-M_{0}^{2}\left(k^{+}, \mathbf{k}_{\perp} ; M_{n}, \mathbf{0}_{\perp}\right)+i \epsilon\right]} \operatorname{Tr}\left[\gamma^{+}\left[(k-\not)_{\mathrm{on}}+m\right]\left[\hat{V}_{n z}\left(k, k-P_{n}\right)\right]_{\mathrm{on}}\left(k_{\mathrm{on}}+m\right)\right] \\
=-4 \frac{\left[m_{\pi}^{2}-M_{0}^{2}\left(k^{+}, \mathbf{k}_{\perp} ; M_{n}, \mathbf{0}_{\perp}\right)\right]}{\left[q^{2}-M_{0}^{2}\left(k^{+}, \mathbf{k}_{\perp} ; M_{n}, \mathbf{0}_{\perp}\right)+i \epsilon\right]}\left[k^{+}(k-q)_{\mathrm{on}, z}+(k-q)_{\mathrm{on}} \cdot k_{\mathrm{on}}+\left(k^{+}-M_{n}\right) k_{\mathrm{on}, z}-m^{2}\right. \\
\left.\quad-m\left(2 k^{+}-M_{n}\right)\left(k_{\mathrm{on}}-(q-k)_{\mathrm{on}}\right)_{z} H_{S}\left(M_{0}\right)\right] \\
\mathcal{T}_{3, n}=w_{\mathrm{VM}} \operatorname{Tr}\left[\left[-k_{\mathrm{on}}+m\right]\left[(k-\not)_{\mathrm{on}}+m\right]\left[\hat{V}_{n z}\left(k, k-P_{n}\right)\right]_{\mathrm{on}} \gamma^{+}\right] \\
=w_{\mathrm{VM}} 4\left[-k^{+}(k-q)_{\mathrm{on}, z}+(k-q)_{\mathrm{on}} \cdot k_{\mathrm{on}}+\left(k^{+}-M_{n}\right) k_{\mathrm{on}, z}-m^{2}+m M_{n}\left[k_{\mathrm{on}}-(q-k)_{\mathrm{on}}\right]_{z} H_{S}\left(M_{0}\right)\right] .
\end{gathered}
$$


In the last steps in Eqs. (98) and (99) the traces have been explicitly evaluated and the function $H_{S}\left(M_{0}\right)$ is given by

$$
H_{S}\left(M_{0}\right)=\frac{1}{M_{0}+2 m} .
$$

Actually the value of $c_{\pi}$ together with the value of $\lambda_{\pi}$ is fixed by the charge normalization and we have to assign a value only to the relative weight $w_{\mathrm{VM}}$.

In Eq. (97) there is no divergence from the poles at the end points $k^{+}=0$ and $q^{+}-k^{+}=0$, because of the Gaussian decrease of the used VM wave functions at these end points, which correspond to infinite values of the $z$ component of the intrinsic quark three-momentum, $\kappa_{z}=$ $M_{0}(2 x-1) / 2\left(\kappa_{z}=-\infty\right.$ or $\kappa_{z}=+\infty$ for $x=0$ or $x=1$, respectively).

Finally, both in the timelike and in the spacelike regions, the pion electromagnetic form factor can be written as

$$
F_{\pi}\left(q^{2}\right)=\sum_{n} \frac{f_{V n}}{\left[q^{2}-M_{n}^{2}+\imath M_{n} \tilde{\Gamma}_{n}\left(q^{2}\right)\right]} \xi_{n}\left(q^{2}\right) .
$$

We stress that the pion form factor is continuous at $q^{2}=0$ in the limit $m_{\pi} \rightarrow 0$ and that only the instantaneous terms contribute in this limit. We would like to remind the reader that the vector-meson wave functions are normalized to the probability of the valence component, which can be roughly estimated in a simple model, as shown in Appendix F. The decreasing probability of the valence component for the excited vector-meson states is essential to make convergent the sum over the resonances.

\section{RESULTS}

The pion electromagnetic form factor is calculated through Eqs. (97) and (101), where the pion and vectormeson wave functions are eigenstates of the square mass operator defined in Eq. (86) (shown for the vector channel only).

In our calculation we have a small set of parameters: (i) the constituent quark mass, (ii) the oscillator strength $\omega$, (iii) the widths for the vector mesons, $\Gamma_{n}$, and (iv) the relative weight $w_{\mathrm{VM}}$ of the two instantaneous contributions.

The up-down quark mass is fixed at $0.265 \mathrm{GeV}$ [35] and the oscillator strength is fixed at $\omega=1.556 \mathrm{GeV}^{2}$ of Ref. [35].
For the first four vector mesons, the masses and widths, presented in Table I, are used.

The nontrivial $q^{2}$ dependence of $\xi_{n}\left(q^{2}\right)$ in our microscopical model allows a small shift of the VM masses with respect to the values obtained in the analyses of the experimental data by using Breit-Wigner functions with constant values for $\xi_{n}\left(q^{2}\right)$.

For the radial excitations with $M_{n}>2.150 \mathrm{GeV}$, the mass values corresponding to the model of Ref. [35] are used. For the unknown widths we use a single width as a fitting parameter. We choose the value $\Gamma_{n}=0.15 \mathrm{GeV}$, which presents the best agreement with the compilation of the experimental data of Ref. [56]. We consider 20 resonances in our calculation to obtain stability of the results up to $q^{2}=10(\mathrm{GeV} / c)^{2}$.

The probabilities $P_{q \bar{q}, n}$ of the valence component of the VM states are fixed according to the schematic model of Appendix F [see Eq. (F16) and Table II].

As we discussed in the previous sections, it is also necessary to know the amplitude for the virtual process where a constituent quark radiates or absorbs a pion. This unknown function was first investigated in a phenomenological study of decay processes within LF dynamics [18], where it was approximated by a constant, obtaining a satisfactory description of the experimental data. We followed the approximation proposed in [18] in the calculation of the decay amplitude $\xi_{n}\left(q^{2}\right)$ of Eq. (97). The value of the constant $\lambda_{\pi}$, together with the constant $c_{\pi}$ (see the previous section), is fixed by the charge normalization.

The values of the coupling constants, $f_{V n}$, are calculated using Eq. (A4) of Appendix A from the model VM wave functions. The corresponding partial decay width, $\Gamma_{e^{+} e^{-}}$, for these mesons are calculated from our values of $f_{V n}$ using Eq. (A5) [16] and are reported in Table I. The partial decay widths for the vector mesons are in good agreement with the data, when available [38].

We perform two sets of calculations, to test the effect of the pion wave-function model. In one set we use the asymptotic form of the pion valence wave function, Eq. (82), and in another one we choose the eigenstate of the square mass operator of the model of Ref. [35], given by the pion wave function of Eq. (90).

For a deeper investigation of the model dependence of our results, the calculations could be repeated with different meson wave functions, as the ones of Ref. [52].

TABLE I. Known vector-meson masses, $M_{n}$, and widths, $\Gamma_{n}$, used in the model. The corresponding decay widths into $e^{-} e^{+}$pairs, calculated with the VM valence probabilities $P_{q \bar{q} ; n}$ obtained in Appendix F (see also Table II) and the oscillator strength $\omega=$ $1.556 \mathrm{GeV}^{2}$, are compared with the experimental values from [38]. (See text for details.)

\begin{tabular}{lcccccc}
\hline \hline Meson & $M_{n}(\mathrm{MeV})$ & $M_{n}^{\exp }(\mathrm{MeV})[38]$ & $\Gamma_{n}(\mathrm{MeV})$ & $\Gamma_{n}^{\exp }(\mathrm{MeV})[38]$ & $\Gamma_{e^{+} e^{-}}(\mathrm{KeV})$ & $\Gamma_{e^{+} e^{-}}^{\exp }(\mathrm{KeV})[38]$ \\
\hline$\rho(770)$ & 770 & $775.8 \pm 0.5$ & 146.4 & $146.4 \pm 1.5$ & 6.98 & $7.02 \pm 0.11$ \\
$\rho(1450)$ & $1497[54]$ & $1465.0 \pm 25.0$ & $226[54]$ & $400 \pm 60$ & 1.04 & $1.47 \pm 0.4$ \\
$\rho(1700)$ & 1720 & $1720.0 \pm 20.0$ & 220 & $250 \pm 100$ & 0.98 & $>0.23 \pm 0.1$ \\
$\rho(2150)$ & 2149 & $2149.0 \pm 17$ & $230[55]$ & $363 \pm 50$ & 0.65 & - \\
\hline \hline
\end{tabular}


TABLE II. The vector-meson valence probabilities $P_{q \bar{q} ; n}$ for the first 10 resonances.

\begin{tabular}{lcccccccccc}
\hline \hline $\mathrm{n}$ & 0 & 1 & 2 & 3 & 4 & 5 & 6 & 7 & 8 & 9 \\
\hline$P_{q \bar{q} ; n}$ & 0.77 & 0.31 & 0.29 & 0.27 & 0.22 & 0.18 & 0.18 & 0.18 & 0.17 & 0.16 \\
\hline \hline
\end{tabular}

Furthermore different, more elaborate approximations for the vertices representing pion emission or absorption by a quark or for the instantaneous vertices could be used. We leave these calculations to a future work.

The results for the form factor are shown in Figs. 9-11. In Fig. 9 the results corresponding to the weight $w_{V M}=$ -0.7 are shown, while in Fig. 11 the results corresponding to $w_{V M}=-0.7$ and $w_{V M}=-1.5$ are compared in a linear scale around the $\rho$ meson peak. In Fig. 9 we also report the results calculated with the masses and the widths used in Ref. [26] and reported in Table III. For this calculations the oscillator strength $\omega=1.39 \mathrm{GeV}^{2}$, the probabilities $P_{q \bar{q} ; n}=1 / \sqrt{2 n+(3 / 2)}$ and $\quad c_{\pi}=-3 / 4, \quad c_{V M}=1 / 4$ have been used.

Let us note that our results are the same within a few percent, if in Dirac structure of the $n$th VM vertex [Eq. (16)], the free mass is replaced with $M_{n}$.

In Fig. 9, we show our results in a wide region of square momentum transfers, from -10 up to $10(\mathrm{GeV} / c)^{2}$, comparing them with the data collected by Baldini et al. [56] and with the data of Ref. [57]. A general qualitative agreement with the data is seen in this wide range of momentum transfers, independently of the detailed form of the pion wave function. It has to be stressed that the heights of the
TL bumps directly depend on the calculated values of $f_{V n}$ and $\xi_{n}\left(q^{2}\right)$.

The results obtained with the asymptotic pion wave function and the full model present some difference only above $3(\mathrm{GeV} / c)^{2}$.

The pion form factor is particularly very well described in the spacelike region, both using the weight $w_{V M}=-0.7$ or the weight $w_{V M}=-1.5$, as can be clearly seen in Fig. 10, where the ratio of the SL form factor to the monopole factor $M\left(q^{2}\right)=1 /\left(1-q^{2} / M_{\rho}^{2}\right)$ is shown. The excellent agreement with the experimental form factor at low momentum transfers is expected, since we have built in the generalized $\rho$-meson dominance.

The timelike region between 0 and $3(\mathrm{GeV} / c)^{2}$, where $\rho(770), \rho(1450)$ and $\rho(1700)$ appear, is shown in Fig. 11 in a linear scale. The $\rho$-meson peak is placed at the right position using a bare mass of $770 \mathrm{MeV}$. From this figure it is clear that the parameter $w_{V M}$ is able to control the region of the $\rho(770)$ peak, while in other regions its effect is less relevant. For $w_{V M}=-1.5$, the $\rho(770)$ peak is very well described, except for the region around $2(\mathrm{GeV} / c)^{2}$, where our results underestimate the experimental data. This dip is due to a destructive interference between the contributions of $\rho(770), \rho(1450)$, and $\rho(1700)$, and could be potentially sensitive for a detailed test of the model presently adopted for the meson wave functions and other approximations introduced.

It is clear that the introduction of $\omega$-like and $\phi$-like mesons could improve the description of the data in the TL region. However, a consistent dynamical description of the mixing of isospin states is far beyond the present

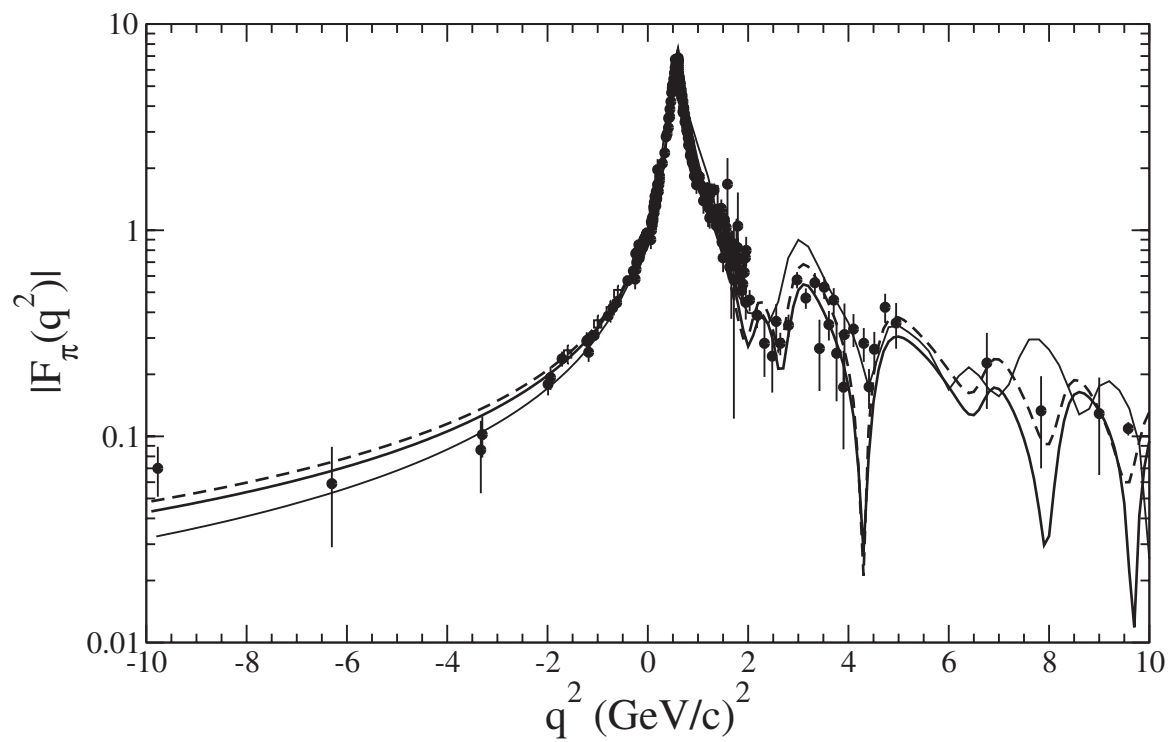

FIG. 9. Pion electromagnetic form factor as a function of the momentum squared $q^{2}$. Results for the asymptotic and the full pion wave functions, obtained with $w_{V M}=-0.7$ (see Sec. IX) and the quantities shown in Table I, are indicated by dashed and solid curves, respectively. The thin solid line represents the result with $w_{V M}=-1 / 3$ and the parameters of Table III. Experimental data are from Ref. [56] (full dots) and Ref. [57] (open squares). 


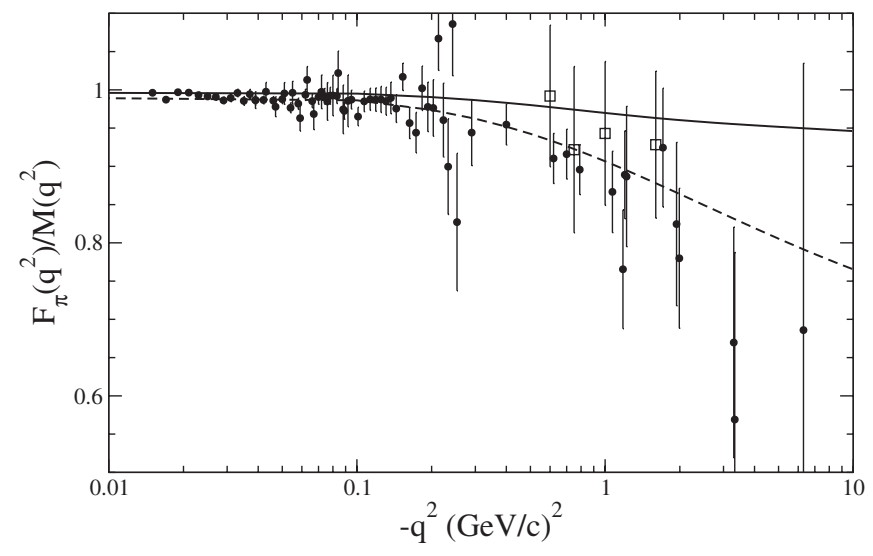

FIG. 10. Spacelike pion electromagnetic form factor divided by the monopole $M\left(q^{2}\right)=1 /\left(1-q^{2} / M_{\rho}^{2}\right)$ vs the momentum squared $q^{2}$. The solid curve corresponds to $w_{V M}=-1.5$ and the dashed line to $w_{V M}=-0.7$, all the other quantities are according to Table I. The experimental data are as in Fig. 9.

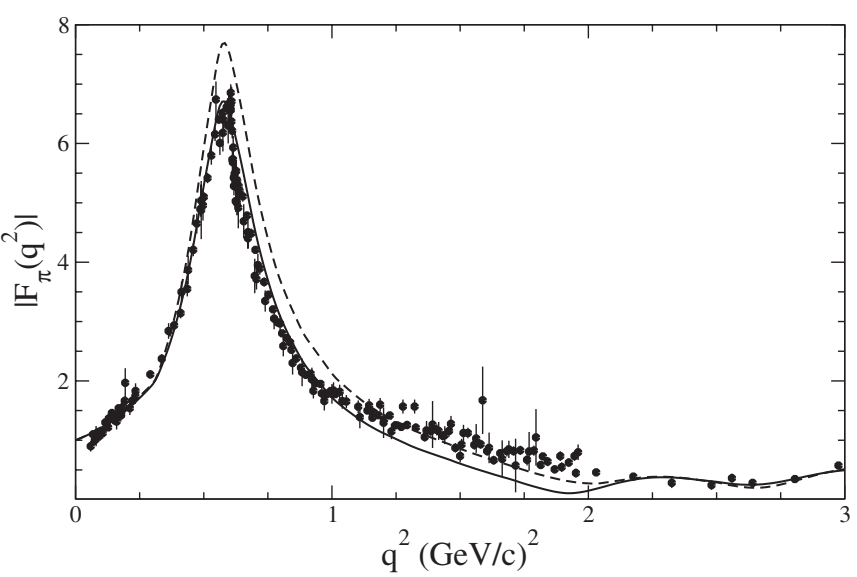

FIG. 11. Pion electromagnetic form factor as a function of the momentum squared $q^{2}$. The solid curve corresponds to $w_{V M}=$ -1.5 and the dashed line to $w_{V M}=-0.7$, all the other quantities are according to Table I. The experimental data are as in Fig. 9.

work, and we leave it for future developments of the model [40].

Finally, we have also calculated the adimensional quantity, $F_{\pi}\left(q^{2}\right)\left|q^{2}\right| / q_{0}^{2}\left(q_{0}=0.77 \mathrm{GeV} / c\right)$, up to $q^{2}=$
$-1000(\mathrm{GeV} / c)^{2}$, observing a smooth decreases from a value of 0.691 for $q^{2}=-100(\mathrm{GeV} / c)^{2}$ to a value of 0.677 for $q^{2}=-1000(\mathrm{GeV} / c)^{2}$.

\section{SUMMARY AND CONCLUSIONS}

In this work, we are able to give a unified description of the pion electromagnetic form factor in the space- and timelike regions, thanks to the choice of a reference frame where $q^{+}>0$. This unified description of the SL and TL form factor is the first one which takes into account the fermionic nature of the constituents quarks and a microscopic description of the hadronic component of the photon wave function.

It should be pointed out that by studying in a common framework the pion SL and TL form factors, we also access information from the radially excited vector-meson wave functions. Indeed, in our approach, in the timelike region the virtual photon couples directly to the vectormeson resonances, which in turn decay in $\pi^{+} \pi^{-}$. Therefore our microscopical model could represent a useful tool to address the investigation of the vector-meson Green function.

The main steps are shortly summarized. As a method to derive the pion form factor within the LF dynamics in an arbitrary reference frame, we integrate over the light-front energy, $k^{-}$, the covariant matrix elements of the electromagnetic current between pion states, evaluated in impulse approximation within the Mandelstam approach [30], with all the vertices of the triangle diagram properly dressed. Exploiting a suitable decomposition of the fermionic propagators, one singles out on-shell and instantaneous contributions. The integration over $k^{-}$in the momentum loop of the triangle diagram is performed disregarding the effect of possible singularities of the vertex functions and taking care of only the singularities in the propagators. The validity of this approximation has been tested in the analytic model of Ref. [21] and an essential role played by the choice of a reference frame where $q^{+}>0$ has been shown.

For the photon vertex function, in the processes where a $q \bar{q}$-pair in the odd-parity spin- 1 channel is produced, we use a generalization of the Vector-Meson-dominance approach, built up from the VM Bethe-Salpeter amplitude (phenomenologically determined) and the $\mathrm{VM}$ propagator, enlightening the relation between the hadronic part of the

TABLE III. Vector-meson masses, $M_{n}$, and widths, $\Gamma_{n}$, used in Ref. [26]. The corresponding decay width into $e^{-} e^{+}$pairs, calculated with the VM valence probabilities $P_{q \bar{q} ; n}=\left(1 / \sqrt{2 n+\frac{3}{2}}\right)$ and the oscillator strength $\omega=1.39 \mathrm{GeV}^{2}$, are reported in the sixth column.

\begin{tabular}{lcccccr}
\hline \hline Meson & $M_{n}(\mathrm{MeV})$ & $M_{n}^{\exp }(\mathrm{MeV})[38]$ & $\Gamma_{n}(\mathrm{MeV})$ & $\Gamma_{n}^{\exp }(\mathrm{MeV})[38]$ & $\Gamma_{e^{+} e^{-}}(\mathrm{KeV})$ & $\Gamma_{e^{+} e^{-}}^{\exp }(\mathrm{KeV})[38]$ \\
\hline$\rho(770)$ & 750 & $775.8 \pm 0.5$ & 149 & $146.4 \pm 1.5$ & 6.37 & $7.02 \pm 0.11$ \\
$\rho(1450)$ & 1465 & $1465.0 \pm 25.0$ & 310 & $400 \pm 60$ & 1.61 & $1.47 \pm 0.40$ \\
$\rho(1700)$ & 1723 & $1720.0 \pm 20.0$ & 240 & $250 \pm 100$ & 1.23 & $>0.23 \pm 0.1$ \\
$\rho(2150)$ & 2150 & $2149.0 \pm 17$ & $180^{\mathrm{a}}$ & $363 \pm 50$ & 0.78 & - \\
\hline \hline
\end{tabular}

${ }^{\text {a }}$ The value of $180 \mathrm{MeV}$ for the width of $\rho(2150)$ is the lower bound of the value obtained by Anisovitch et al. quoted in [55]. 
photon valence wave function and the pion electromagnetic form factor.

The expression for the electromagnetic current matrix elements obtained after the $k^{-}$integration are carefully discussed and the different contributions can be immediately interpreted in terms of valence and nonvalence components of the pion and photon wave functions.

As illustrated in Sec. IV, the valence component of the light-front wave function for the pion and for the vector mesons, obtained by a $k^{-}$integration from the corresponding Bethe-Salpeter amplitude (24) and (29) in the valencesector range, fulfills a two-body Schrödinger-like equation with the proper Melosh structure. Therefore, in the valence components of the pion and VM amplitudes, the momentum part is described through the corresponding HLFD wave functions, evaluated in a relativistic model which shows a satisfactory description for the ${ }^{1} S_{0}$ and ${ }^{3} S_{1}$ mesons.

A schematic model is used for the probability, $P_{n, q \bar{q}}$, of the valence component of the vector mesons.

The contribution of the nonvalence component of the photon wave function appears in the timelike region, while the nonvalence component of the pion appears in the spacelike region. The nonvalence contributions of the photon and pion wave functions, relevant for the process under consideration, involve emission/absorption amplitudes, that in principle can be calculated from the valence components of the corresponding particles, and a suitable kernel. However, since our knowledge of this kernel is poor, we use a constant vertex approximation for the emission/absorption amplitudes [18].

To simplify our calculation, we take advantage of the smallness of the pion mass, which is put to zero. Then, only the " $Z$ diagram" survives in the spacelike region.

We point out that, for $m_{\pi}=0$, only the instantaneous terms contribute to the pion form factor. Therefore, in order to evaluate the pion form factor we need the instantaneous vertex functions, which we approximate by the full vertex functions times a constant.

Only a few parameters define our light-front model: the oscillator strength, the constituent quark mass, and the VM meson masses and widths. We use the experimental width and mass for the vector mesons, when available. For the radial excitations above $2.150 \mathrm{GeV}$ we use the masses of theoretical spectrum and a single width as a fitting parameter. It is worth noting that the results are not markedly sensitive upon different pion wave functions, like the asymptotic wave function and the full-model one of Ref. [35]. This could be ascribed to the strong pion binding, that makes the pion wave function similar to its PQCD asymptotic limit [58].
In the spacelike region, the pion electromagnetic form factor is very well described on the whole experimentally explored interval, i.e. up to $q^{2}=-10(\mathrm{GeV} / c)^{2}$. In the timelike region, we find a general agreement up to $10(\mathrm{GeV} / c)^{2}$, except near the experimental dip at $2(\mathrm{GeV} / c)^{2}$.

Our model can be straightforwardly improved in many respects. For instance: (i) more realistic VM wave functions can be used, as the ones of Ref. [52], that take into account, e.g., the $D$-state nature of some of the VM resonances, as $\rho(1700)$; (ii) the introduction of both a dynamical mixing of isospin states and the contribution of $\phi$ meson.

Other improvements, like taking care of the nonvanishing pion mass, or considering a more realistic model for the instantaneous vertices and for the emission/absorption of a pion by a quark, are highly nontrivial.

In summary, our work appears an encouraging step forward in achieving a detailed investigation of important issues, as the light-quark content of the photon valence light-front wave function, through the analysis of the pion electromagnetic form factor in the timelike region. The peculiar feature represented by the smallness of the pion mass is the key point to accomplish such an investigation.

\section{ACKNOWLEDGMENTS}

This work was partially supported by the Brazilian agencies $\mathrm{CNPq}$ and FAPESP and by Ministero dell'Istruzione, dell'Università e della Ricerca. J.P.B.C.M. and T.F. acknowledge the hospitality of the Dipartimento di Fisica, Università di Roma "Tor Vergata" and of Istituto Nazionale di Fisica Nucleare, Sezione Tor Vergata and of Istituto Nazionale di Fisica Nucleare, Sezione Roma I.

\section{APPENDIX A: VECTOR-MESON DECAY CONSTANT}

The vector-meson decay constant $f_{V n}$ of the $n$th state of the vector meson is defined as [16]

$$
\epsilon_{\lambda}^{\mu} \sqrt{2} f_{V, n}=\left\langle 0\left|\bar{q}(0) \gamma^{\mu} q(0)\right| \phi_{n, \lambda}\right\rangle
$$

where $\epsilon_{\lambda}^{\mu}$ are the VM polarization vectors and $\left|\phi_{n, \lambda}\right\rangle$ is the VM state.

Let us begin with the four-dimensional representation of the decay amplitude in terms of the Bethe-Salpeter vertex of the vector meson, and use the plus component of Eq. (A1) and $\lambda=z$ in the rest frame of the vector meson, where $P_{n}^{\mu}=\left[M_{n}, \overrightarrow{0}\right]$ and $\epsilon_{z}^{+}=1$ :

$$
\begin{aligned}
f_{V n}= & -l \frac{N_{c}}{4(2 \pi)^{4}} \int \frac{d k^{-} d k^{+} d \mathbf{k}_{\perp}}{k^{+}\left(P_{n}^{+}-k^{+}\right)} \frac{\Lambda_{n}\left(k, P_{n}\right)}{\left(k^{-}-k_{\mathrm{on}}^{-}+\frac{i \epsilon}{k^{+}}\right)\left(P_{n}^{-}-k^{-}-\left(P_{n}-k\right)_{\mathrm{on}}^{-}+\frac{l \epsilon}{P_{n}^{+}-k^{+}}\right)} \\
& \times \operatorname{Tr}\left[\left(k-\not P_{n}+m\right) \gamma^{+}(k+m)\left[\epsilon_{z}\left(P_{n}\right) \cdot\left[\hat{V}_{n}\left(k, k-P_{n}\right)\right]_{\mathrm{on}}\right]\right] .
\end{aligned}
$$


A factor of $\sqrt{2}$ enters in the denominator of Eq. (A2) from the normalization of the neutral vector meson, i.e., $(u \bar{u}-$ $d \bar{d}\rangle) / \sqrt{2}$. Then integrating over $k^{-}$, with the assumptions on the VM vertex function already presented at the beginning of Sec. III, and taking advantage of the identification of Eqs. (31) and (32), one arrives at a three-dimensional formula for the decay constant where the valence component of the vector-meson wave function appears:

$$
f_{V n}=-\frac{N_{c}}{4(2 \pi)^{3}} \int_{0}^{M_{n}} \frac{d k^{+} d \mathbf{k}_{\perp}}{k^{+}\left(M_{n}-k^{+}\right)} \psi_{n}\left(k^{+}, \mathbf{k}_{\perp} ; M_{n}, \mathbf{0}_{\perp}\right) \operatorname{Tr}\left[\left(\not k-\not P_{n}+m\right) \gamma^{+}(\not k+m)\left(\notin_{z}-\frac{\left(k_{\mathrm{on}}-\left(P_{n}-k\right)_{\mathrm{on}}\right) \cdot \boldsymbol{\epsilon}_{z}}{M_{0}\left(k^{+}, \mathbf{k}_{\perp} ; P_{n}^{+}, \mathbf{P}_{n \perp}\right)+2 m}\right)\right] .
$$

Evaluating the trace in Eq. (A3) the final expression of the decay constant is

$$
\begin{aligned}
f_{V n}= & -\frac{N_{c}}{8 \pi^{3}} \int_{0}^{M_{n}} \frac{d k^{+} d \mathbf{k}_{\perp}}{k^{+}\left(M_{n}-k^{+}\right)} \psi_{n}\left(k^{+}, \mathbf{k}_{\perp} ; M_{n}, \mathbf{0}_{\perp}\right)\left[m^{2}-k_{\mathrm{on}} \cdot\left(P_{n}-k\right)_{\mathrm{on}}-\left(P_{n}-k\right)_{\mathrm{on}, z} k^{+}+k_{\mathrm{on}, z}\left(M_{n}-k^{+}\right)\right. \\
& \left.+m\left(2 k^{+}-M_{n}\right) \frac{\left(k_{\mathrm{on}}-\left(P_{n}-k\right)_{\mathrm{on}}\right)_{z}}{M_{0}+2 m}\right],
\end{aligned}
$$

where $M_{0}^{2}=\left(\mathbf{k}_{\perp}^{2}+m^{2}\right) /(x(1-x))$ with $x=k^{+} / M_{n}$.

From the vector-meson decay constant one gets the decay width to $e^{+} e^{-}$as [8]

$$
\Gamma_{e^{+} e^{-}}=\frac{8 \pi \alpha^{2}}{3} \frac{f_{V n}^{2}}{M_{n}^{3}}
$$

where $\alpha$ is the fine structure constant.

\section{APPENDIX B: CURRENT CONSERVATION}

Let us define the four quantities

$$
\mathcal{V}_{n}^{\mu}=\bar{V}_{n}^{\mu} \frac{q \cdot P_{n}}{M_{n}^{2}}-\frac{P_{n}^{\mu}}{M_{n}^{2}} q \cdot \bar{V}_{n}
$$

where, see Eq. (16) of Sec. II,

$$
\bar{V}_{n}^{\mu}=\left[\hat{V}_{n}^{\mu}(k, k-q)\right]_{\mathrm{on}}=\gamma^{\mu}-\frac{k_{\mathrm{on}}^{\mu}-(q-k)_{\mathrm{on}}^{\mu}}{M_{0}+2 m} .
$$

One can immediately verify that

$$
q \cdot V_{n}=0
$$

Since the vector-meson propagator [45] is given by

$$
D_{\nu}^{\mu}=\left[-g_{\nu}^{\mu}+\frac{q^{\mu} q_{\nu}}{M_{n}^{2}}\right] \frac{1}{\left[q^{2}-M_{n}^{2}+\imath M_{n} \tilde{\Gamma}_{n}\left(q^{2}\right)\right]}
$$

a possible conserved photon- $(q \bar{q})$ dressed vertex can be defined as follows:

$$
\mathcal{J}^{\mu}(k, q)=\sum_{n} \mathcal{J}_{n}^{\mu}(k, q)
$$

with

$$
\begin{aligned}
\mathcal{J}_{n}^{\mu}(k, q)= & \sqrt{2}\left[-g^{\mu}{ }_{\nu}+\frac{q^{\mu} q_{\nu}}{M_{n}^{2}}\right] \mathcal{V}_{n}^{\nu} \Lambda_{n}(k, q) \\
& \times \frac{f_{V n}}{\left[q^{2}-M_{n}^{2}+\imath M_{n} \tilde{\Gamma}_{n}\left(q^{2}\right)\right]} \\
= & -\sqrt{2} \mathcal{V}_{n}^{\mu} \Lambda_{n}(k, q) \frac{f_{V n}}{\left[q^{2}-M_{n}^{2}+\imath M_{n} \tilde{\Gamma}_{n}\left(q^{2}\right)\right]} .
\end{aligned}
$$

Indeed it is straightforward to show that $q \cdot \mathcal{J}(k, q)=0$, since one has $q \cdot \mathcal{V}_{n}=0$.

For each term $\mathcal{J}_{n}^{\mu}$, let us consider the reference frame where $q^{+}=M_{n}>0$ and $\mathbf{q}_{\perp}=0$ (see Sec. VII for the possibility to use different reference frame for different terms in the sum). In this reference frame one has $q^{-}=$ $q^{2} / M_{n}$ and then

$$
q \cdot \bar{V}_{n}=\frac{1}{2}\left[q^{-} \bar{V}_{n}^{+}+q^{+} \bar{V}_{n}^{-}\right]=\frac{1}{2}\left[\bar{V}_{n}^{+} q^{2} / M_{n}+M_{n} \bar{V}_{n}^{-}\right],
$$

$$
q \cdot P_{n}=\frac{1}{2}\left[q^{2}+M_{n}^{2}\right] .
$$

Therefore one obtains

$$
\mathcal{V}_{n}^{+}=\frac{1}{2}\left[\left(\frac{q^{2}}{M_{n}^{2}}+1\right) \bar{V}_{n}^{+}-\frac{1}{M_{n}}\left(\bar{V}_{n}^{+} q^{2} / M_{n}+M_{n} \bar{V}_{n}^{-}\right)\right]=\bar{V}_{n, z}
$$

and in conclusion we have

$$
\mathcal{J}_{n}^{+}(k, q)=-\sqrt{2} \bar{V}_{n, z} \Lambda_{n}(k, q) \frac{f_{V n}}{\left[q^{2}-M_{n}^{2}+\imath M_{n} \tilde{\Gamma}_{n}\left(q^{2}\right)\right]}
$$

If in Eq. (B1) the quantity $\bar{V}_{n}^{\mu}$ is replaced by 


$$
\hat{V}_{n}^{\mu}\left(k, k-P_{n}\right)=\gamma^{\mu}-\frac{k^{\mu}+k^{\prime \mu}}{M_{n}+2 m}
$$

as defined in Eq. (14), then the current $\mathcal{J}^{\mu}(k, q)$ is a fourvector.

Let us note that, if in Eq. (B10) the momentum component of the VM vertex function $\Lambda_{n}(k, q)$ is taken at the VM pole, then the plus component of the current $\mathcal{J}^{+}(k, q)$ coincides with the one used in our calculations [see Eq. (G3)].

\section{APPENDIX C: SUBTRACTION OF THE BARE TERM IN THE PHOTON LF VERTEX}

Let us calculate explicitly the contribution to the current of a $\gamma^{\mu}$ bare term in the case of massless pions, in collinear kinematics $\left(\mathbf{q}_{\perp}=0, P_{\pi}^{+}=0, \mathbf{P}_{\pi \perp}=0, P_{\bar{\pi}}^{+}=q^{+}, \mathbf{P}_{\bar{\pi} \perp}=\right.$ $0)$. As discussed at length in Sec. VII and VIII, only the instantaneous term of the spectator constituent propagator can give a contribution in the limit of a vanishing pion mass. Therefore one has for the plus (minus) component

$$
\begin{aligned}
\delta j^{ \pm}= & -2 \imath \frac{e}{(2 \pi)^{4}} \frac{m^{2}}{f_{\pi}^{2}} N_{c} \int d^{4} k \Lambda_{\bar{\pi}}\left(k-P_{\pi}, P_{\bar{\pi}}\right) \bar{\Lambda}_{\pi}\left(k, P_{\pi}\right) \operatorname{Tr}\left[\gamma^{5} \frac{\gamma^{+}}{2 k^{+}} \gamma^{5}[k-\not 1+m] \gamma^{ \pm}[k+m]\right] \\
& \times \frac{1}{\left(k^{2}-m^{2}+\imath \epsilon\right)\left((k-q)^{2}-m^{2}+\imath \epsilon\right)}
\end{aligned}
$$

In the adopted frame, i.e. $\mathbf{q}_{\perp}=0$, the current conservation for the bare term reads

$$
\delta j^{+} q^{-}+\delta j^{-} q^{+}=0
$$

Let us note that in [19,21], where simple Ansätze for the vertex functions of photon and pion, respectively, are assumed, the bare term fulfills the current conservation, since it is possible to take into account the full analytical structure of the considered vertex functions.
In the present approach, the contributions from the singularities of the meson vertex functions are neglected in the integration over $k^{-}$, namely, only contributions from the propagator poles are retained. Therefore in Eq. (C1), let us (i) perform the trace and the integration on $k^{-}$taking into account only the propagators poles, and (ii) use the identifications in Eqs. (26), (28), and (39) with $m_{\pi}=0$. Then we have the following approximation for $\delta j^{+}$, viz

$$
\delta j_{\text {app }}^{+}=-\frac{4 e N_{c}}{(2 \pi)^{3}} \int_{0}^{q^{+}} \frac{d k^{+}}{k^{+}} \int d \mathbf{k}_{\perp} \overline{\mathcal{D}}_{\pi} \frac{q^{+}}{\left[M_{0}^{2}\left(k^{+}, \mathbf{k}_{\perp} ; q^{+}, \mathbf{0}_{\perp}\right)-q^{2}+\imath \epsilon\right]} \psi_{\bar{\pi}}\left(k^{+}, \mathbf{k}_{\perp} ; P_{\bar{\pi}}^{+}, \mathbf{0}_{\perp}\right) \frac{M_{0}^{2}\left(k^{+}, \mathbf{k}_{\perp} ; P_{\bar{\pi}}^{+}, \mathbf{0}_{\perp}\right)}{P_{\bar{\pi}}^{+}} .
$$

After evaluating $\delta j_{\text {app }}^{-}$, within the same assumptions used for $\delta j_{\text {app }}^{+}$, the four divergence of the current reads as follows:

$$
\delta j_{\text {app }}^{+} q^{-}+\delta j_{\text {app }}^{-} q^{+}=\frac{4 e N_{c}}{(2 \pi)^{3}} \frac{1}{q^{+}} \int_{0}^{q^{+}} \frac{d k^{+}}{k^{+}} \int d \mathbf{k}_{\perp} \overline{\mathcal{D}}_{\pi} M_{0}^{2}\left(k^{+}, \mathbf{k}_{\perp} ; q^{+}, \mathbf{0}_{\perp}\right) \psi_{\bar{\pi}}\left(k^{+}, \mathbf{k}_{\perp} ; P_{\bar{\pi}}^{+}=q^{+}, \mathbf{0}_{\perp}\right) \frac{\left(2 q^{+}-k^{+}\right)}{\left(q^{+}-k^{+}\right)} .
$$

Since in our model (i) the pion wave function at large momentum has the asymptotic form given by Eq. (82), which decays as $1 /\left|\mathbf{k}_{\perp}\right|^{2}$, and (ii) $\mathcal{D}_{\pi}$ is taken as a constant, then the integrals in Eqs. (C2) and (C3) are ultraviolet divergent. In principle, a regularization of the integrals could be obtained by using a momentum dependent approximation for $\mathcal{D}_{\pi}$, and a different approximation for the pion wave function, beyond the present model.

Therefore, in the model of the present paper, where a massless pion is assumed, the contributions from the singularities of the pion vertex function are disregarded and $\mathcal{D}_{\pi}$ is taken as a constant, we give out the bare term in the photon-vertex function.

\section{APPENDIX D: INTEGRATION OF THE TRIANGLE DIAGRAM OVER $\boldsymbol{k}^{-}$}

The triangle diagram (Fig. 1) is integrated over $k^{-}$with the assumptions stated at the beginning of Sec. III.

To make clear the discussion of the $k^{-}$integration, it is helpful to first separate instantaneous and noninstantaneous contributions, using the decomposition of the Dirac propagator given in Eq. (2). Indeed this decomposition is useful to have a better control on possible divergences both in $k^{-}$and in $k^{+}$integrations. In particular, as already mentioned, it should be pointed out the tight relation between the instantaneous terms and the so-called zero 
modes, where $k^{+}=0$. We assume that the behavior of the functions $\Lambda(k, P)$ in $k^{+}$is able to regularize the divergences at the $k^{+}$end points [59]. At the end of Sec. IX it is shown that this is actually the case with our model for the meson vertex functions.

Since three propagators are present in Eq. (3), one has a total of eight contributions. The contribution with three instantaneous terms vanishes because of the property $\gamma^{+} \gamma^{+}=0$, since the combination $\gamma^{+} \gamma^{5} \gamma^{+}$appears. Also the three contributions with two instantaneous terms vanish, as a consequence of our assumptions on $\Lambda(k, P)$. Indeed, only a single pole from the propagators is present in these contributions. Then, since we assume that the functions $\Lambda(k, P)$ go to zero for $\left|k^{-}\right| \rightarrow \infty$ and disregard their singularities, we can perform the integration in the $k^{-}$ complex plane closing the contour in the semiplane where no singularity in the propagators is present and we obtain a null result. Moreover, two of these contributions with two instantaneous terms are also identically vanishing because of the presence of the combination $\gamma^{+} \gamma^{5} \gamma^{+}$.

Therefore we are left with four contributions: three contributions with one instantaneous term only and one contribution with no instantaneous term.

To evaluate the triangle diagram we treat separately the timelike case and the spacelike case.

\section{Timelike case}

In the timelike case, one has $q^{\mu}=P_{\pi}^{\mu}+P_{\bar{\pi}}^{\mu}$, and $q^{+}>$ 0 . Equation (3) written in light-front variables becomes (the Jacobian for the transformation to the light-front variables is $1 / 2)$ :

$$
\begin{aligned}
j^{\mu}= & -l \frac{e}{(2 \pi)^{4}} \frac{m^{2}}{f_{\pi}^{2}} N_{c} \int \frac{d k^{-} d k^{+} d \mathbf{k}_{\perp}}{\left(k^{+}-P_{\pi}^{+}\right) k^{+}\left(k^{+}-q^{+}\right)} \operatorname{Tr}\left[\mathcal{O}^{\mu}\right] \\
& \times \frac{\Lambda_{\bar{\pi}}\left(k-P_{\pi}, P_{\bar{\pi}}\right) \bar{\Lambda}_{\pi}\left(k, P_{\pi}\right)}{\left(k^{-}-k_{\mathrm{on}}^{-}+\frac{l \epsilon}{k^{+}}\right)\left(k^{-}-q^{-}-(k-q)_{\mathrm{on}}^{-}+\frac{l \epsilon}{k^{+}-q^{+}}\right)\left(k^{-}-P_{\pi}^{-}-\left(k-P_{\pi}\right)_{\mathrm{on}}^{-}+\frac{l \epsilon}{k^{+}-P_{\pi}^{+}}\right)} .
\end{aligned}
$$

The on-mass-shell values of the minus-components of the momenta in Eq. (D1) are given by

$$
k_{\mathrm{on}}^{-}=\frac{\mathbf{k}_{\perp}^{2}+m^{2}}{k^{+}}, \quad(k-q)_{\mathrm{on}}^{-}=\frac{(\mathbf{k}-\mathbf{q})_{\perp}^{2}+m^{2}}{k^{+}-q^{+}}, \quad\left(k-P_{\pi}\right)_{\mathrm{on}}^{-}=\frac{\left(\mathbf{k}-\mathbf{P}_{\pi}\right)_{\perp}^{2}+m^{2}}{k^{+}-P_{\pi}^{+}},
$$

and the operator $\mathcal{O}^{\mu}$ is defined as follows:

$$
\mathcal{O}^{\mu}=\left(\not k-\not P_{\pi}+m\right) \gamma^{5}(\not k-\not 1+m) \Gamma^{\mu}(k, q)(\not k+m) \gamma^{5}
$$

Let us perform the decomposition of the propagators in instantaneous and in on-shell parts [see Eq. (2)], as discussed at the beginning of this Appendix. Then Eq. (D1) becomes

$$
j^{\mu}=\mathcal{J}_{\mathrm{on}}^{\mu}+\mathcal{J}_{1}^{\mu}+\mathcal{J}_{2}^{\mu}+\mathcal{J}_{3}^{\mu},
$$

where $\mathcal{J}_{\text {on }}^{\mu}$ represents the on-shell contribution and $\mathcal{J}_{i}^{\mu}(i=1,2,3)$ represent the contributions with one instantaneous term. Then we have

$$
\mathcal{J}_{\mathrm{on}}^{\mu}=-\imath \frac{e}{(2 \pi)^{4}} \frac{m^{2}}{f_{\pi}^{2}} N_{c} \int \frac{d k^{-} d k^{+} d \mathbf{k}_{\perp}}{\left(k^{+}-P_{\pi}^{+}\right) k^{+}\left(k^{+}-q^{+}\right)} \Lambda_{\bar{\pi}}\left(k-P_{\pi}, P_{\bar{\pi}}\right) \bar{\Lambda}_{\pi}\left(k, P_{\pi}\right) \mathcal{T}_{\mathrm{on}}^{\mu}
$$

and

$$
\mathcal{J}_{i}^{\mu}=-\imath \frac{e}{(2 \pi)^{4}} \frac{m^{2}}{f_{\pi}^{2}} N_{c} \int \frac{d k^{-} d k^{+} d \mathbf{k}_{\perp}}{\left(k^{+}-P_{\pi}^{+}\right) k^{+}\left(k^{+}-q^{+}\right)} \Lambda_{\bar{\pi}}\left(k-P_{\pi}, P_{\bar{\pi}}\right) \bar{\Lambda}_{\pi}\left(k, P_{\pi}\right) \mathcal{T}_{i}^{\mu},
$$

where

$$
\begin{gathered}
\mathcal{T}_{\mathrm{on}}^{\mu}=\frac{\operatorname{Tr}\left[\left[\left(k-\not p_{\pi}\right)_{\mathrm{on}}+m\right] \gamma^{5}\left[(k-\not)_{\mathrm{on}}+m\right] \Gamma^{\mu}(k, q)\left(k_{\mathrm{on}}+m\right) \gamma^{5}\right]}{\left(k^{-}-k_{\mathrm{on}}^{-}+\frac{l \epsilon}{k^{+}}\right)\left(k^{-}-q^{-}-(k-q)_{\mathrm{on}}^{-}+\frac{l \epsilon}{k^{+}-q^{+}}\right)\left(k^{-}-P_{\pi}^{-}-\left(k-P_{\pi}\right)_{\mathrm{on}}^{-}+\frac{l \epsilon}{k^{+}-P_{\pi}^{+}}\right)}, \\
\mathcal{T}_{1}^{\mu}=\frac{\operatorname{Tr}\left[\gamma^{+} \gamma^{5}\left[(k-\not)_{\mathrm{on}}+m\right] \Gamma^{\mu}(k, q)\left(k_{\mathrm{on}}+m\right) \gamma^{5}\right]}{2\left(k^{-}-k_{\mathrm{on}}^{-}+\frac{l \epsilon}{k^{+}}\right)\left(k^{-}-q^{-}-(k-q)_{\mathrm{on}}^{-}+\frac{l \epsilon}{k^{+}-q^{+}}\right)},
\end{gathered}
$$




$$
\begin{gathered}
\mathcal{T}_{2}^{\mu}=\frac{\operatorname{Tr}\left[\left[\left(k-\not P_{\pi}\right)_{\mathrm{on}}+m\right] \gamma^{5} \gamma^{+} \Gamma^{\mu}(k, q)\left(k_{\mathrm{on}}+m\right) \gamma^{5}\right]}{2\left(k^{-}-k_{\mathrm{on}}^{-}+\frac{l \epsilon}{k^{+}}\right)\left(k^{-}-P_{\pi}^{-}-\left(k-P_{\pi}\right)_{\mathrm{on}}^{-}+\frac{l \epsilon}{k^{+}-P_{\pi}^{+}}\right)}, \\
\mathcal{T}_{3}^{\mu}=\frac{\operatorname{Tr}\left[\left[\left(k-\not P_{\pi}\right)_{\mathrm{on}}+m\right] \gamma^{5}\left[(k-\not)_{\mathrm{on}}+m\right] \Gamma^{\mu}(k, q) \gamma^{+} \gamma^{5}\right]}{2\left(k^{-}-q^{-}-(k-q)_{\mathrm{on}}^{-}+\frac{l \epsilon}{k^{+}-q^{+}}\right)\left(k^{-}-P_{\pi}^{-}-\left(k-P_{\pi}\right)_{\mathrm{on}}^{-}+\frac{l \epsilon}{k^{+}-P_{\pi}^{+}}\right)} .
\end{gathered}
$$

In Eqs. (D5) and (D6) the three propagators of the triangle diagram generate three poles:

$$
k_{(1)}^{-}=k_{\mathrm{on}}^{-}-\frac{l \epsilon}{k^{+}}, \quad k_{(2)}^{-}=q^{-}+(k-q)_{\mathrm{on}}^{-}-\frac{l \epsilon}{k^{+}-q^{+}}, \quad k_{(3)}^{-}=P_{\pi}^{-}+\left(k-P_{\pi}\right)_{\mathrm{on}}^{-}-\frac{l \epsilon}{k^{+}-P_{\pi}^{+}} .
$$

Within our assumptions on the vertex functions $\Lambda(k, P)$, if $k^{+}<0$ there are no poles in the lower complex semiplane of $k^{-}$[cf. Eq. (D11)]. Therefore, if the $k^{-}$integration is performed by closing the contour of integration in the lower semiplane, a vanishing result is obtained. Furthermore, if $k^{+}>q^{+}$, there are no poles in the upper complex semiplane and a vanishing result is obtained by closing the contour in the upper semiplane. Then, the integrals (D5) and (D6) have contributions only for $k^{+}$in the range $0<k^{+}<q^{+}$. The integration range can be decomposed in two intervals, $0<k^{+}<P_{\pi}^{+}$[diagram (a) of Fig. 2] and $P_{\pi}^{+}<k^{+}<q^{+}$(diagram (b) of Fig. 2). In the first one, if the $k^{-}$integration contour is closed in the lower semiplane, only the pole $k_{(1)}^{-}$falls within the integration contour, while in the second one, if the integration contour is closed in the upper semiplane, only the pole $k_{(2)}^{-}$falls within the integration contour. Then in the range $0<k^{+}<$ $P_{\pi}^{+}$one has contributions from $\mathcal{J}_{\text {on }}^{\mu}, \mathcal{J}_{1}^{\mu}$ and $\mathcal{J}_{2}^{\mu}$, while in the range $P_{\pi}^{+}<k^{+}<q^{+}$one has contributions from $\mathcal{J}_{\text {on }}^{\mu}$, $\mathcal{J}_{1}^{\mu}$ and $\mathcal{J}_{3}^{\mu}$.

For a $q \bar{q}$ system, with four-momentum $P^{\mu} \equiv\left\{\left(M^{2}+\right.\right.$ $\left.\left.\left|\mathbf{P}_{\perp}\right|^{2}\right) / P^{+}, P^{+}, \mathbf{P}_{\perp}\right\}$, the square free mass $M_{0}^{2}\left(k^{+}, \mathbf{k}_{\perp} ; P^{+}, \mathbf{P}_{\perp}\right)$ can be written as follows:

$M_{0}^{2}\left(k^{+}, \mathbf{k}_{\perp} ; P^{+}, \mathbf{P}_{\perp}\right)=\frac{\mathbf{k}_{\perp}^{2}+m^{2}}{x}+\frac{(\mathbf{P}-\mathbf{k})_{\perp}^{2}+m^{2}}{1-x}-\mathbf{P}_{\perp}^{2}$,

where $\left(k^{+}, \mathbf{k}_{\perp}\right)$ and $\left(P^{+}-k^{+}, \mathbf{P}_{\perp}-\mathbf{k}_{\perp}\right)$ are the LF momenta of the quarks, and $x=k^{+} / P^{+}$, with $0 \leq x \leq 1$. Using this definition of the free mass, the following equations hold:

$$
\begin{aligned}
& \frac{1}{\left(P^{-}-(P-k)_{\mathrm{on}}^{-}-k_{\mathrm{on}}^{-}\right)}=\frac{P^{+}}{\left(M^{2}+\mathbf{P}_{\perp}^{2}-\frac{(\mathbf{P}-\mathbf{k})_{\perp}^{2}+m^{2}}{(1-x)}-\frac{\mathbf{k}_{\perp}^{2}+m^{2}}{x}\right)}=\frac{P^{+}}{\left(M^{2}-M_{0}^{2}\left(k^{+}, \mathbf{k}_{\perp} ; P^{+}, \mathbf{P}_{\perp}\right)\right)} \\
& \frac{1}{\left[P^{\prime-}-\left(P^{\prime}-(k-P)\right)_{\mathrm{on}}^{-}-(k-P)_{\mathrm{on}}^{-}\right]}=\frac{P^{\prime+}}{\left(M^{2}+\mathbf{P}_{\perp}^{\prime 2}-\frac{(\mathbf{P}-\mathbf{k})_{\perp}^{2}+m^{2}}{x^{\prime}}-\frac{\left(\mathbf{P}^{\prime}-(\mathbf{k}-\mathbf{P})\right)_{\perp}^{2}+m^{2}}{\left(1-x^{\prime}\right)}\right)} \\
& =\frac{P^{\prime+}}{\left[M^{2}-M_{0}^{2}\left((k-P)^{+},(\mathbf{k}-\mathbf{P})_{\perp} ; P^{\prime+}, \mathbf{P}_{\perp}^{\prime}\right)\right]},
\end{aligned}
$$

where $x^{\prime}=\left(k^{+}-P^{+}\right) / P^{\prime+}$.

Then, performing the $k^{-}$integration and using Eqs. (D13) and (D14) from Eq. (D4) we obtain

$$
j^{\mu}=\frac{e}{(2 \pi)^{3}} \frac{m^{2}}{f_{\pi}^{2}} N_{c} \int_{0}^{q^{+}} \frac{d k^{+} d \mathbf{k}_{\perp}}{\left(k^{+}-P_{\pi}^{+}\right) k^{+}\left(q^{+}-k^{+}\right)}\left\{\Theta\left(P_{\pi}^{+}-k^{+}\right) I_{1}^{\mu}+\Theta\left(k^{+}-P_{\pi}^{+}\right) I_{2}^{\mu}\right\} .
$$

The quantities $I_{1}^{\mu}$ and $I_{2}^{\mu}$ in Eq. (D15) are defined as follows:

$$
\begin{gathered}
I_{1}^{\mu}=\left[\bar{\Lambda}_{\pi}\left(k, P_{\pi}\right) \Lambda_{\bar{\pi}}\left(k-P_{\pi}, P_{\bar{\pi}}\right)\right]_{k^{-}=k_{\mathrm{on}}^{-}}\left[T_{\mathrm{on},(1)}^{\mu}+T_{1,(1)}^{\mu}+T_{2,(1)}^{\mu}\right], \\
I_{2}^{\mu}=\left[\bar{\Lambda}_{\pi}\left(k, P_{\pi}\right) \Lambda_{\bar{\pi}}\left(k-P_{\pi}, P_{\bar{\pi}}\right)\right]_{k^{-}=q^{-}+(k-q)_{\mathrm{on}}^{-}}\left[T_{\mathrm{on},(2)}^{\mu}+T_{1,(2)}^{\mu}+T_{3,(2)}^{\mu}\right],
\end{gathered}
$$

where

$$
T_{\mathrm{on},(1)}^{\mu}=q^{+} P_{\pi}^{+} \frac{\operatorname{Tr}\left[\left[\left(k-\not P_{\pi}\right)_{\mathrm{on}}+m\right] \gamma^{5}\left[(k-\not)_{\mathrm{on}}+m\right] \Gamma^{\mu}(1)\left(k_{\mathrm{on}}+m\right) \gamma^{5}\right]}{\left[M_{0}^{2}\left(k^{+}, \mathbf{k}_{\perp} ; q^{+}, \mathbf{q}_{\perp}\right)-q^{2}-\imath \epsilon\right]\left[M_{0}^{2}\left(k^{+}, \mathbf{k}_{\perp} ; P_{\pi}^{+}, \mathbf{P}_{\pi \perp}\right)-m_{\pi}^{2}\right]}
$$




$$
\begin{gathered}
T_{\mathrm{on},(2)}^{\mu}=\frac{q^{+} P_{\bar{\pi}}^{+} \operatorname{Tr}\left[\left[\left(k-\not P_{\pi}\right)_{\mathrm{on}}+m\right] \gamma^{5}\left[(k-\not)_{\mathrm{on}}+m\right] \Gamma^{\mu}(2)\left(k_{\mathrm{on}}+m\right) \gamma^{5}\right]}{\left[M_{0}^{2}\left(k^{+}, \mathbf{k}_{\perp} ; q^{+}, \mathbf{q}_{\perp}\right)-q^{2}-\imath \epsilon\right]\left[m_{\pi}^{2}-M_{0}^{2}\left(\left(k^{+}-P_{\pi}^{+}\right),\left(\mathbf{k}-\mathbf{P}_{\pi}\right)_{\perp} ; P_{\bar{\pi}}^{+}, \mathbf{P}_{\bar{\pi} \perp}\right)\right]}, \\
T_{1,(i)}^{\mu}=q^{+} \frac{\operatorname{Tr}\left[\gamma^{+} \gamma^{5}\left[(\not k-\not)_{\mathrm{on}}+m\right] \Gamma^{\mu}(i)\left(k_{\mathrm{on}}+m\right) \gamma^{5}\right]}{2\left[M_{0}^{2}\left(k^{+}, \mathbf{k}_{\perp} ; q^{+}, \mathbf{q}_{\perp}\right)-q^{2}-\imath \epsilon\right]} \quad(i=1,2), \\
T_{2,(1)}^{\mu}=P_{\pi}^{+} \frac{\operatorname{Tr}\left[\left[\left(k-\not p_{\pi}\right)_{\mathrm{on}}+m\right] \gamma^{5} \gamma^{+} \Gamma^{\mu}(1)\left(k_{\mathrm{on}}+m\right) \gamma^{5}\right]}{2\left[M_{0}^{2}\left(k^{+}, \mathbf{k}_{\perp} ; P_{\pi}^{+}, \mathbf{P}_{\pi \perp}\right)-m_{\pi}^{2}\right]}, \\
T_{3,(2)}^{\mu}=P_{\bar{\pi}}^{+} \frac{\operatorname{Tr}\left[\left[\left(k-\not P_{\pi}\right)_{\mathrm{on}}+m\right] \gamma^{5}\left[(k-\not)_{\mathrm{on}}+m\right] \Gamma^{\mu}(2) \gamma^{+} \gamma^{5}\right]}{2\left[M_{0}^{2}\left(\left(k^{+}-P_{\pi}^{+}\right),\left(\mathbf{k}-\mathbf{P}_{\pi}\right)_{\perp} ; P_{\bar{\pi}}^{+}, \mathbf{P}_{\bar{\pi} \perp}\right)-m_{\pi}^{2}\right]},
\end{gathered}
$$

with

$$
\Gamma^{\mu}(i)=\Gamma^{\mu}\left(k^{+}, \mathbf{k}_{\perp}, k^{-}=k_{(i)}^{-}, q\right) \quad(i=1,2)
$$

\section{Spacelike case}

In the spacelike case one has to carry out a similar detailed analysis. In particular now one has $P_{\pi^{\prime}}^{\mu}=P_{\pi}^{\mu}+q^{\mu}$. Then the expression for the triangle diagram can be obtained from Eq. (3) replacing $-P_{\pi}^{\mu}$ with $P_{\pi}^{\mu}, \bar{\pi}$ with $\pi^{\prime}$ and the pion vertices $\bar{\Lambda}_{\pi}\left(k, P_{\pi}\right)$ and $\Lambda_{\bar{\pi}}\left(k-P_{\pi}, P_{\bar{\pi}}\right)$ with $\Lambda_{\pi}\left(-k, P_{\pi}\right)$ and $\bar{\Lambda}_{\pi^{\prime}}\left(k+P_{\pi}, P_{\pi^{\prime}}\right)$, respectively:

$$
\begin{aligned}
j^{\mu}= & -\imath 2 e \frac{m^{2}}{f_{\pi}^{2}} N_{c} \int \frac{d^{4} k}{(2 \pi)^{4}} \operatorname{Tr}\left[S\left(k+P_{\pi}\right) \gamma^{5} S(k-q) \Gamma^{\mu}(k, q) S(k) \gamma^{5}\right] \bar{\Lambda}_{\pi^{\prime}}\left(k+P_{\pi}, P_{\pi^{\prime}}\right) \Lambda_{\pi}\left(-k, P_{\pi}\right) \\
= & -\imath \frac{e}{(2 \pi)^{4}} \frac{m^{2}}{f_{\pi}^{2}} N_{c} \int \frac{d k^{-} d k^{+} d \mathbf{k}_{\perp}}{\left(k^{+}+P_{\pi}^{+}\right) k^{+}\left(k^{+}-q^{+}\right)} \operatorname{Tr}\left[\mathcal{O}^{\prime \mu}\right] \\
& \times \frac{\bar{\Lambda}_{\pi^{\prime}}\left(k+P_{\pi}, P_{\pi^{\prime}}\right) \Lambda_{\pi}\left(-k, P_{\pi}\right)}{\left(k^{-}-k_{\mathrm{on}}^{-}+\frac{l \epsilon}{k^{+}}\right)\left(k^{-}-q^{-}-(k-q)_{\mathrm{on}}^{-}+\frac{l \epsilon}{k^{+}-q^{+}}\right)\left(k^{-}+P_{\pi}^{-}-\left(k+P_{\pi}\right)_{\mathrm{on}}^{-}+\frac{\iota \epsilon}{k^{+}+P_{\pi}^{+}}\right)},
\end{aligned}
$$

where

$$
\mathcal{O}^{\prime \mu}=\left(\not k+\not p_{\pi}+m\right) \gamma^{5}(\not k-\not 1+m) \Gamma^{\mu}(k, q)(\not k+m) \gamma^{5} .
$$

As in the timelike case, let us decompose the propagators in on-shell and instantaneous parts. Then Eq. (D24) becomes

$$
j^{\mu}=\mathcal{J}_{\mathrm{on}}^{\prime \mu}+\mathcal{J}_{1}^{\prime \mu}+\mathcal{J}_{2}^{\prime \mu}+\mathcal{J}_{3}^{\prime \mu}
$$

where $\mathcal{J}_{\text {on }}^{\prime \mu}$ represents the on-shell contribution and $\mathcal{J}_{i}^{\prime \mu}(i=1,2,3)$ represent the contributions with one instantaneous term. Then we have

$$
\mathcal{J}_{\mathrm{on}}^{\prime \mu}=-\imath \frac{e}{(2 \pi)^{4}} \frac{m^{2}}{f_{\pi}^{2}} N_{c} \int \frac{d k^{-} d k^{+} d \mathbf{k}_{\perp}}{\left(k^{+}+P_{\pi}^{+}\right) k^{+}\left(k^{+}-q^{+}\right)} \bar{\Lambda}_{\pi^{\prime}}\left(k+P_{\pi}, P_{\pi^{\prime}}\right) \Lambda_{\pi}\left(-k, P_{\pi}\right) \mathcal{T}_{\mathrm{on}}^{\prime \mu}
$$

and

$$
\mathcal{J}_{i}^{\prime \mu}=-\imath \frac{e}{(2 \pi)^{4}} \frac{m^{2}}{f_{\pi}^{2}} N_{c} \int \frac{d k^{-} d k^{+} d \mathbf{k}_{\perp}}{\left(k^{+}+P_{\pi}^{+}\right) k^{+}\left(k^{+}-q^{+}\right)} \bar{\Lambda}_{\pi^{\prime}}\left(k+P_{\pi}, P_{\pi^{\prime}}\right) \Lambda_{\pi}\left(-k, P_{\pi}\right) \mathcal{T}_{i}^{\prime \mu}
$$

where 


$$
\begin{gathered}
\mathcal{T}_{\text {on }}^{\prime \mu}=\frac{\operatorname{Tr}\left[\left[\left(k+\not P_{\pi}\right)_{\mathrm{on}}+m\right] \gamma^{5}\left[(k-\not)_{\mathrm{on}}+m\right] \Gamma^{\mu}(k, q)\left(k_{\mathrm{on}}+m\right) \gamma^{5}\right]}{\left(k^{-}-k_{\mathrm{on}}^{-}+\frac{l \epsilon}{k^{+}}\right)\left(k^{-}-q^{-}-(k-q)_{\mathrm{on}}^{-}+\frac{l \epsilon}{k^{+}-q^{+}}\right)\left(k^{-}+P_{\pi}^{-}-\left(k+P_{\pi}\right)_{\mathrm{on}}^{-}+\frac{l \epsilon}{k^{+}+P_{\pi}^{+}}\right)} \\
\mathcal{T}_{1}^{\prime \mu}=\mathcal{T}_{1}^{\mu}=\frac{\operatorname{Tr}\left[\gamma^{+} \gamma^{5}\left[(k-\not)_{\mathrm{on}}+m\right] \Gamma^{\mu}(k, q)\left(k_{\mathrm{on}}+m\right) \gamma^{5}\right]}{2\left(k^{-}-k_{\mathrm{on}}^{-}+\frac{l \epsilon}{k^{+}}\right)\left(k^{-}-q^{-}-(k-q)_{\mathrm{on}}^{-}+\frac{l \epsilon}{k^{+}-q^{+}}\right)} \\
\mathcal{T}_{2}^{\prime \mu}=\frac{\operatorname{Tr}\left[\left[\left(k+\not P_{\pi}\right)_{\mathrm{on}}+m\right] \gamma^{5} \gamma^{+} \Gamma^{\mu}(k, q)\left(k_{\mathrm{on}}+m\right) \gamma^{5}\right]}{2\left(k^{-}-k_{\mathrm{on}}^{-}+\frac{l \epsilon}{k^{+}}\right)\left(k^{-}+P_{\pi}^{-}-\left(k+P_{\pi}\right)_{\mathrm{on}}^{-}+\frac{l \epsilon}{\left.k^{+}+P_{\pi}^{+}\right)}\right.} \\
\mathcal{T}_{3}^{\prime \mu}=\frac{\operatorname{Tr}\left[\left[\left(k+\not P_{\pi}\right)_{\mathrm{on}}+m\right] \gamma^{5}\left[(k-\not)_{\mathrm{on}}+m\right] \Gamma^{\mu}(k, q) \gamma^{+} \gamma^{5}\right]}{2\left(k^{-}-q^{-}-(k-q)_{\mathrm{on}}^{-}+\frac{l \epsilon}{k^{+}-q^{+}}\right)\left(k^{-}+P_{\pi}^{-}-\left(k+P_{\pi}\right)_{\mathrm{on}}^{-}+\frac{l \epsilon}{k^{+}+P_{\pi}^{+}}\right)} .
\end{gathered}
$$

In Eq. (D24) the quark propagators generate three poles:

$$
k_{(1)}^{-}=k_{\mathrm{on}}^{-}-\frac{l \epsilon}{k^{+}}, \quad k_{(2)}^{-}=q^{-}+(k-q)_{\mathrm{on}}^{-}-\frac{l \epsilon}{k^{+}-q^{+}}, \quad k_{(4)}^{-}=-P_{\pi}^{-}+\left(k+P_{\pi}\right)_{\mathrm{on}}^{-}-\frac{l \epsilon}{k^{+}+P_{\pi}^{+}},
$$

where

$$
\left(k+P_{\pi}\right)_{\text {on }}^{-}=\frac{\left(\mathbf{k}+\mathbf{P}_{\pi}\right)_{\perp}^{2}+m^{2}}{k^{+}+P_{\pi}^{+}}
$$

Let us assume $q^{+} \geq 0$. Therefore, if $k^{+}>q^{+}$and within the hypotheses stated at the beginning of this Section, there are no poles in the upper complex semiplane and a vanishing result is obtained by closing the $k^{-}$integration contour in this semiplane. Furthermore, if $k^{+}<-P_{\pi}^{+}$, there are no poles in the lower complex semiplane of $k^{-}$. Therefore, one obtains a vanishing result by closing the contour of integration in the lower semiplane. Then, the integral has contributions only for $k^{+}$in the range $-P_{\pi}^{+}<k^{+}<q^{+}$. The integration range can be decomposed in two intervals, $-P_{\pi}^{+} \leq k^{+} \leq 0$ and $0<k^{+} \leq q^{+}$. In the first one, if the integration contour is closed in the lower semiplane, only the pole $k_{(4)}^{-}$falls within the integration contour, while in the second one, if the integration contour is closed in the upper semiplane, only the pole $k_{(2)}^{-}$falls within the integration contour. Then in the range $-P_{\pi}^{+} \leq k^{+} \leq 0$ one has contributions from $\mathcal{J}_{\text {on }}^{\prime \mu}, \mathcal{J}_{2}^{\prime \mu}$ and $\mathcal{J}_{3}^{\prime \mu}$, while in the range $0<k^{+} \leq q^{+}$one has contributions from $\mathcal{J}_{\text {on }}^{\prime \mu}, \mathcal{J}_{1}^{\prime \mu}$ and $\mathcal{J}_{3}^{\prime \mu}$.

As a consequence, $j^{\mu}$ can be decomposed as follows

$$
j^{\mu}=j^{(\mathrm{I}) \mu}+j^{(\mathrm{II}) \mu},
$$

where $j^{(\mathrm{I}) \mu}$ has the integration on $k^{+}$constrained by $-P_{\pi}^{+} \leq k^{+} \leq 0$, while $j^{(\mathrm{II}) \mu}$ has the integration on $k^{+}$in the interval $0<k^{+}<q^{+}$. The valence component of the pion contributes to $j^{(\mathrm{I}) \mu}$ only, while $j^{(\mathrm{II}) \mu}$ is the contribution of the pair-production mechanism from an incoming virtual photon with $q^{+}>0 \quad[14,19,21,22,26,27,41]$. Performing the $k^{-}$integration, the two contributions to $j^{\mu}$ are given by the following expressions:

$$
j^{(\mathrm{I}) \mu}=\frac{e}{(2 \pi)^{3}} \frac{m^{2}}{f_{\pi}^{2}} N_{c} \int_{-P_{\pi}^{+}}^{0} \frac{d k^{+} d \mathbf{k}_{\perp}}{\left(k^{+}+P_{\pi}^{+}\right) k^{+}\left(q^{+}-k^{+}\right)}\left[T_{\mathrm{on},(4)}^{\prime \mu}+T_{2,(4)}^{\prime \mu}+T_{3,(4)}^{\prime \mu}\right]\left[\bar{\Lambda}_{\pi^{\prime}}\left(k+P_{\pi}, P_{\pi^{\prime}}\right) \Lambda_{\pi}\left(-k, P_{\pi}\right)\right]_{k^{-}=-P_{\pi}^{-}+\left(k+P_{\pi}\right)_{\mathrm{on}}^{-}}
$$

$$
j^{(\mathrm{II}) \mu}=-\frac{e}{(2 \pi)^{3}} \frac{m^{2}}{f_{\pi}^{2}} N_{c} \int_{0}^{q^{+}} \frac{d k^{+} d \mathbf{k}_{\perp}}{\left(k^{+}+P_{\pi}^{+}\right) k^{+}\left(q^{+}-k^{+}\right)}\left[T_{\mathrm{on},(2)}^{\prime \mu}+T_{1,(2)}^{\prime \mu}+T_{3,(2)}^{\prime \mu}\right]\left[\bar{\Lambda}_{\pi^{\prime}}\left(k+P_{\pi}, P_{\pi^{\prime}}\right) \Lambda_{\pi}\left(-k, P_{\pi}\right)\right]_{k^{-}=q^{-}+(k-q)_{\mathrm{on}}^{-}},
$$

where

$$
T_{\text {on, (4) }}^{\prime \mu}=\frac{\operatorname{Tr}\left[\left[\left(k+\not P_{\pi}\right)_{\text {on }}+m\right] \gamma^{5}\left[(k-\not)_{\text {on }}+m\right] \Gamma^{\mu}(4)\left(k_{\text {on }}+m\right) \gamma^{5}\right]}{\left[P_{\pi}^{-}-\left(k+P_{\pi}\right)_{\text {on }}^{-}+k_{\text {on }}^{-}\right]\left[P_{\pi^{\prime}}^{-}-\left(k+P_{\pi}\right)_{\text {on }}^{-}+(k-q)_{\text {on }}^{-}\right]}
$$




$$
\begin{aligned}
& T_{\mathrm{on},(2)}^{\prime \mu}=\frac{\operatorname{Tr}\left[\left[\left(k+\not P_{\pi}\right)_{\mathrm{on}}+m\right] \gamma^{5}\left[(k-\not)_{\mathrm{on}}+m\right] \Gamma^{\mu}(2)\left(k_{\mathrm{on}}+m\right) \gamma^{5}\right]}{\left[q^{-}+(k-q)_{\mathrm{on}}^{-}-k_{\mathrm{on}}^{-}+\imath \epsilon\right]\left[P_{\pi^{\prime}}^{-}-\left(k+P_{\pi}\right)_{\mathrm{on}}^{-}+(k-q)_{\mathrm{on}}^{-}\right]} \\
& T_{1,(2)}^{\prime \mu}=\frac{\operatorname{Tr}\left[\gamma^{+} \gamma^{5}\left[(k-\not q)_{\mathrm{on}}+m\right] \Gamma^{\mu}(2)\left(k_{\mathrm{on}}+m\right) \gamma^{5}\right]}{2\left[q^{-}+(k-q)_{\mathrm{on}}^{-}-k_{\mathrm{on}}^{-}+\imath \epsilon\right]} \\
& T_{2,(4)}^{\prime \mu}=-\frac{\operatorname{Tr}\left[\left[\left(\not k+\not P_{\pi}\right)_{\mathrm{on}}+m\right] \gamma^{5} \gamma^{+} \Gamma^{\mu}(4)\left(k_{\mathrm{on}}+m\right) \gamma^{5}\right]}{2\left[P_{\pi}^{-}-\left(k+P_{\pi}\right)_{\mathrm{on}}^{-}+k_{\mathrm{on}}^{-}\right]} \\
& T_{3,(4)}^{\prime \mu}=-\frac{\operatorname{Tr}\left[\left[\left(\not k+\not P_{\pi}\right)_{\text {on }}+m\right] \gamma^{5}\left[(\not k-\not)_{\text {on }}+m\right] \Gamma^{\mu}(4) \gamma^{+} \gamma^{5}\right]}{2\left[P_{\pi^{\prime}}^{-}-\left(k+P_{\pi}\right)_{\mathrm{on}}^{-}+(k-q)_{\mathrm{on}}^{-}\right]} \\
& T_{3,(2)}^{\prime \mu}=\frac{\operatorname{Tr}\left[\left[\left(k+\not P_{\pi}\right)_{\mathrm{on}}+m\right] \gamma^{5}\left[(k-\not k)_{\mathrm{on}}+m\right] \Gamma^{\mu}(2) \gamma^{+} \gamma^{5}\right]}{2\left[P_{\pi^{\prime}}^{-}-\left(k+P_{\pi}\right)_{\mathrm{on}}^{-}+(k-q)_{\mathrm{on}}^{-}\right]}
\end{aligned}
$$

with

$$
\Gamma^{\mu}(4)=\Gamma^{\mu}\left(k^{+}, \mathbf{k}_{\perp}, k^{-}=k_{(4)}^{-}, q\right) .
$$

The contributions $j^{(\mathrm{I}) \mu}$ and $j^{(\mathrm{II}) \mu}$ are represented by diagrams (a) and (b) of Fig. 3, respectively.

\section{a. Valence region contribution}

Let us change integration variables in Eq. (D36) for the valence contribution, defining $k^{\prime+}=k^{+}+P_{\pi}^{+}$and $\mathbf{k}_{\perp}^{\prime}=$ $\mathbf{k}_{\perp}+\mathbf{P}_{\pi \perp}$, with $\left(k^{\prime+}, \mathbf{k}_{\perp}^{\prime}\right)$ the light-front momentum of a quark in the valence range. Then $j^{(\mathrm{I}) \mu}$ acquaints the following more familiar expression

$$
j^{(\mathrm{I}) \mu}=\frac{e}{(2 \pi)^{3}} \frac{m^{2}}{f_{\pi}^{2}} N_{c} \int_{0}^{P_{\pi}^{+}} \frac{d k^{\prime+} d \mathbf{k}_{\perp}^{\prime}}{\left(k^{\prime+}-P_{\pi}^{+}\right) k^{\prime+}\left(P_{\pi^{\prime}}^{+}-k^{\prime+}\right)}\left[T_{\mathrm{on},(4)}^{\prime \mu}+T_{2,(4)}^{\prime \mu}+T_{3,(4)}^{\prime \mu}\right]\left[\bar{\Lambda}_{\pi^{\prime}}\left(k^{\prime}, P_{\pi^{\prime}}\right) \Lambda_{\pi}\left(P_{\pi}-k^{\prime}, P_{\pi}\right)\right]_{k^{\prime}=k_{\mathrm{on}}^{\prime}},
$$

where

$$
k_{\mathrm{on}}^{\prime-}=\frac{\left(\mathbf{k}_{\perp}^{\prime 2}+m^{2}\right)}{k^{\prime+}}
$$

and we have defined $k^{-}+P_{\pi}^{-}=k^{\prime-}$. The quantities $T_{\mathrm{on},(4)}^{\prime \mu}, T_{2,(4)}^{\prime \mu}, T_{3,(4)}^{\prime \mu}$ can now be expressed as follows:

$$
\begin{gathered}
T_{\mathrm{on},(4)}^{\prime \mu}=\left[P_{\pi^{\prime}}^{+} P_{\pi}^{+}\right] \frac{\operatorname{Tr}\left[\left(k_{\mathrm{on}}^{\prime}+m\right) \gamma^{5}\left[\left(k^{\prime}-\not P_{\pi^{\prime}}\right)_{\mathrm{on}}+m\right] \Gamma^{\mu}(4)\left[\left(k^{\prime}-\not P_{\pi}\right)_{\mathrm{on}}+m\right] \gamma^{5}\right]}{\left[m_{\pi^{\prime}}^{2}-M_{0}^{2}\left(k^{\prime+}, \mathbf{k}_{\perp}^{\prime} ; P_{\pi^{\prime}}^{+}, \mathbf{P}_{\pi^{\prime} \perp}\right)\right]\left[m_{\pi}^{2}-M_{0}^{2}\left(k^{\prime+}, \mathbf{k}_{\perp}^{\prime} ; P_{\pi}^{+}, \mathbf{P}_{\pi \perp}\right)\right]} \\
T_{2,(4)}^{\prime \mu}=-P_{\pi}^{+} \frac{\operatorname{Tr}\left[\left(k_{\mathrm{on}}^{\prime \prime}+m\right) \gamma^{5} \gamma^{+} \Gamma^{\mu}(4)\left[\left(k^{\prime}-\not P_{\pi}\right)_{\mathrm{on}}+m\right] \gamma^{5}\right]}{2\left[m_{\pi}^{2}-M_{0}^{2}\left(k^{\prime+}, \mathbf{k}_{\perp}^{\prime} ; P_{\pi}^{+}, \mathbf{P}_{\pi \perp}\right)\right]} \\
T_{3,(4)}^{\prime \mu}=-P_{\pi \prime}^{+} \frac{\operatorname{Tr}\left[\left(\not k_{\mathrm{on}}^{\prime \prime}+m\right) \gamma^{5}\left[\left(\not k^{\prime \prime}-\not \not_{\pi^{\prime}}\right)_{\mathrm{on}}+m\right] \Gamma^{\mu}(4) \gamma^{+} \gamma^{5}\right]}{2\left[m_{\pi^{\prime}}^{2}-M_{0}^{2}\left(k^{\prime+}, \mathbf{k}_{\perp}^{\prime} ; P_{\pi^{\prime}}^{+} \mathbf{P}_{\pi^{\prime} \perp}\right)\right]} .
\end{gathered}
$$

In Eq. (D45) both the vertex functions have the quark momentum fractions $k^{\prime+} / P_{\pi^{\prime}}^{+}$and $\left(P_{\pi}-k^{\prime}\right)^{+} / P_{\pi}^{+}$in the valencesector range [0,1]. Note that the on-shell momenta in Eq. (D47) allow one to retrieve the relativistic spin coupling factors with the spin $1 / 2$ Melosh rotations automatically included $[8,9,50]$.

\section{b. Pair-production contribution}

By making use of Eq. (D13) the quantities $T_{\mathrm{on},(2)}^{\prime \mu}, T_{1,(2)}^{\prime \mu}, T_{3,(2)}^{\prime \mu}$ in the pair-production contribution [Eq. (23)] become 


$$
\begin{gathered}
T_{\mathrm{on},(2)}^{\prime \mu}=P_{\pi^{\prime}}^{+} \frac{\operatorname{Tr}\left[\left[\left(k+\not P_{\pi}\right)_{\mathrm{on}}+m\right] \gamma^{5}\left[(k-\not)_{\mathrm{on}}+m\right] \Gamma^{\mu}(2)\left(k_{\mathrm{on}}+m\right) \gamma^{5}\right]}{\left[q^{-}-q_{0}^{-}+\imath \epsilon\right]\left[m_{\pi^{\prime}}^{2}-M_{0}^{2}\left(k^{\prime+}, \mathbf{k}_{\perp}^{\prime} ; P_{\pi^{\prime}}^{+}, \mathbf{P}_{\pi^{\prime} \perp}\right)\right]}, \\
T_{1,(2)}^{\prime \mu}=\frac{\operatorname{Tr}\left[\gamma^{+} \gamma^{5}\left[(k-\not)_{\mathrm{on}}+m\right] \Gamma^{\mu}(2)\left(k_{\mathrm{on}}+m\right) \gamma^{5}\right]}{2\left[q^{-}-q_{0}^{-}+\imath \epsilon\right]}, \\
T_{3,(2)}^{\prime \mu}=P_{\pi^{\prime}}^{+} \frac{\operatorname{Tr}\left[\left[\left(k+\not P_{\pi}\right)_{\mathrm{on}}+m\right] \gamma^{5}\left[(k-\not)_{\mathrm{on}}+m\right] \Gamma^{\mu}(2) \gamma^{+} \gamma^{5}\right]}{2\left[m_{\pi^{\prime}}^{2}-M_{0}^{2}\left(k^{\prime+}, \mathbf{k}_{\perp}^{\prime} ; P_{\pi^{\prime}}^{+}, \mathbf{P}_{\pi^{\prime} \perp}\right)\right]}
\end{gathered}
$$

where $q_{0}^{-}=k_{\text {on }}^{-}+(q-k)_{\text {on }}^{-}$and $k^{\prime+}=k^{+}+P_{\pi}^{+}, \mathbf{k}_{\perp}^{\prime}=$ $\mathbf{k}_{\perp}+\mathbf{P}_{\pi \perp}$.

\section{APPENDIX E: NORMALIZATION OF THE VECTOR-MESON WAVE FUNCTION}

The light-front wave function of vector mesons includes the relativistic spin part, as we have written in Eq. (31), so one has to consider the whole structure of the wave function to obtain the normalization of the valence component of the state. We normalize the wave function using the good component of the vector current, imposing that

$$
\left\langle\phi_{n, \lambda}\left|j^{+}(0)\right| \phi_{n, \lambda}\right\rangle=2 M_{n} P_{q \bar{q}, n},
$$

where it appears the probability of the valence component in the vector meson, $P_{q \bar{q} ; n}$, which is estimated in the next Appendix E.

The matrix element of the good component of the current in impulse approximation is represented by a Feynman triangle diagram. After the integration over the light-front energy, performed disregarding the singularities of the VM vertex in the $k^{-}$complex-plane, one obtains the contribution of the valence wave function to the normalization:

$$
\begin{aligned}
P_{q \bar{q}, n}= & \frac{1}{2 M_{n}} \frac{N_{c}}{16 \pi^{3}} \int_{0}^{M_{n}} \frac{d k^{+}}{\left(k^{+}\right)^{2}\left(M_{n}-k^{+}\right)} \\
& \times \int d \mathbf{k}_{\perp} \mathcal{N}\left(k^{+}, \mathbf{k}_{\perp}\right)\left|\psi_{n}\left(k^{+}, \mathbf{k}_{\perp}, M_{n}, \mathbf{0}_{\perp}\right)\right|^{2} .
\end{aligned}
$$

In Eq. (E2) the quantity $\mathcal{N}\left(k^{+}, \mathbf{k}_{\perp}\right)$ is the following trace

$$
\begin{aligned}
\mathcal{N}\left(k^{+}, \mathbf{k}_{\perp}\right)= & \operatorname{Tr}\left[\left[\left(\not k-\not P_{n}\right)_{\text {on }}+m\right]\right. \\
& \times\left[\boldsymbol{\epsilon}_{\lambda}+\left(k_{\mathrm{on}}-\left(P_{n}-k\right)_{\mathrm{on}}\right) \cdot \epsilon_{\lambda} H_{S}\left(M_{0}\right)\right] \\
& \times\left(k_{\mathrm{on}}+m\right) \gamma^{+}\left(\not_{\mathrm{on}}+m\right) \\
& \left.\times\left[\boldsymbol{\epsilon}_{\lambda}+\left(k_{\mathrm{on}}-\left(P_{n}-k\right)_{\mathrm{on}}\right) \cdot \boldsymbol{\epsilon}_{\lambda} H_{S}\left(M_{0}\right)\right]\right]
\end{aligned}
$$

where $H_{S}\left(M_{0}^{2}\right)$ is defined in Eq. (100).

To evaluate the normalization, we choose the polarization in the transverse direction, which is free of the pair term contribution in the limit of zero momentum transfer $[27,49]$.

\section{APPENDIX F: ESTIMATE OF THE PROBABILITY OF THE VALENCE COMPONENT}

In this Appendix we construct in the Fock space of constituent $q \bar{q}$ pairs a schematic model for the light-front square mass operator $\hat{M}^{2}$ which allows one to roughly estimate the probability of the valence component in the $n$th excited vector-meson state, $P_{q \bar{q} ; n}$. We are looking for a square mass operator in the Fock space with a spectrum where the mass of the $n$th vector meson grows with $\sqrt{n}$, as occurs experimentally $[36,37]$.

Let us denote by $i \geq 0$ the number of $q \bar{q}$ pairs and by $|i\rangle_{0}$ the noninteracting Fock state with $i$ pairs.

Let us suppose that the free mass operator $\hat{M}_{0}$ is additive in the number of pairs and therefore that the noninteracting Fock-state $|i\rangle_{0}$ is eigenfunction of the free squared mass operator with eigenvalue $\alpha^{2} i^{2}$ :

$$
\hat{M}_{0}^{2}|i\rangle_{0}=\alpha^{2} i^{2}|i\rangle_{0}
$$

where $\alpha$ is the energy of a free $q \bar{q}$ state.

We suppose that the interaction $M_{I}^{2}$ in the squared massoperator $\left(\hat{M}^{2}=\hat{M}_{0}^{2}+\hat{M}_{I}^{2}\right)$ :

(i) has constant diagonal matrix elements

$$
{ }_{0}\left\langle i\left|\hat{M}_{I}^{2}\right| i\right\rangle_{0}=\frac{2}{\delta^{2}}-c
$$

(ii) mixes the state $|i\rangle_{0}$ with the states $|(i-1)\rangle_{0}$ and $|(i+1)\rangle_{0}$, and is attractive and constant:

$$
{ }_{0}\left\langle(i+1)\left|\hat{M}_{I}^{2}\right| i\right\rangle_{0}={ }_{0}\left\langle(i-1)\left|\hat{M}_{I}^{2}\right| i\right\rangle_{0}=-\frac{1}{\delta^{2}},
$$

while the other matrix elements of $\hat{M}_{I}^{2}$ are supposed to be zero.

The eigenvalue equation for the squared mass operator is

$$
\hat{M}_{0}^{2}|n\rangle+\hat{M}_{I}^{2}|n\rangle=M_{n}^{2}|n\rangle,
$$

where the $n$th excited state of the meson has mass $M_{n}$. The VM wave function for the $n$th excited state in the Fock space is given by 


$$
|n\rangle=\sum_{i \geq 1} a_{n, i}|i\rangle_{0}
$$

and the amplitudes $a_{n, i}$ are normalized as follows:

$$
\sum_{i \geq 1}\left|a_{n, i}\right|^{2}=1 .
$$

In the above sums one has $i \geq 1$, since the vector mesons have quantum numbers different from the vacuum and then $a_{n, 0}=0$.

Introducing the interaction defined by Eqs. (F2) and (F3) into Eq. (F4) and projecting the eigenvalue equation in the Fock-space state basis $\left\{|i\rangle_{0}\right\}$, one has

$$
\alpha^{2} i^{2} a_{n, i}-\frac{a_{n, i+1}-2 a_{n, i}+a_{n, i-1}}{\delta^{2}}=\left(M_{n}^{2}+c\right) a_{n, i} .
$$

If we define $x=\Delta \cdot i$, with $\Delta>0$, then Eq. (F7) can be rewritten as follows:

$$
\begin{aligned}
& \frac{\delta^{2} \alpha^{2}}{\Delta^{4}} x^{2} a_{n, i}-\frac{\left[a_{n, i+1}-2 a_{n, i}+a_{n, i-1}\right]}{\Delta^{2}} \\
& \quad=\frac{\delta^{2}}{\Delta^{2}}\left(M_{n}^{2}+c\right) a_{n, i} .
\end{aligned}
$$

Then defining $\alpha \delta / \Delta^{2}=\Omega / 4$ and going to the continuous limit one gets

$$
\frac{\Omega^{2}}{16} x^{2} a_{n}(x)-\frac{d^{2} a_{n}(x)}{d x^{2}}=\lambda_{n} a_{n}(x),
$$

with the boundary condition $a_{n}(0)=0$, in order to reflect the constraint $a_{n, 0}=0$. Trivially, because of this constraint the eigenvalues and the eigenstates of $\hat{M}^{2}$ correspond to the odd eigenvalues and eigenstates of the unidimensional harmonic oscillator, namely

$$
\begin{gathered}
\lambda_{n}=\frac{\Omega}{2}\left(n+\frac{1}{2}\right), \\
a_{n}(x)=\sqrt{\frac{2}{2^{n} n !}}\left[\frac{\Omega}{4 \pi}\right]^{1 / 4} H_{n}(\xi) e^{-\xi^{2} / 2},
\end{gathered}
$$

where $H_{n}(\xi)$ are the Hermite polynomials and

$$
\xi=\sqrt{\frac{\Omega}{4}} x .
$$

The function $a_{n}(x)$ in our case has to be normalized as follow:

$$
\int_{0}^{\infty}\left|a_{n}(x)\right|^{2} d x=1
$$

in order to have the proper correspondence with Eq. (F6). This normalization explains the presence of the factor $\sqrt{2}$ in the definition of $a_{n}(x)$ [Eq. (F11)].

Then, defining $\alpha / \delta=\omega / 4$, from the eigenvalues $\lambda_{n}$ of Eq. (F9) one can obtain the masses of the vector-mesons, viz

$$
M_{n}^{2}=\frac{\omega}{2}\left(n+\frac{1}{2}\right)-c=\omega\left(n_{\mathrm{ex}}+\frac{3}{4}\right)-c,
$$

where only the odd values of $n$, namely $n=2 n_{\mathrm{ex}}+1$, are allowed. The number $n_{\mathrm{ex}}$ is the excitation number $0,1,2,3$ $\ldots$ of the vector mesons, with zero for the meson ground state. Then the square mass of the vector mesons is given by

$$
M_{n_{\mathrm{ex}}}^{2}=\omega \cdot n_{\mathrm{ex}}+M_{\mathrm{g} . \mathrm{s} .}^{2},
$$

where the meson ground state has mass $M_{\text {g.s. }}^{2}=3 \omega / 4-c$ (note that, given $\omega$, the constant $c$ is fixed).

At this point we exactly retrieve the experimental spectrum law [36,37], which is the motivation of this simple model.

The final step is the estimate of the probability of the valence state, namely $P_{q \bar{q}, n}$. From a comparison of the discrete and the continuum case of our model, one can associate to the probability $\left|a_{n, i}\right|^{2}$ of the wave-function component with $i q \bar{q}$ pairs, Eq. (F5) the quantity

$$
\begin{aligned}
\int_{(i-1) \Delta}^{i \Delta} d x\left|a_{n}(x)\right|^{2} & =\frac{2}{2^{n} n !}\left[\frac{\Omega}{4 \pi}\right]^{1 / 2} \int_{(i-1) \Delta}^{i \Delta} d x\left|H_{n}(\xi)\right|^{2} e^{-\xi^{2}} \\
& =\frac{2}{2^{n} n ! \sqrt{\pi}} \int_{(i-1) \delta \sqrt{\omega} / 2}^{i \delta \sqrt{\omega} / 2} d \xi\left|H_{n}(\xi)\right|^{2} e^{-\xi^{2}} .
\end{aligned}
$$

Then the probability $P_{q \bar{q}, n_{\mathrm{ex}}}$ of the valence component is given by the quantity

$$
\begin{aligned}
P_{q \bar{q}, n_{\mathrm{ex}}} & =\left|a_{n, 1}\right|^{2} \sim \int_{0}^{\Delta} d x\left|a_{n}(x)\right|^{2} \\
& =\frac{2}{2^{n} n ! \sqrt{\pi}} \int_{0}^{\delta \sqrt{\omega} / 2} d \xi\left|H_{n}(\xi)\right|^{2} e^{-\xi^{2}} .
\end{aligned}
$$

Imposing that $P_{q \bar{q} ; 0}$, i.e. the valence component probability in the ground state of the vector mesons, is about the same as the one found in constituent quark models of the pion [21], i.e. it is equal to 0.77 , we obtain $\omega^{1 / 2} \delta=2.94$.

Alternatively, let us evaluate the average number of $q \bar{q}$ pairs in the vector meson:

$$
\sqrt{\left\langle i^{2}\right\rangle}=\frac{\sqrt{\left\langle x^{2}\right\rangle}}{\Delta}=\frac{2}{\Omega^{1 / 2} \Delta} \sqrt{n+\frac{1}{2}}=\frac{2}{\omega^{1 / 2} \delta} \sqrt{2 n_{\mathrm{ex}}+\frac{3}{2}} .
$$

Then we can estimate the probability for the lowest Fock component to be roughly given by

$$
P_{q \bar{q} ; n_{\mathrm{ex}}}=\frac{\delta \omega^{1 / 2}}{2 \sqrt{2 n_{\mathrm{ex}}+\frac{3}{2}}} .
$$

If the value $\omega^{1 / 2} \delta=2$ is used in this last estimate, the probabilities considered in Ref. [26] are obtained. With this choice, the valence component probability in the ground state of the vector meson is equal to $\sim 0.8$. 


\section{APPENDIX G: EVALUATION OF $g_{V n}^{+}\left(q^{2}\right)$ IN THE TIMELIKE REGION}

First of all let us evaluate $\sum_{\lambda}$ of Eq. (44). The momentum of the vector meson is $P_{n}^{\mu} \equiv\left\{P_{n}^{-}=\left(\left|\mathbf{q}_{\perp}\right|^{2}+\right.\right.$ $\left.\left.M_{n}^{2}\right) / q^{+}, \mathbf{q}_{\perp}, q^{+}\right\}$and the momentum of the virtual photon is $q^{\mu} \equiv\left\{q^{-}, \mathbf{q}_{\perp}, q^{+}\right\}$(as already noted, see Fig. 4 , at the production vertex the LF three-momentum is conserved). In a frame where $\mathbf{q}_{\perp}=0$, one has $q^{-}=q^{2} / q^{+}$for the photon, while for the vector meson $P_{n}^{-}=M_{n}^{2} / q^{+}$. Using Cartesian components for the four-vectors, i.e. $a^{\mu} \equiv$ $\left[a^{0}, \mathbf{a}\right]$, in this frame the three polarization four-vectors are given by

$$
\begin{gathered}
\epsilon_{x}^{\mu} \equiv[0,1,0,0], \quad \epsilon_{y}^{\mu} \equiv[0,0,1,0], \\
\epsilon_{z}^{\mu} \equiv\left[P_{n z} / M_{n}, 0,0, \sqrt{1+\eta}\right],
\end{gathered}
$$

where $\eta=P_{n z}^{2} / M_{n}^{2}$. Let us recall that, in the frame we are adopting, $P_{n z}=\left(q^{+}-P_{n}^{-}\right) / 2=\left(q^{+2}-M_{n}^{2}\right) / 2 q^{+}$. Therefore, in the reference frame defined by $\mathbf{q}_{\perp}=0$ and $q^{+}>0$ the polarization four-vector $\epsilon_{z}^{\mu}$ does not have a defined sign for the zero component. The plus-component of $\epsilon_{z}^{\mu}$ is given by

$$
\begin{aligned}
\epsilon_{z}^{+} & =\frac{P_{n z}}{M_{n}}+\sqrt{1+\frac{P_{n z}^{2}}{M_{n}^{2}}} \\
& =\frac{q^{+2}-M_{n}^{2}}{2 q^{+} M_{n}}+\sqrt{1+\left(\frac{q^{+2}-M_{n}^{2}}{2 q^{+} M_{n}}\right)^{2}} .
\end{aligned}
$$

The plus component of the other polarization four-vectors are vanishing (i.e., $\epsilon_{x}^{+}=\epsilon_{y}^{+}=0$ ) and therefore we have $\sum_{\lambda}\left[\epsilon_{\lambda}^{+}\left(P_{n}\right)\right]^{*} \epsilon_{\lambda}\left(P_{n}\right) \cdot \hat{V}_{n}=\left[\epsilon_{z}^{+}\left(P_{n}\right)\right]^{*} \epsilon_{z}\left(P_{n}\right) \cdot \hat{V}_{n}$.

Each term of $\sum_{n}$ in Eq. (69) is invariant under LF boosts, that are kinematical, and therefore to simplify the calculations it can be evaluated in the rest frame of the corresponding resonance. In the rest frame of the $n$ th-resonance one has $q^{+}=M_{n}$ and $q^{-}=q^{2} / M_{n}$ for the photon, while $P_{n}^{+}=P_{n}^{-}=M_{n}$ for the vector meson. This means that we choose a different frame for each resonance, but all these frames are related by kinematical LF boosts along the $z$ axis to each other, and to the Breit frame where $q^{+}=$ $-q^{-}=\sqrt{-q^{2}}$, adopted in previous analyses of the SL region (we have always $\mathbf{q}_{\perp}=0$ ) [21,29].

Then, in the evaluation of the sum in Eq. (69) one has $\epsilon_{z}^{+}=1$ and

$$
\sum_{\lambda}\left[\epsilon_{\lambda}^{+}\left(P_{n}\right)\right]^{*} \epsilon_{\lambda}\left(P_{n}\right) \cdot \hat{V}_{n}=-\hat{V}_{n z}
$$

for the contribution of any resonance. In conclusion we have

$$
\begin{aligned}
g_{V n}^{+}\left(q^{2}\right)= & \frac{1}{P_{\bar{\pi}}^{+}-P_{\pi}^{+}} \frac{N_{c}}{(2 \pi)^{3}} \int_{0}^{q^{+}} \frac{\sqrt{2} d k^{+} d \mathbf{k}_{\perp}}{\left(k^{+}-P_{\pi}^{+}\right) k^{+}\left(q^{+}-k^{+}\right)} \\
& \times\left\{\Theta\left(P_{\pi}^{+}-k^{+}\right) \bar{I}_{1, n}+\Theta\left(k^{+}-P_{\pi}^{+}\right) \bar{I}_{2, n}\right\}, \quad(\mathrm{G} 4)
\end{aligned}
$$

where the quantities $\bar{I}_{1, n}$ and $\bar{I}_{2, n}$ can be obtained from Eqs. (54) and (55) replacing $m / f_{\pi}\left[\Lambda_{\bar{\pi}}\left(k-P_{\pi}, P_{\bar{\pi}}\right)\right]_{k^{-}=k_{\text {on }}^{-}}$ and $m / f_{\pi}\left[\bar{\Lambda}_{\pi}\left(k, P_{\pi}\right)\right]_{k^{-}=q^{-}+(k-q)_{\text {on }}^{-}}$with $\mathcal{D}_{\bar{\pi}}$ and $\overline{\mathcal{D}}_{\pi}$, respectively, [see Eqs. (40) and (39)]:

$$
\begin{aligned}
\bar{I}_{1, n}= & \mathcal{D}_{\bar{\pi}}\left\{\frac{\psi_{n}\left(k^{+}, \mathbf{k}_{\perp} ; P_{n}^{+}, \mathbf{0}\right)\left[M_{n}^{2}-M_{0}^{2}\left(k^{+}, \mathbf{k}_{\perp} ; P_{n}^{+}, \mathbf{0}\right]\right.}{\left[q^{2}-M_{0}^{2}\left(k^{+}, \mathbf{k}_{\perp} ; q^{+}, \mathbf{0}\right)+\imath \boldsymbol{\epsilon}\right]}\right. \\
& \left.\times\left[\mathcal{T}_{\mathrm{on},(1, n)}+\mathcal{T}_{1,(1, n)}\right]+\left[\Lambda_{n}\left(k, P_{n}\right)\right]_{k^{-}=k_{\mathrm{on}}^{-}} \mathcal{T}_{2,(1, n)}\right\} \\
\bar{I}_{2, n}= & \overline{\mathcal{D}}_{\pi}\left\{\frac{\psi_{n}\left(k^{+}, \mathbf{k}_{\perp} ; P_{n}^{+}, \mathbf{0}\right)\left[M_{n}^{2}-M_{0}^{2}\left(k^{+}, \mathbf{k}_{\perp} ; P_{n}^{+}, \mathbf{0}\right)\right]}{\left[q^{2}-M_{0}^{2}\left(k^{+}, \mathbf{k}_{\perp} ; q^{+}, \mathbf{0}\right)+\imath \boldsymbol{\epsilon}\right]}\right. \\
& \times\left[\mathcal{T}_{\mathrm{on},(2, n)}+\mathcal{T}_{1,(2, n)}\right] \\
& \left.+\left[\Lambda_{n}\left(k, P_{n}\right)\right]_{k^{-}=q^{-}+(k-q)_{\mathrm{on}}^{-}} \mathcal{T}_{3,(2, n)}\right\}
\end{aligned}
$$

with

$$
\begin{aligned}
\mathcal{T}_{\text {on },(1, n)}= & -\psi_{\pi}^{*}\left(k^{+}, \mathbf{k}_{\perp} ; P_{\pi}^{+}, \mathbf{P}_{\pi \perp}\right) \operatorname{Tr}\left[\left[\left(k-\not P_{\pi}\right)_{\mathrm{on}}+m\right]\right. \\
& \times \gamma^{5}\left[(k-\not)_{\mathrm{on}}+m\right] \\
& \left.\times\left[\hat{V}_{n z}\left(k, k-P_{n}\right)\right]_{\mathrm{on}}\left(k_{\mathrm{on}}+m\right) \gamma^{5}\right]
\end{aligned}
$$

$$
\mathcal{T}_{\text {on, }(2, n)}=\psi_{\bar{\pi}}^{*}\left(\left(k^{+}-P_{\pi}^{+}\right),\left(\mathbf{k}-\mathbf{P}_{\pi}\right)_{\perp} ; P_{\bar{\pi}}^{+}, \mathbf{P}_{\bar{\pi} \perp}\right) \operatorname{Tr}\left[\left[\left(\not k-\not P_{\pi}\right)_{\text {on }}+m\right] \gamma^{5}\left[(\not k-\not q)_{\text {on }}+m\right]\left[\hat{V}_{n z}\left(k, k-P_{n}\right)\right]_{\text {on }}\left(k_{\text {on }}+m\right) \gamma^{5}\right]
$$

$$
\mathcal{T}_{1,(1, n)}=\frac{1}{2} \frac{m}{f_{\pi}}\left[\bar{\Lambda}_{\pi}\left(k ; P_{\pi}\right)\right]_{k^{-}=k_{\mathrm{on}}^{-}} \operatorname{Tr}\left[\gamma^{+} \gamma^{5}\left[(k-\not)_{\mathrm{on}}+m\right]\left[\hat{V}_{n z}\left(k, k-P_{n}\right)\right]_{\mathrm{on}}\left(k_{\mathrm{on}}+m\right) \gamma^{5}\right]
$$

$$
\mathcal{T}_{1,(2, n)}=\frac{1}{2} \frac{m}{f_{\pi}}\left[\Lambda_{\bar{\pi}}\left(k-P_{\pi}, P_{\bar{\pi}}\right)\right]_{k^{-}=q^{-}+(k-q)_{\text {on }}^{-}} \operatorname{Tr}\left[\gamma^{+} \gamma^{5}\left[(k-\not)_{\text {on }}+m\right]\left[\hat{V}_{n z}\left(k, k-P_{n}\right)\right]_{\text {on }}\left(k_{\text {on }}+m\right) \gamma^{5}\right]
$$




$$
\begin{gathered}
\mathcal{T}_{2,(1, n)}=\frac{1}{2} \psi_{\pi}^{*}\left(k^{+}, \mathbf{k}_{\perp} ; P_{\pi}^{+}, \mathbf{P}_{\pi \perp}\right) \operatorname{Tr}\left[\left[\left(k-\not P_{\pi}\right)_{\mathrm{on}}+m\right] \gamma^{5} \gamma^{+}\left[\hat{V}_{n z}\left(k, k-P_{n}\right)\right]_{\mathrm{on}}\left(k_{\mathrm{on}}+m\right) \gamma^{5}\right] \\
\mathcal{T}_{3,(2, n)}=\frac{1}{2} \psi_{\bar{\pi}}^{*}\left(\left(k^{+}-P_{\pi}^{+}\right),\left(\mathbf{k}-\mathbf{P}_{\pi}\right)_{\perp} ; P_{\bar{\pi}}^{+}, \mathbf{P}_{\bar{\pi} \perp}\right) \operatorname{Tr}\left[\left[\left(k-\not P_{\pi}\right)_{\mathrm{on}}+m\right] \gamma^{5}\left[(k-\not)_{\mathrm{on}}+m\right]\left[\hat{V}_{n z}\left(k, k-P_{n}\right)\right]_{\mathrm{on}} \gamma^{+} \gamma^{5}\right]
\end{gathered}
$$

The ${ }^{3} S_{1}$ vector-meson vertex $\left[\hat{V}_{n}\left(k, k-P_{n}\right)\right]_{\text {on }}$ given by Eq. (16), as it was used in previous calculations [8], is completely determined by the kinematical momenta of the individual quark and antiquark. In the ${ }^{3} S_{1}$ vector-meson intrinsic frame, where $q^{+}=M_{n}$ and $\mathbf{q}_{\perp}=\mathbf{0}$, one has

$$
\left[\hat{V}_{n z}\left(k, k-P_{n}\right)\right]_{\mathrm{on}}=\left(\gamma^{3}-\frac{k_{\mathrm{on}}^{3}-\left(P_{n}^{3}-k^{3}\right)_{\mathrm{on}}}{M_{0 n}+2 m}\right),
$$

where $\quad M_{0 n}^{2}=\left(\left|\mathbf{k}_{\perp}\right|^{2}+m^{2}\right) / x(1-x) \quad\left(x=k^{+} / q^{+}=\right.$ $\left.k^{+} / M_{n}\right)$.

The kinematics for the final two-pion state in the particular frame where the photon has momentum $\mathbf{q}_{\perp}=0$, $q^{+}=M_{n}$ and $q^{-}=q^{2} / M_{n}$ can be derived from the energy-momentum conservation, which yields

$$
q^{+}=M_{n}=P_{\bar{\pi}}^{+}+P_{\pi}^{+}, \quad \mathbf{P}_{\bar{\pi} \perp}=-\mathbf{P}_{\pi \perp}
$$

and thus

$$
\begin{aligned}
q^{-} & =P_{\overline{\bar{\pi}}}^{-}+P_{\pi}^{-}=\frac{\left|\mathbf{P}_{\pi \perp}\right|^{2}+m_{\pi}^{2}}{P_{\bar{\pi}}^{+}}+\frac{\left|\mathbf{P}_{\pi \perp}\right|^{2}+m_{\pi}^{2}}{P_{\pi}^{+}} \\
& =\frac{1}{q^{+}} \frac{\left|\mathbf{P}_{\pi \perp}\right|^{2}+m_{\pi}^{2}}{x_{\pi}\left(1-x_{\pi}\right)}
\end{aligned}
$$

where $\quad x_{\pi}=P_{\pi}^{+} / q^{+} \quad$ and $\quad x_{\bar{\pi}}=P_{\bar{\pi}}^{+} / q^{+}=1-x_{\pi}$. Equations (G14) and (G15) put in evidence the relation between the kinematical variables of the virtual photon and the ones of both pion and antipion. In the timelike region the value of $q^{2}$ does not fully determine the values for the four-momenta of the pion and the antipion in the final state of the $\pi \bar{\pi}$ pair. In order to reduce the freedom we make the purely longitudinal choice, i.e. $\mathbf{P}_{\bar{\pi} \perp}=-\mathbf{P}_{\pi \perp}=\mathbf{0}$. Then, from Eqs. (G14) and (G15), one obtains

$$
x_{\pi}=\frac{1}{2} \pm \sqrt{\frac{1}{4}-\frac{m_{\pi}^{2}}{q^{2}}} .
$$

Let us note that the minimum allowed value for $q^{2}$ is $4 m_{\pi}^{2}$. At this threshold value one has $x_{\pi}=1 / 2$. Therefore, since

$$
P_{\bar{\pi}}^{+}-P_{\pi}^{+}=q^{+}-2 P_{\pi}^{+}=q^{+}\left(1-2 x_{\pi}\right)=M_{n}\left(1-2 x_{\pi}\right),
$$

one cannot evaluate Eq. (G4) exactly at threshold, unless an exact cancellation occurs between vanishing numerator and denominator of Eq. (G4). For finite values of $m_{\pi}$, the values $x_{\pi}=1$ or $x_{\pi}=0$ are possible only for an infinite value of the momentum transfer and imply an infinite value of $P_{\pi z}$ or $P_{\bar{\pi} z}$, respectively.

In the limit of a vanishing pion mass $\left(m_{\pi}=0\right)$, Eq. (G16) gives $x_{\pi}=1$ or 0 , which implies that one of the terms of Eq. (G4) vanishes due to the $\Theta$ function. To simplify our calculations, we make the approximation $m_{\pi}=0$ and adopt the choice $x_{\pi}=0$, which implies $P_{\pi}^{+}=$ $0, P_{\pi}^{-}=q^{-}=q^{2} / M_{n}, \quad P_{\bar{\pi}}^{+}=q^{+}=M_{n}$, and $P_{\bar{\pi}}^{-}=0$. Then only the second term of Eq. (G4), containing the quantity $\bar{I}_{2, n}$, gives a contribution to the TL pion form factor.

Furthermore, for $m_{\pi}=0$ one has $\mathcal{T}_{\text {on, }(2, n)}=0$. Indeed, in this limit $\left(\not k-\not p_{\pi}\right)_{\text {on }}=\not k_{\text {on }}$ and

$$
\begin{aligned}
\left(k_{\mathrm{on}}+m\right) \gamma^{5}\left(k_{\mathrm{on}}+m\right) & =\left(k_{\mathrm{on}}+m\right)\left(-k_{\mathrm{on}}+m\right) \gamma^{5} \\
& =\left(k_{\mathrm{on}} k_{\mathrm{on}}+m^{2}\right) \gamma^{5}=0
\end{aligned}
$$

Therefore only the instantaneous contributions $\mathcal{T}_{1,(2, n)}$ and $\mathcal{T}_{3,(2, n)}$ survive in the limit of a vanishing pion mass and can be written as follows:

$$
\begin{aligned}
\mathcal{T}_{1,(2, n)}= & -\frac{1}{2} \frac{m}{f_{\pi}}\left[\Lambda_{\bar{\pi}}\left(k-P_{\pi}, P_{\bar{\pi}}\right)\right]_{k^{-}=q^{-}+(k-q)_{\mathrm{on}}^{-}} \\
& \times \operatorname{Tr}\left[\gamma^{+}\left[(k-\not)_{\mathrm{on}}+m\right]\left[\hat{V}_{n z}\left(k, k-P_{n}\right)\right]_{\mathrm{on}}\right. \\
& \left.\times\left(k_{\mathrm{on}}+m\right)\right]
\end{aligned}
$$

$$
\begin{aligned}
\mathcal{T}_{3,(2, n)}= & \frac{1}{2} \psi_{\bar{\pi}}^{*}\left(k^{+}, \mathbf{k}_{\perp} ; M_{n}, \mathbf{0}_{\perp}\right) \\
& \times \operatorname{Tr}\left[\left[-k_{\mathrm{on}}+m\right]\left[(k-\not)_{\mathrm{on}}+m\right]\right. \\
& \left.\times\left[\hat{V}_{n z}\left(k, k-P_{n}\right)\right]_{\mathrm{on}} \gamma^{+}\right] .
\end{aligned}
$$


[1] P. A. M. Dirac, Rev. Mod. Phys. 21, 392 (1949).

[2] S. J. Brodsky, H. C. Pauli, and S. S. Pinsky, Phys. Rep. 301, 299 (1998).

[3] J. Carbonell, B. Deplanques, V. A. Karmanov, and J.-F. Mathiot, Phys. Rep. 300, 215 (1998).

[4] B. D. Keister and W. N. Polyzou, Adv. Nucl. Phys. 20, 225 (1991).

[5] F. M. Lev, Riv. Nuovo Cimento 16, 1 (1993).

[6] H. J. Weber, Ann. Phys. (N.Y.) 177, 38 (1987); W. Konen and H. J. Weber, Phys. Rev. D 41, 2201 (1990).

[7] P. L. Chung, F. Coester, and W. N. Polyzou, Phys. Lett. B 205, 545 (1988); P. L. Chung and F. Coester, Phys. Rev. D 44, 229 (1991).

[8] W. Jaus, Phys. Rev. D 41, 3394 (1990).

[9] T. Frederico and G. A. Miller, Phys. Rev. D 45, 4207 (1992); 50, 210 (1994).

[10] F. Cardarelli et al., Phys. Lett. B 332, 1 (1994).

[11] F. Cardarelli et al., Phys. Rev. D 53, 6682 (1996).

[12] F. Cardarelli, E. Pace, G. Salmè, and S. Simula, Phys. Lett. B 357, 267 (1995); 371, 7 (1996); 397, 13 (1997); FewBody Syst., Suppl. 8345 (1995); Nucl. Phys. A623, 361c (1997); A666 \& A667, 33c (2000).

[13] F. Cardarelli and S. Simula, Phys. Lett. B 467, 1 (1999); Phys. Rev. C 62, 065201 (2000).

[14] H. W. L. Naus, J.P.C. de Melo, and T. Frederico, FewBody Syst. 24, 99 (1998); J. P. C. de Melo, H. W. L. Naus, and T. Frederico, Phys. Rev. C 59, 2278 (1999).

[15] W. R. B. de Araújo, E. F. Suisso, T. Frederico, M. Beyer, and H. J. Weber, Phys. Lett. B 478, 86 (2000); E. F. Suisso, W.R.B. de Araújo, T. Frederico, M. Beyer, and H.J. Weber, Nucl. Phys. A694, 351 (2001).

[16] W. Jaus, Phys. Rev. D 44, 2851 (1991); 60, 054026 (1999); 63, 053009 (2001).

[17] H.-M. Choi and C.-R. Ji, Nucl. Phys. A679, 735 (2001).

[18] C.-R. Ji and H.-M. Choi, Phys. Lett. B 513, 330 (2001).

[19] B. L. G. Bakker, H.-M. Choi, and C.-R. Ji, Phys. Rev. D 63, 074014 (2001).

[20] C.-W. Hwang, Phys. Rev. D 64, 034011 (2001).

[21] J.P. B.C. de Melo, T. Frederico, E. Pace, and G. Salmè, Nucl. Phys. A707, 399 (2002); Braz. J. Phys. 33, 301 (2003); Few-Body Syst. 36, 189 (2005).

[22] B. L. G. Bakker, H.-M. Choi, and C.-R. Ji, Phys. Rev. D 65, 116001 (2002).

[23] S. J. Brodsky, Braz. J. Phys. 34, 157 (2004).

[24] L. Martinovic and J.P. Vary, Phys. Rev. D 64, 105016 (2001).

[25] K. Itakura and S. Maedan, Phys. Rev. D 61, 045009 (2000).

[26] J.P. B. C. de Melo, T. Frederico, E. Pace, and G. Salmè, Phys. Lett. B 581, 75 (2004).

[27] J.P. B. C. de Melo, J.H. O. Sales, T. Frederico, and P. U. Sauer, Nucl. Phys. A631, 574c (1998); J. P. B. C. de Melo, T. Frederico, H. W. L. Naus, and P. U. Sauer, Nucl. Phys. A660, 219 (1999).

[28] D. Ashery, Nucl. Phys. B, Proc. Suppl. 108, 321 (2002), and references therein.

[29] F. M. Lev, E. Pace, and G. Salmè, Nucl. Phys. A641, 229 (1998); Few-Body Syst., Suppl. 10, 135 (1998); Phys. Rev. Lett. 83, 5250 (1999); Phys. Rev. C 62, 064004 (2000); Nucl. Phys. A663, 365 (2000); E. Pace and G. Salmè,
Nucl. Phys. A684, 487 (2001); A689, 441 (2001).

[30] S. Mandelstam, Proc. R. Soc. A 233, 248 (1955).

[31] B. L. G. Bakker and C.-R. Ji, Phys. Rev. D 62, 074014 (2000).

[32] H. B. O'Connell et al., Phys. Lett. B 513, 330 (2001).

[33] H. C. Pauli, Eur. Phys. J. C 7, 289 (1999); in New Directions in Quantum Chromodynamics, edited by C. R. Ji and D. P. Min (AIP, Melville, NY, 1999), pp. 80-139; Nucl. Phys. B, Proc. Suppl. 90, 154 (2000).

[34] T. Frederico and H.-C. Pauli, Phys. Rev. D 64, 054007 (2001).

[35] T. Frederico, H.-C. Pauli, and S.-G. Zhou, Phys. Rev. D 66, 054007 (2002); 66, 116011 (2002).

[36] F. Iachello, N. C. Mukhopadhyay, and L. Zhang, Phys. Rev. D 44, 898 (1991).

[37] A. V. Anisovitch, V. V. Anisovich, and A. V. Sarantsev, Phys. Rev. D 62, 051502(R) (2000).

[38] S. Eidelman et al., Phys. Lett. B 592, 1 (2004).

[39] C. Bruch, A. Khodjamirian, and J. H. Kühn, Eur. Phys. J. C 39, 41 (2005).

[40] S. Gardner and H. B. O’Connell, Phys. Rev. D 57, 2716 (1998).

[41] M. Sawicki, Phys. Rev. D 44, 433 (1991); 46, 474 (1992).

[42] B. C. Tiburzi and G. A. Miller, Phys. Rev. D 65, 074009 (2002); 67, 054014 (2003); 67, 054015 (2003).

[43] D. Lurié, A. J. Macfarlane, and Y. Takahashi, Phys. Rev. 140, B1091 (1965).

[44] C. Itzykson and J.B. Zuber, Quantum Field Theory (McGraw-Hill, New York, 1980).

[45] F. Halzen and A. D. Martin, Quarks and Leptons (John Wiley and Sons, New York, 1984), p. 138; L. D. Landau, V. B. Berestetskij, L. Lifshitz, and L. P. Pitaevskij, Teoria Quantistica Relativistica (Editori Riuniti, Edizioni Mir, Mosca, 1978), pp. 359, 589.

[46] C. J. Gounaris and J. J. Sakurai, Phys. Rev. Lett. 21, 244 (1968).

[47] F. Klingl, N. Kaiser, and W. Weise, Z. Phys. A 356, 193 (1996).

[48] M. Benayoun, S. Eidelman, K. Maltman, H. B. O'Connell, B. Shwartz, and A. G. Williams, Eur. Phys. J. C 2, 269 (1998).

[49] J. P. B. C. de Melo and T. Frederico, Phys. Rev. C 55, 2043 (1997).

[50] W. R. B. de Araújo, T. Frederico, M. Beyer, and H. W. Weber, J. Phys. G 25, 1589 (1999).

[51] J. H. O. Sales, T. Frederico, B. V. Carlson, and P. U. Sauer, Phys. Rev. C 61, 044003 (2000); 63, 064003 (2001).

[52] S. Godfrey and Nathan Isgur, Phys. Rev. D 32, 189 (1985).

[53] A. De Rujula, H. Georgi, and S. L. Glashow, Phys. Rev. D 12, 147 (1975).

[54] R. R. Akhmetshin et al., Phys. Lett. B 509, 217 (2001).

[55] A. V. Anisovich et al., Phys. Lett. B 542, 8 (2002).

[56] R. Baldini et al., Eur. Phys. J. C 11, 709 (1999); Nucl. Phys. A666, 38 (2000); (private communication).

[57] J. Volmer et al., Phys. Rev. Lett. 86, 1713 (2001).

[58] G. P. Lepage and S. J. Brodsky, Phys. Rev. D 22, 2157 (1980).

[59] J.P. B. C. de Melo, T. Frederico, E. Pace, and G. Salmè (unpublished). 